\title{
Supporting Information for \\ Removal of Pathogens and Chemicals of Emerging Concern by Pilot-Scale FO-RO Hybrid Units Treating RO Concentrate, Graywater and Sewage for Centralized and Decentralized Potable Reuse
}

\author{
Aleksandra Szczuka ${ }^{1,2}$, Yi-Hsueh Chuang ${ }^{3}$, Felipe C. Chen ${ }^{1}$, Zhong Zhang ${ }^{1,2}$, Erik Desormeaux ${ }^{4}$, \\ Michael Flynn ${ }^{5}$, Jurek Parodi ${ }^{5}$, William A. Mitch ${ }^{1,2, *}$ \\ ${ }^{1}$ Department of Civil and Environmental Engineering, Stanford University, 473 Via Ortega, \\ Stanford, CA, 94305, USA \\ ${ }^{2}$ National Science Foundation Engineering Re-Inventing the Nation's Urban Water \\ Infrastructure (ReNUWIt), USA \\ ${ }^{3}$ Institute of Environmental Engineering, National Chiao Tung University No. 1001, University \\ Road, East District, Hsinchu City, Taiwan 300 \\ ${ }^{4}$ Porifera, Inc., 1575 Alvarado St., San Leandro, CA, 94577, USA \\ ${ }^{5} \mathrm{wH}_{2}$ OSystems, 1289 Reamwood Ave., Sunnyvale, CA, 94089, USA \\ *Corresponding author: email: wamitch@stanford.edu, Phone: 650-725-9298, Fax: 650-723- \\ 7058
}




\section{Contents}

$\begin{array}{ll}\text { Text S1. General water quality analyses. } & \text { S3 }\end{array}$

$\begin{array}{ll}\text { Text S2. } \text { LC-MS/MS methods descriptions. } & \text { S3 }\end{array}$

$\begin{array}{ll}\text { Text S3. DBP methods descriptions. } & \text { S3 }\end{array}$

Text S4. Lab scale FO membrane experiment descriptions. $\quad$ S4

Figure S1. 1,4-Dioxane and chloroform concentrations in sewage and greywater $\quad$ S5

Figure S2. Coliform concentrations in FO-RO effluent for decentralized treatment $\quad$ S6

Figure S3. Total DBP, chloroform and NDMA concentrations in sewage and greywater S7

Figure S4. DBP concentrations throughout a batch run during greywater treatment. $\quad$ S8

Figure S5. DBP concentrations throughout a batch run during sewage treatment. S9

Figure S6. DBP concentrations in the synthetic washwater and greywater blends. $\quad$ S10

Table S1. NSF 350-1 wash water formulation. $\quad$ S11

Table S2. LC-MS/MS gradient method parameters. $\quad$ S11

Table S3. LC-MS/MS detection limits. $\quad$ S12

$\begin{array}{lr}\text { Table S4. Mass spectrometry parameters. } & \text { S12 }\end{array}$

Table S5. Structures and molecular volumes of organic contaminants. S13

Table S6. Chloramine and free chlorine doses for decentralized FO/RO samples. $\quad$ S15

Table S7. LC50 values for halogenated DBPs. $\quad$ S16

Table S8. LECR50 values for $N$-nitrosamines. $\quad$ S17

Table S9. General water quality parameters for treatment of RO concentrate S18 
Table S10. General water quality parameters for treatment of greywater and sewage

Tables S11-S38. DBPs detected in the decentralized reuse application.

Tables S39-S54. DBPs detected in the centralized reuse application.

Text S1. General Water Quality Analyses. Samples collected for basic water quality analysis were analyzed within two hours of collection, all samples apart from those used for total suspended solids (TSS) and chemical oxygen demand (COD) analyses were filtered with a 0.7 micron glass fiber filter (Whatman). TSS (Minimum reporting level (MRL) $=0.1 \mathrm{mg} / \mathrm{L}$ ) were measured using EPA method 160.2. $\mathrm{COD}(\mathrm{MRL}=0.7 \mathrm{mg} \mathrm{COD} / \mathrm{L})$, ammonia $(\mathrm{MRL}=0.015$ $\mathrm{mg}-\mathrm{N} / \mathrm{L})$, nitrate $(\mathrm{MRL}=0.2 \mathrm{mg}-\mathrm{N} / \mathrm{L})$, and nitrite $(\mathrm{MRL}=0.02 \mathrm{mg}-\mathrm{N} / \mathrm{L})$, and total phosphorous $(\mathrm{MRL}=0.1 \mathrm{mg}-\mathrm{P} / \mathrm{L})$ were measured by $\mathrm{HACH}$ methods $8000,10205,8171,8507$, and 8131, respectively. Dissolved organic carbon $(\mathrm{DOC})(\mathrm{MRL}=0.1 \mathrm{mg}-\mathrm{C} / \mathrm{L})$ was analyzed using a Shimadzu TOC-VCSH total organic carbon analyzer. The 5-day biological oxygen demand $(B O D 5)(M R L=0.1 \mathrm{mg} / \mathrm{L})$ was analyzed using EPA method 405.1. Chloride $(\mathrm{MRL}=0.01$ $\mathrm{mg} / \mathrm{L})$, and bromide $(\mathrm{MRL}=0.01 \mathrm{mg} / \mathrm{L})$, were analyzed using a Dionex Integrion HP ion chromatograph (Thermo Scientific). $\mathrm{pH}$ was measured using an accumet $\mathrm{pH}$ probe (Fisher Scientific), and verified with $\mathrm{pH}$ paper (Hydrion). UV absorbance at $254 \mathrm{~nm}\left(\mathrm{MRL}=0.01 \mathrm{~cm}^{-1}\right.$ ) was measured using an Agilent Cary $60 \mathrm{UV}$-Vis spectrophotometer. The specific UV absorbance (SUVA254) was calculated by dividing the UV absorbance by the DOC.

Text S2. LC-MS/MS methods descriptions. Contaminants of emerging concern were quantified on a LC-MS/MS 6460 triple quadrupole system (Agilent) using a $150 \mathrm{~mm}$ x $3 \mathrm{~mm}$ Synergi $4 \mu \mathrm{m}$ Hydro-RP $80 \AA$ Å column (Phenomenex). Compounds were separated using an 18 minute gradient method that used $0.1 \%$ acetic acid (A) and methanol (B) at a $0.6 \mathrm{~mL} / \mathrm{min}$ flow rate. The gradient used is shown in the Table $\mathrm{S} 2.10 \mu \mathrm{L}$ of sample were injected during each run. Electrospray ionization was used to detect the compounds, with a gas temperature of $300{ }^{\circ} \mathrm{C}$, gas flow rate of $7 \mathrm{~L} / \mathrm{min}$, a nebulizer pressure of $45 \mathrm{psi}$, a $250^{\circ} \mathrm{C}$ sheath gas temperature and $9 \mathrm{~L} / \mathrm{min}$ sheath gas flow rate, a $3500 \mathrm{~V}$ capillary voltage and $500 \mathrm{~V}$ nozzle voltage. 17 compounds were quantified using multiple reaction monitoring (MRM), in a method adapted from McCurry et al. (2014) and Jasper and Sedlak (2013). Compound specific MRM parameters and retention times are listed in Table S3. 
In addition to the 17 compounds quantified in the MRM mode, one compound, sucralose, was quantified using selected ion monitoring (SIM) in the negative mode, in a method adapted from Ferrer et al. (2013). The same method parameters were used as described above, and sucralose, which eluted at a retention time of $7.5 \mathrm{~min}$, was quantified at an $\mathrm{m} / \mathrm{z}$ of 395 . Sucralose-d6 was used as an internal standard and was quantified at an $\mathrm{m} / \mathrm{z}$ of 401 . For both sucralose and the internal standard, the dwell was set to $200 \mathrm{~ms}$, the fragmentor was set to $135 \mathrm{~V}$, and the cell accelerator voltage was set to $7 \mathrm{~V}$.

Text S3. DBP methods descriptions. 25 halogenated DBPs were analyzed using a modified US EPA Method 551.1. $40 \mathrm{~mL}$ samples were extracted into $3 \mathrm{~mL}$ of MtBE (liquid-liquid extraction) spiked with 1,2-dibromopropane as an internal standard. The extract was concentrated to $0.5 \mathrm{~mL}$ by blowing down with nitrogen. Exrtacts were analyzed by gas chromatography and mass spectrometry (GC/MS). The analytes included were:

- Four trihalomethanes (THM4): chloroform (TCM), bromodichloromethane (BDCM), chlorodibromomethane (CDBM), bromoform (TBM)

- Four haloacetonitriles (HANs): dichloroacetonitrile (DCAN), bromochloroacetonitrile (BCAN), dibromoacetonitrile (DBAN), trichloroacetonitrile (TCAN)

- Four haloacetamides (HAMs): dichloroacetamide (DCAM), bromochloroacetamide (BCAM), dibromoacetamied (DBAM), trichloroacetamide (TCAM)

- Four haloacetaldehydes (HALs): trichloroacetaldehyde (TCAL), bromodichloroacetaldehyde (BDCAL), dibromochloroacetaldehyde (DBCAL), tribromoacetaldehyde (TBAL)

- Six iodinated trihalomethanes (I-THMs): dichloroiodomethane (DCIM), bromochloroiodomethane (BCIM), dibromoiodomethane (DBIM), chlorodiiodomethane (CDIM), bromoiiodomethane (BDIM), iodoform (TIM)

- Two haloketones (HKs): 1,1-dichloropropanone (1,1-DCP), 1,1,1-trichloropropanone $(1,1,1-\mathrm{TCP})$

- One halonitromethane (HNM): chloropicrin (TCNM)

Ten haloacetic acids (HAAs) were analyzed using modified US EPA Method 552.3. $40 \mathrm{~mL}$ samples were acidified and extracted into $3 \mathrm{~mL}$ of MtBE (liquid-liquid extraction) spiked with 1,2-dibromopropane as an internal standard. Acidic methanol was used to methylate the samples, the samples were back extracted, concentrated to $0.5 \mathrm{~mL}$ and analyzed by GC/MS. The analytes included were:

- Ten haloacetic acids (HAAs): chloroacetic acid (CAA), bromoacetic acid (BAA), iodoacetic acid (IAA), dichloroacetic acid (DCAA), bromochloroacetic acid (BCAA), dibromoacetic acid (BCAA), dibromoacetic acid (DBAA), trichloroacetic acid (TCAA), bromodichloroacetic acid (BDCAA), dibromochloroacetic acid (DBCAA), tribomoacetic acid (TBAA)

Eight $N$-nitrosamines were analyzed using modified US EPA Method $522.500 \mathrm{~mL}$ samples were spiked with deuterated $N$-nitrosodimethylamine (NDMA) and $N$-nitrosomorpholine (NMOR) as internal standards, and extracted using activated carbon solid phase extraction cartridges. Samples were eluted with methylene chloride, concentrated to $1 \mathrm{~mL}$, and analyzed by GC/MS using methanol chemical ionization. The analytes included were: 
- Eight $N$-nitrosamines (NAs): NDMA, NMOR, $N$-nitrosomethylethylamine (NMEA), $N$-nitrosodiethylamine (NDEA), $N$-nitrosodi-n-propylamine (NDPA), $N$-nitrosodi-nbutylamine (NDBA), $N$-nitrosopyrrolidine (NPYR), $N$-nitrosopiperidine (NPIP).

Text S4. Lab-scale FO membrane experiment descriptions. The rejection of ammonia, chloroform, 1,4-dioxane, and benzotriazole by a Porifera PFO-100 FO membrane coupon was evaluated at lab-scale in a SEPA cell, maintaining a flux between 19.7-22.0 lmh. A $250 \mathrm{~mL}$ solution of $50 \mathrm{mg}-\mathrm{N} / \mathrm{L}$ of ammonia, $200 \mu \mathrm{g} / \mathrm{L}$ chloroform, $1000 \mu \mathrm{g} / \mathrm{L}$ 1,4-dioxane, and $250 \mu \mathrm{g} / \mathrm{L}$ benzotriazole in deionized water was used as the feed stock, a $250 \mathrm{~mL}$ solution of $1 \mathrm{M} \mathrm{NaCl}$ was used to constitute the draw. Duplicate samples were taken for three independent runs after $\sim 10$ $\mathrm{mL}$ of sample passed through the membrane. Ammonia, chloroform, 1,4-dioxane were measured as described in the Materials and Methods section, while benzotriazole was measured using an Agilent 1260 Infinity HPLC with a Poroshell 120 EC-C18 $2.7 \mu \mathrm{m}, 3$ x $50 \mathrm{~mm}$ column. A gradient method was used, with parameters described in Table S2. Benzotriazole was quantified at a $260 \mathrm{~nm} \mathrm{UV}$ absorbance.

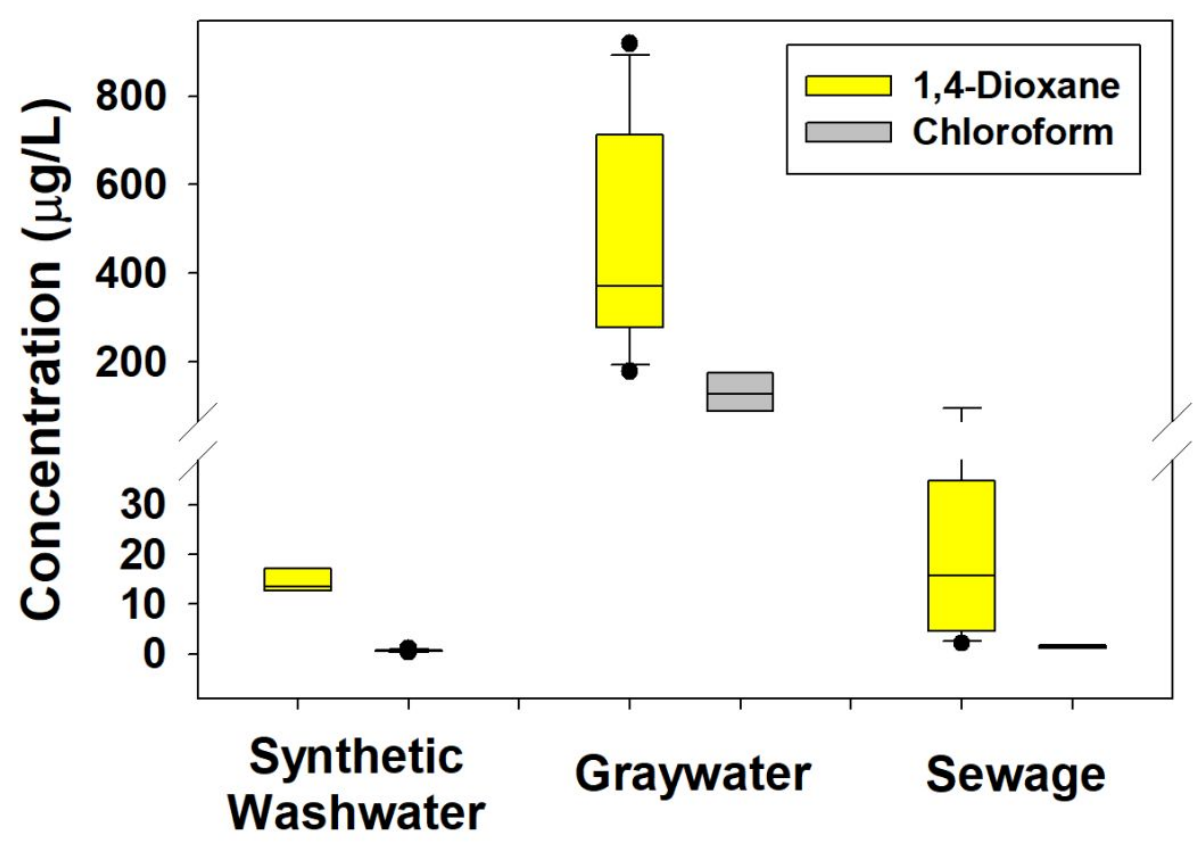

Figure S1. 1,4-Dioxane and chloroform concentrations detected in the synthetic washwater blend, graywater, and sewage. The graywater and sewage samples were sampled four times, each in triplicate. The synthetic washwater was sampled once in triplicate. 



Figure S2. (A) Total coliform and (B) E. coliconcentrations detected in the FO feed and

RO permeate during FO-RO treatment of greywater and sewage. 



Figure S3. Concentrations of (A) total DBPs, (B) chloroform (TCM), and (C) NDMA detected in the FO feed (Feed), FO draw solution (post FO), and RO permeate (post $\mathrm{RO})$ during treatment of graywater and sewage for decentralized reuse applications.

Box plots are based on 4 separate sampling events. 

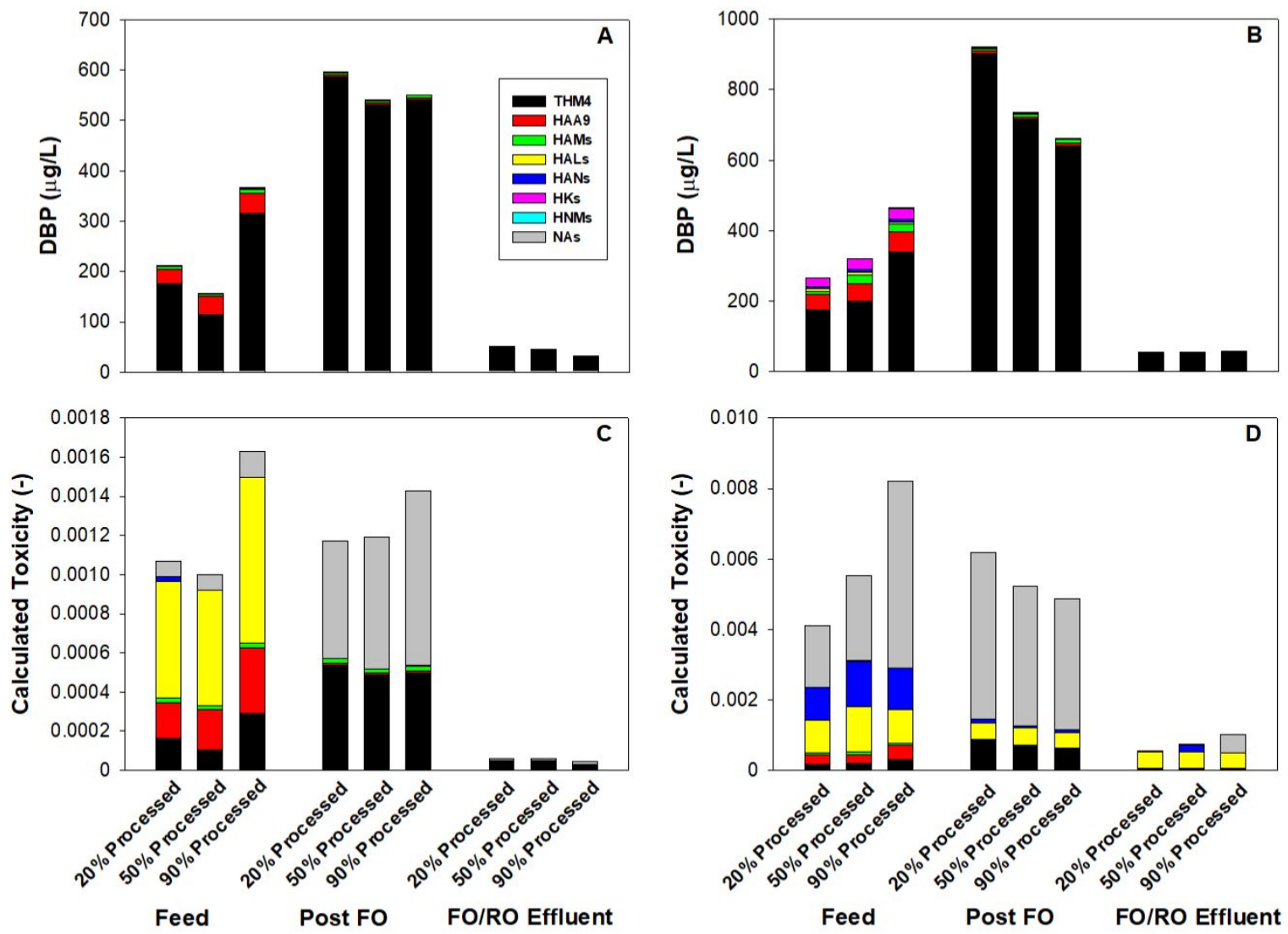

Figure S4. DBP concentrations detected in greywater before (A) and after (B) chloramine treatment alongside corresponding calculated toxicities $(\mathrm{C}+\mathrm{D})$ throughout one sampling event. 

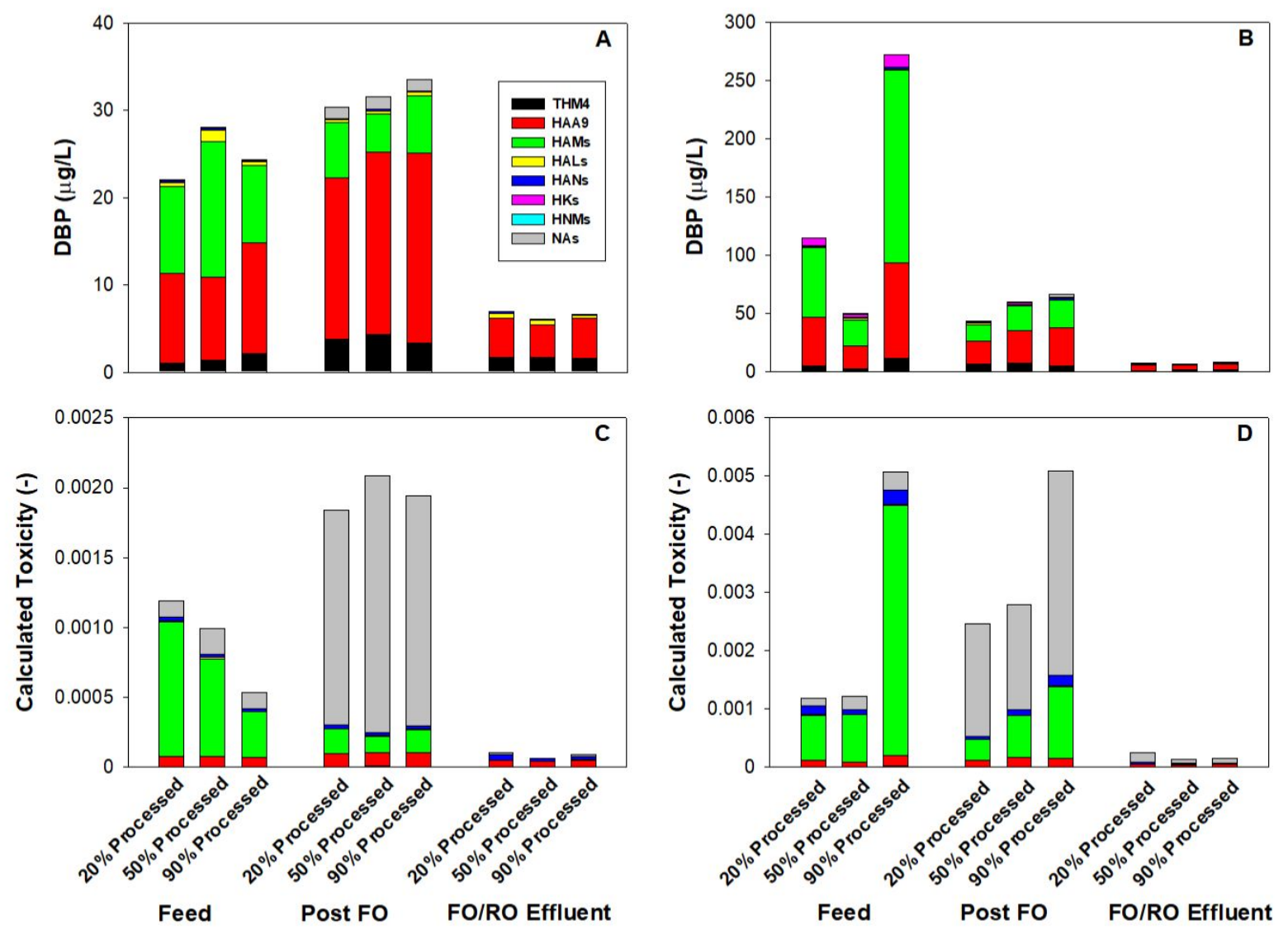

Figure S5. DBP concentrations detected in sewage before (A) and after (B) chloramine treatment alongside corresponding calculated toxicities $(\mathrm{C}+\mathrm{D})$ throughout one sampling event. 


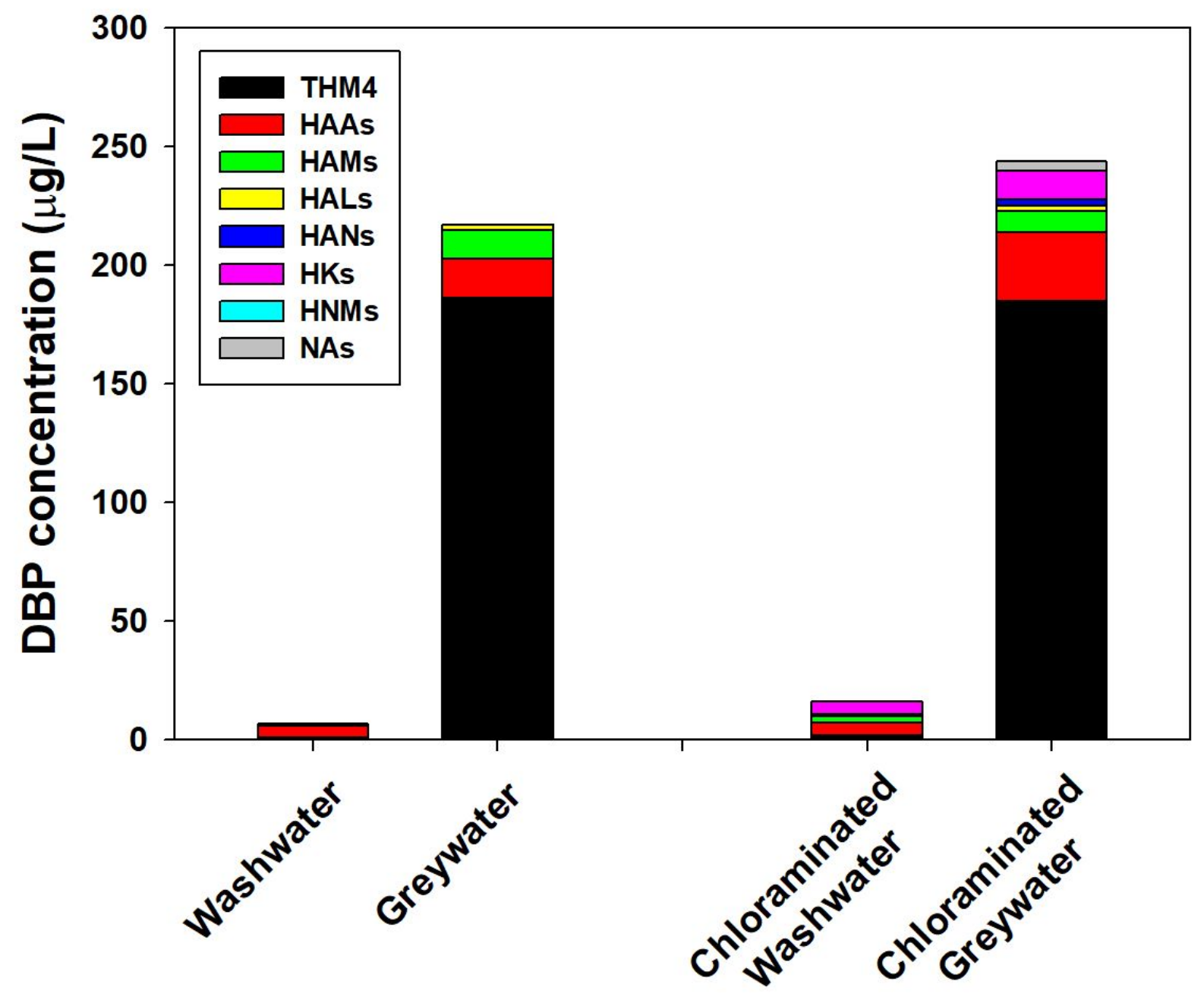

Figure S6. DBP concentrations detected in the synthetic washwater blend and a representative greywater mixture sample (sampling event 3 ) with and without chloramine treatment. 
Table S1. NSF 350-1 Wash Water Formulation.

\begin{tabular}{|c|c|c|}
\hline Wash Water Components & Item used & Amount/100L \\
\hline \hline Body wash with moisturizer & Dial Spring Water Hydrating Body Wash & $30 \mathrm{~g}$ \\
Toothpaste & Colgate Fluoride Toothpaste- regular & $3 \mathrm{~g}$ \\
Deodorant & Secret Powder Fresh antiperspirant & $2 \mathrm{~g}$ \\
Shampoo & Suave Ocean Breeze Shampoo & $19 \mathrm{~g}$ \\
Conditioner & Suave Tropical Coconut Conditioner & $21 \mathrm{~g}$ \\
Lactic acid & $85 \%$ w/w ACS grade (Fisher) & $3 \mathrm{~mL}$ \\
Bath cleaner & Lysol Disinfectant Bathroom Cleaner & $10 \mathrm{~g}$ \\
Liquid hand soap & Dial Gold Antibacterial Hand Soap & $23 \mathrm{~g}$ \\
Test dust & Collected indoor dust & $10 \mathrm{~g}$ \\
Secondary municipal wastewater effluent & Effluent from membrane aerated bioreactor & $2 \mathrm{~L}$ \\
\hline
\end{tabular}

Table S2. Gradient method parameters. A=0.1\% Acetic Acid, B=Methanol.

\begin{tabular}{|c|c|c|}
\hline Time $(\min )$ & $\mathrm{A}(\%)$ & $\mathrm{B}(\%)$ \\
\hline 0 & 95 & 5 \\
2 & 95 & 5 \\
8 & 40 & 60 \\
10 & 40 & 60 \\
12 & 5 & 95 \\
13 & 5 & 95 \\
15 & 95 & 5 \\
18 & 95 & 5 \\
\hline
\end{tabular}

Table S3. LC-MS/MS detection limits.

\begin{tabular}{|c|c|}
\hline Compound & Detection Limit (nM) \\
\hline Naproxen & 0.5 \\
Diuron & 0.5 \\
Carbamazepine & 0.5 \\
Atenolol & 0.5 \\
Hydrochlorothiazide & 0.5 \\
Diclofenac & 0.5 \\
Oryzalin & 0.5 \\
Bezafibrate & 1 \\
Sulfamethoxazole & 1 \\
Sucralose & 1 \\
Benzotriazole & 1 \\
Ibuprofen & 1 \\
Gemfibrozil & 1 \\
Ranitindine & 2 \\
Ciprofloxacin & 2 \\
Fipronil & 2 \\
Acyclovir & 2 \\
\hline
\end{tabular}


Table S4. Mass spectrometry parameters.

\begin{tabular}{|c|c|c|c|c|c|c|c|c|}
\hline Compound & $\begin{array}{l}\text { Retention } \\
\text { Time } \\
(\min )\end{array}$ & $\begin{array}{c}\text { Parent } \\
\text { Ion } \\
(\mathrm{m} / \mathrm{z})\end{array}$ & $\begin{array}{c}\text { Product } \\
\text { Ion }(\mathrm{s}) \\
(\mathrm{m} / \mathrm{z})\end{array}$ & $\begin{array}{l}\text { Dwell } \\
(\mathrm{ms})\end{array}$ & $\begin{array}{l}\text { Fragmentor } \\
\text { (V) }\end{array}$ & $\begin{array}{c}\text { Collision } \\
\text { Energy } \\
\text { (V) }\end{array}$ & $\begin{array}{c}\text { Cell } \\
\text { Accelerator } \\
\text { Voltage (V) }\end{array}$ & Polarity \\
\hline Fipronil & 13.8 & 435 & 330 & 7 & 112 & 8 & 7 & - \\
\hline Fipronil & 13.8 & 435 & 250 & 7 & 112 & 20 & 7 & - \\
\hline Bezafibrate & 11.0 & 362 & 316 & 7 & 140 & 7 & 7 & + \\
\hline Bezafibrate & 11.0 & 362 & 139 & 7 & 140 & 19 & 7 & + \\
\hline Oryzalin & 13.3 & 345 & 281 & 7 & 170 & 10 & 7 & - \\
\hline Oryzalin & 13.3 & 345 & 78 & 7 & 170 & 30 & 7 & - \\
\hline Ciprofloxacin-d4 & 7.1 & 340 & 322 & 7 & 130 & 30 & 7 & + \\
\hline Ciprofloxacin & 7.1 & 332 & 314 & 7 & 130 & 30 & 7 & + \\
\hline Ciprofloxacin & 7.1 & 332 & 231 & 7 & 130 & 34 & 7 & + \\
\hline Ranitidine & 6.0 & 315 & 176 & 7 & 120 & 10 & 7 & + \\
\hline Ranitidine & 6.0 & 315 & 130 & 7 & 120 & 18 & 7 & + \\
\hline Hydrochlorothiazide & 7.5 & 296 & 269 & 7 & 170 & 9 & 7 & - \\
\hline Hydrochlorothiazide & 7.5 & 296 & 205 & 7 & 170 & 13 & 7 & - \\
\hline Diclofenac-d4 & 12.8 & 300 & 218 & 7 & 118 & 29 & 7 & + \\
\hline Diclofenac & 12.8 & 296 & 250 & 7 & 118 & 5 & 7 & + \\
\hline Diclofenac & 12.8 & 296 & 214 & 7 & 118 & 29 & 7 & + \\
\hline Atenolol-d7 & 5.3 & 274 & 145 & 7 & 130 & 24 & 7 & + \\
\hline Atenolol & 5.3 & 267 & 190 & 7 & 130 & 16 & 7 & + \\
\hline Atenolol & 5.3 & 267 & 145 & 7 & 130 & 24 & 7 & + \\
\hline Sulfamethoxazole-d10 & 8.9 & 258 & 96 & 7 & 110 & 25 & 7 & + \\
\hline Sulfamethoxazole & 8.9 & 254 & 156 & 7 & 110 & 10 & 7 & + \\
\hline Sulfamethoxazole & 8.9 & 254 & 92 & 7 & 110 & 25 & 7 & + \\
\hline Gemfibrozil & 13.9 & 249 & 121 & 7 & 120 & 25 & 7 & - \\
\hline Carbamazepine-d10 & 9.8 & 247 & 204 & 7 & 120 & 20 & 7 & + \\
\hline Carbamazepine & 9.8 & 237 & 194 & 7 & 120 & 15 & 7 & + \\
\hline Carbamazepine & 9.8 & 237 & 179 & 7 & 120 & 35 & 7 & + \\
\hline Diuron & 11.0 & 233 & 160 & 7 & 112 & 25 & 7 & + \\
\hline Diuron & 11.0 & 233 & 72 & 7 & 112 & 17 & 7 & + \\
\hline Acyclovir-d4 & 2.5 & 230 & 152 & 7 & 70 & 10 & 2 & + \\
\hline Acyclovir & 2.5 & 226 & 152 & 7 & 70 & 10 & 2 & + \\
\hline Acyclovir & 2.5 & 226 & 152 & 7 & 70 & 45 & 2 & + \\
\hline Naproxen-d3 & 11.1 & 232 & 173 & 7 & 52 & 3 & 7 & - \\
\hline Naproxen & 11.1 & 229 & 170 & 7 & 52 & 3 & 7 & - \\
\hline Naproxen & 11.1 & 229 & 169 & 7 & 52 & 19 & 7 & - \\
\hline Ibuprofen & 6.9 & 207 & 166 & 7 & 61 & 7 & 4 & + \\
\hline Ibuprofen & 6.9 & 207 & 125 & 7 & 61 & 7 & 4 & + \\
\hline Benzotriazole & 7.8 & 120 & 92 & 7 & 74 & 17 & 7 & + \\
\hline Benzotriazole & 7.8 & 120 & 65 & 7 & 74 & 17 & 7 & + \\
\hline
\end{tabular}


Table S5. Structures and molecular volumes of organic contaminants (Royal Society of Chemistry).

\begin{tabular}{|c|c|c|}
\hline Compound & Molecular Volume $\left(\mathrm{cm}^{3}\right)$ & Structure \\
\hline Benzotriazole & 88.3 & \\
\hline 1,4-dioxane & 88.5 & \\
\hline Acyclovir & 127.2 & \\
\hline Sulfamethoxazole & 173.1 & \\
\hline Hydrochlorothiazide & 175.8 & \\
\hline Diuron & 178.6 & \\
\hline Naproxen & 192.3 & \\
\hline Carbamazepine & 193.4 & \\
\hline Ibuprofen & 200.3 & \\
\hline Diclofenac & 206.8 & \\
\hline Ciprofloxacin & 226.8 & \\
\hline
\end{tabular}




Fipronil


Table S6. Chloramine and free chlorine doses for decentralized FO/RO samples.

\begin{tabular}{|c|c|c|}
\hline & Greywater & Sewage \\
\hline \multicolumn{3}{|c|}{ Feed: chloramine doses $\left(\mathrm{mg}-\mathrm{Cl}_{2} / \mathrm{L}\right)$} \\
\hline Event 1 & 20 & 20 \\
\hline Event 2 & 20 & 20 \\
\hline Event 3 & 20 & 20 \\
\hline Event $420 \%$ Processed & 28 & 20 \\
\hline Event $450 \%$ Processed & 30 & 20 \\
\hline Event $490 \%$ Processed & 35 & 20 \\
\hline \multicolumn{3}{|c|}{ Between FO and RO: chloramine doses $\left(\mathrm{mg}-\mathrm{Cl}_{2} / \mathrm{L}\right.$} \\
\hline Event 1 & 15 & 15 \\
\hline Event 2 & 15 & 15 \\
\hline Event 3 & 15 & 15 \\
\hline Event $420 \%$ Processed & 15 & 15 \\
\hline Event $450 \%$ Processed & 15 & 15 \\
\hline Event $490 \%$ Processed & 15 & 15 \\
\hline \multicolumn{3}{|c|}{ RO effluent: chloramine doses $\left(\mathrm{mg}-\mathrm{Cl}_{2} / \mathrm{L}\right)$} \\
\hline Event 1 & 7 & 7 \\
\hline Event 2 & 7 & 7 \\
\hline Event 3 & 7 & 7 \\
\hline Event $420 \%$ Processed & 7 & 7 \\
\hline Event $450 \%$ Processed & 7 & 7 \\
\hline Event $490 \%$ Processed & 7 & 7 \\
\hline \multicolumn{3}{|c|}{$\mathrm{RO}$ effluent: free chlorine doses $\left(\mathrm{mg}-\mathrm{Cl}_{2} / \mathrm{L}\right)$} \\
\hline Event 1 & 12.5 & 37 \\
\hline Event 2 & 28 & 24 \\
\hline Event 3 & 32 & 64 \\
\hline Event $420 \%$ Processed & 15 & 33 \\
\hline Event $450 \%$ Processed & 15 & 25 \\
\hline Event $490 \%$ Processed & 15 & 60 \\
\hline
\end{tabular}


Table S7. $\mathrm{LC}_{50}$ values for the disinfection byproducts detected (Wagner et al., 2017).

\begin{tabular}{|c|c|}
\hline DBP & $\mathrm{LC}_{50}(\mathrm{M})$ \\
\hline \multicolumn{2}{|c|}{ THM4 } \\
\hline TCM & $9.2 \times 10^{-3}$ \\
\hline BDCM & $1.2 \times 10^{-2}$ \\
\hline DBCM & $5.4 \times 10^{-3}$ \\
\hline TBM & $4.0 \times 10^{-3}$ \\
\hline \multicolumn{2}{|c|}{ HAAs } \\
\hline$\overline{\mathrm{CAA}}$ & $8.5 \times 10^{-4}$ \\
\hline BAA & $9.6 \times 10^{-6}$ \\
\hline DCAA & $7.3 \times 10^{-3}$ \\
\hline BCAA & $7.8 \times 10^{-4}$ \\
\hline DBAA & $5.2 \times 10^{-4}$ \\
\hline TCAA & $2.4 \times 10^{-3}$ \\
\hline BDCAA & $6.8 \times 10^{-4}$ \\
\hline CDBAA & $2.0 \times 10^{-4}$ \\
\hline TBAA & $8.5 \times 10^{-5}$ \\
\hline IAA & $3.2 \times 10^{-6}$ \\
\hline \multicolumn{2}{|c|}{ HALs } \\
\hline TCAL & $1.2 \times 10^{-3}$ \\
\hline BDCAL & $2.0 \times 10^{-5}$ \\
\hline DBCAL & $5.1 \times 10^{-6}$ \\
\hline TBAL & $3.6 \times 10^{-6}$ \\
\hline \multicolumn{2}{|c|}{ HANs } \\
\hline TCAN & $21.6 \times 10^{-4}$ \\
\hline DCAN & $5.7 \times 10^{-5}$ \\
\hline BCAN & $8.5 \times 10^{-6}$ \\
\hline DBAN & $2.9 \times 10^{-6}$ \\
\hline \multicolumn{2}{|c|}{ HAMs } \\
\hline TCAM & $2.1 \times 10^{-3}$ \\
\hline DCAM & $1.9 \times 10^{-3}$ \\
\hline BCAM & $1.7 \times 10^{-5}$ \\
\hline DBAM & $1.2 \times 10^{-5}$ \\
\hline \multicolumn{2}{|c|}{ HNMs } \\
\hline TCNM & $5.4 \times 10^{-4}$ \\
\hline \multicolumn{2}{|c|}{ HKs } \\
\hline 1,1-DCP & $(-)$ \\
\hline $1,1,1-\mathrm{TCP}$ & $(-)$ \\
\hline \multicolumn{2}{|c|}{ I-THMs } \\
\hline DCIM & $4.1 \times 10^{-3}$ \\
\hline BCIM & $2.4 \times 10^{-3}$ \\
\hline DBIM & $1.9 \times 10^{-3}$ \\
\hline CDIM & $2.4 \times 10^{-3}$ \\
\hline BDIM & $(-)$ \\
\hline TIM & $6.6 \times 10^{-5}$ \\
\hline
\end{tabular}


Table S8. LECR $_{50}$ values for the disinfection byproducts detected (Chuang et al., 2017).

\begin{tabular}{|c|c|}
\hline DBP & LECR $_{50}(\mathrm{M})$ \\
\hline \hline \multicolumn{2}{|c|}{$N$-Nitrosamines } \\
\hline \hline NDMA & $4.0 \times 10^{-6}$ \\
\hline NMEA & $1.7 \times 10^{-5}$ \\
\hline NDEA & $2.0 \times 10^{-6}$ \\
\hline NDPA & $2.7 \times 10^{-5}$ \\
\hline NPYR & $1.0 \times 10^{-5}$ \\
\hline NPIP & $1.5 \times 10^{-5}$ \\
\hline NDBA & $9.5 \times 10^{-5}$ \\
\hline NMOR & $2.2 \times 10^{-5}$ \\
\hline
\end{tabular}


Table S9. Mean (minimum - maximum) of concentrations of general water quality parameters measured during four

sample events for centralized RO treatment of microfiltered secondary effluent and FO-RO treatment of RO concentrate.

\begin{tabular}{|c|c|c|c|c|}
\hline & \multicolumn{2}{|c|}{ FO-RO treating RO concentrate } & \multicolumn{2}{c|}{ RO treating MF effluent } \\
\hline \hline & Feed & Permeate & Feed & Permeate \\
\hline \hline TOC (mg-C/L) & $40.8(35.6-45.9)$ & $0.4(0.2-0.8)$ & $7.8^{*}$ & $0.1^{*}$ \\
TDS (mg/L) & $6180(6170-6190)$ & $200(92-310)$ & $1020^{*}$ & $1^{*}$ \\
Conductivity $(\mu \mathrm{S} / \mathrm{cm})$ & $\mathbf{8 8 4 0 ( 8 8 3 0 - 8 8 5 0 )}$ & $430(230-600)$ & $1730^{*}$ & $100^{*}$ \\
$\mathrm{NO}_{3}^{-}(\mathrm{mg}-\mathrm{N} / \mathrm{L})$ & $58(50-63)$ & $1.4(1.0-3.0)$ & $10(10-10)$ & $0.6(0.6-0.7)$ \\
$\mathrm{NO}_{2}^{-}(\mathrm{mg}-\mathrm{N} / \mathrm{L})$ & $3.2(0.8-7.6)$ & $<0.02$ & $0.1(<0.02-0.1)$ & $<0.02$ \\
$\mathrm{Br}^{-}(\mathrm{mg} / \mathrm{L})$ & $2.3(1.9-2.6)$ & $<0.1$ & $0.3(0.3-0.3)$ & $<0.1$ \\
$\mathrm{Cl}^{-}(\mathrm{mg} / \mathrm{L})$ & $1830(1630-1970)$ & $110(30-240)$ & $\mathbf{2 6 0 ( 2 4 0 - 2 8 0 )}$ & $4.1(3.5-4.7)$ \\
\hline
\end{tabular}

* = Historical values measured by the utility. 
Table S10. Mean (minimum - maximum) concentrations of general water quality parameters measured during six sample

events for decentralized FO-RO treatment of graywater and sewage.

\begin{tabular}{|c|c|c|c|c|c|c|}
\hline & \multicolumn{3}{|c|}{ Graywater } & \multicolumn{3}{|c|}{ Sewage } \\
\hline & Feed & Between FO and RO & Permeate & Feed & Between FO and RO & Permeate \\
\hline COD (mg/L) & $1290(915-1660)$ & - & $5.8(2.7-9.3)$ & $1040(610-2100)$ & - & $6.5(4.3-9.8)$ \\
\hline DOC (mg-C/L) & $160(100-310)$ & $17(9.8-25)$ & $0.3(<0.1-0.7)$ & $84(44-120)$ & $20(14-27)$ & $0.2(<0.1-0.5)$ \\
\hline BOD5 (mg/L) & $1210(760-1200)$ & - & $4.5(0.4-7.2)$ & $1030(350-1840)$ & - & $0.1(<0.1-0.3)$ \\
\hline TSS (mg/L) & $48(38-70)$ & - & $<0.1$ & $67(43-93)$ & - & $0.1(<0.1-0.3)$ \\
\hline TP (mg-P/L) & $3.0(1.1-6.7)$ & - & $<0.1$ & $16(10-25)$ & - & $<0.1$ \\
\hline $\mathrm{NH}_{3}(\mathrm{mg}-\mathrm{N} / \mathrm{L})$ & $14(9-24)$ & $140(97-240)$ & $1.1(0.03-3.4)$ & $66(49-77)$ & $390(280-520)$ & $4.3(2.7-7.6)$ \\
\hline Conductivity $(\mu \mathrm{S} / \mathbf{c m})$ & $1490(860-2150)$ & $50000(49000-51000)$ & $340(77-640)$ & $1960(920-3500)$ & $50000(49000-51000)$ & $440(120-750)$ \\
\hline Turbidity (NTU) & $140(69-230)$ & - & $<0.2$ & $180(83-240)$ & - & $<0.2$ \\
\hline $\mathrm{NO}_{3}{ }^{-}(\mathrm{mg}-\mathrm{N} / \mathrm{L})$ & $2.8(0.8-4.3)$ & - & $<0.2$ & $1.3(0.8-2.3)$ & - & $0.3(<0.2-0.5)$ \\
\hline $\mathrm{NO}_{2}^{-}(\mathrm{mg}-\mathrm{N} / \mathrm{L})$ & $0.85(0.32-1.9)$ & - & $0.02(<0.02-0.03)$ & $0.24(0.14-0.43)$ & - & $0.02(<0.02-0.03)$ \\
\hline $\mathrm{Br}^{-}(\mathrm{mg} / \mathrm{L})$ & $0.4(0.3-0.7)$ & $1.0(0.8-1.3)$ & $<0.1$ & $0.1(<0.1-0.2)$ & $0.4(0.3-0.5)$ & $<0.1$ \\
\hline $\mathrm{Cl}^{-}(\mathrm{mg} / \mathrm{L})$ & $160(87-330)$ & $14300(11100-19500)$ & $25(16-38)$ & $110(82-190)$ & $23900(17800-34100)$ & $49(37-67)$ \\
\hline pH & $6.7(5.8-7.2)$ & $5.6(3.3-6.5)$ & $4.4(2.9-5.3)$ & $7.2(6.9-7.6)$ & $5.0(2.7-7.2)$ & $5.8(4.9-6.5)$ \\
\hline UV254 $\left(\mathrm{cm}^{-1}\right)$ & $0.53(0.23-0.88)$ & $0.15(0.09-0.26)$ & $<0.01$ & $0.54(0.36-0.76)$ & $0.19(0.10-0.29)$ & $<0.01$ \\
\hline SUVA $\left(\mathbf{L} \mathbf{m g}^{-1} \mathbf{m}^{-1}\right)$ & $3.2(2.3-4.1)$ & $8.4(5.9-11)$ & $\mathbf{N} / \mathbf{A}$ & $6.7(4.6-9.0)$ & $11(4.4-17)$ & $\mathbf{N} / \mathbf{A}$ \\
\hline
\end{tabular}


Tables S11-S38. DBPs detected during decentralized treatment of greywater and sewage. ITHMs were not detected. Error bars represent standard deviations for triplicate measurements for halogenated DBPs, and ranges for duplicate measurements for $N$-nitrosamines. 
Table S11. HAAs detected in samples collected during decentralized treatment of greywater.

\begin{tabular}{|c|c|c|c|c|c|c|c|c|c|c|}
\hline Sample & $\mathrm{CAA}$ & $\mathrm{BAA}$ & DCAA & BCAA & DBAA & TCAA & BDCAA & CDBAA & TBAA & IAA \\
\hline \multicolumn{11}{|c|}{ Feed } \\
\hline Event 1 & $2.7 \pm 0.4$ & $<0.20$ & $13.9 \pm 2.2$ & $<0.16$ & $0.25 \pm 0.10$ & $10.9 \pm 0.9$ & $<0.22$ & $3.4 \pm 0.3$ & $4.1 \pm 0.7$ & $<0.20$ \\
\hline Event 2 & $2.5 \pm 0.2$ & $<0.20$ & $8.3 \pm 1.4$ & $<0.16$ & $0.46 \pm 0.04$ & $10.0 \pm 1.9$ & $<0.22$ & $2.8 \pm 0.5$ & $1.0 \pm 0.05$ & $<0.20$ \\
\hline Event 3 & $1.6 \pm 0.3$ & $<0.20$ & $7.1 \pm 0.4$ & $<0.16$ & $0.18 \pm 0.01$ & $3.1 \pm 0.6$ & $0.7 \pm 0.1$ & $3.3 \pm 0.2$ & $0.63 \pm 0.07$ & $<0.20$ \\
\hline Event $420 \%$ Processed & $2.0 \pm 0.2$ & $<0.20$ & $13.8 \pm 0.1$ & $<0.16$ & $0.39 \pm 0.02$ & $7.3 \pm 0.1$ & $0.86 \pm 0.16$ & $3.5 \pm 0.1$ & $1.2 \pm 0.1$ & $<0.20$ \\
\hline Event $450 \%$ Processed & $2.6 \pm 0.4$ & $<0.20$ & $16.9 \pm 2.1$ & $<0.16$ & $0.41 \pm 0.12$ & $10.0 \pm 2.0$ & $0.61 \pm 0.12$ & $3.8 \pm 0.7$ & $1.2 \pm 0.3$ & $<0.20$ \\
\hline Event 4 90\% Processed & $4.1 \pm 0.4$ & $<0.20$ & $21.1 \pm 1.0$ & $<0.16$ & $0.18 \pm 0.01$ & $6.3 \pm 0.4$ & $<0.22$ & $6.5 \pm 0.5$ & $3.0 \pm 0.7$ & $<0.20$ \\
\hline \multicolumn{11}{|c|}{ Between FO and RO } \\
\hline Event 1 & $0.35 \pm 0.10$ & $<0.20$ & $1.9 \pm 0.2$ & $<0.16$ & $<0.18$ & $0.47 \pm 0.09$ & $<0.22$ & $<0.22$ & $<0.24$ & $<0.20$ \\
\hline Event 2 & $0.74 \pm 0.24$ & $<0.20$ & $4.0 \pm 0.2$ & $<0.16$ & $<0.18$ & $0.65 \pm 0.02$ & $<0.22$ & $<0.22$ & $<0.24$ & $<0.20$ \\
\hline Event 3 & $0.59 \pm 0.10$ & $<0.20$ & $4.7 \pm 0.3$ & $<0.16$ & $<0.18$ & $0.75 \pm 0.08$ & $<0.22$ & $<0.22$ & $<0.24$ & $<0.20$ \\
\hline Event $420 \%$ Processed & $0.42 \pm 0.07$ & $<0.20$ & $2.7 \pm 0.2$ & $<0.16$ & $<0.18$ & $0.56 \pm 0.03$ & $<0.22$ & $<0.22$ & $<0.24$ & $<0.20$ \\
\hline Event $450 \%$ Processed & $0.58 \pm 0.04$ & $<0.20$ & $2.6 \pm 0.1$ & $<0.16$ & $<0.18$ & $0.49 \pm 0.12$ & $<0.22$ & $<0.22$ & $<0.24$ & $<0.20$ \\
\hline Event 4 90\% Processed & $0.58 \pm 0.08$ & $<0.20$ & $3.1 \pm 0.3$ & $<0.16$ & $<0.18$ & $0.57 \pm 0.04$ & $<0.22$ & $<0.22$ & $<0.24$ & $<0.20$ \\
\hline \multicolumn{11}{|c|}{ RO effluent } \\
\hline Event 1 & $<0.22$ & $<0.20$ & $<0.17$ & $<0.16$ & $<0.18$ & $<0.18$ & $<0.22$ & $<0.22$ & $<0.24$ & $<0.20$ \\
\hline Event 2 & $<0.22$ & $<0.20$ & $<0.17$ & $<0.16$ & $<0.18$ & $<0.18$ & $<0.22$ & $<0.22$ & $<0.24$ & $<0.20$ \\
\hline Event 3 & $<0.22$ & $<0.20$ & $0.36 \pm 0.02$ & $<0.16$ & $<0.18$ & $0.34 \pm 0.03$ & $<0.22$ & $<0.22$ & $<0.24$ & $<0.20$ \\
\hline Event $420 \%$ Processed & $<0.22$ & $<0.20$ & $<0.17$ & $<0.16$ & $<0.18$ & $<0.18$ & $<0.22$ & $<0.22$ & $<0.24$ & $<0.20$ \\
\hline Event $450 \%$ Processed & $0.22 \pm 0.02$ & $<0.20$ & $0.49 \pm 0.01$ & $<0.16$ & $<0.18$ & $0.76 \pm 0.08$ & $<0.22$ & $<0.22$ & $<0.24$ & $<0.20$ \\
\hline Event $490 \%$ Processed & $<0.22$ & $<0.20$ & $0.60 \pm 0.12$ & $<0.16$ & $<0.18$ & $<0.18$ & $<0.22$ & $<0.22$ & $<0.24$ & $<0.20$ \\
\hline MRL* & 0.22 & 0.20 & 0.17 & 0.16 & 0.18 & 0.18 & 0.22 & 0.22 & 0.24 & 0.20 \\
\hline
\end{tabular}

Table S12. HAAs detected after chloramine or chlorine treatment in samples collected during decentralized treatment of greywater.

\begin{tabular}{|c|c|c|c|c|c|c|c|c|c|c|}
\hline Sample & CAA & BAA & DCAA & BCAA & DBAA & TCAA & BDCAA & CDBAA & TBAA & IAA \\
\hline \multicolumn{11}{|c|}{ Feed: Chloramines } \\
\hline Event 1 & $3.6 \pm 0.4$ & $<0.20$ & $29.2 \pm 0.9$ & $<0.16$ & $0.41 \pm 0.03$ & $12.4 \pm 1.2$ & $<0.22$ & $1.5 \pm 0.3$ & $1.4 \pm 0.2$ & $<0.20$ \\
\hline Event 2 & $4.7 \pm 0.8$ & $<0.20$ & $25.9 \pm 3.2$ & $<0.16$ & $0.38 \pm 0.08$ & $4.8 \pm 0.8$ & $<0.22$ & $1.6 \pm 0.1$ & $15.5 \pm 0.5$ & $<0.20$ \\
\hline Event 3 & $2.8 \pm 0.2$ & $<0.20$ & $19.4 \pm 3.3$ & $<0.16$ & $0.26 \pm 0.02$ & $3.6 \pm 0.8$ & $<0.22$ & $2.0 \pm 0.1$ & $1.3 \pm 0.1$ & $<0.20$ \\
\hline Event $420 \%$ Processed & $4.0 \pm 0.5$ & $<0.20$ & $25.9 \pm 1.2$ & $<0.16$ & $0.36 \pm 0.02$ & $3.9 \pm 0.3$ & $<0.22$ & $6.3 \pm 0.3$ & $1.8 \pm 0.2$ & $<0.20$ \\
\hline Event $450 \%$ Processed & $3.9 \pm 0.9$ & $<0.20$ & $33.2 \pm 3.2$ & $<0.16$ & $0.39 \pm 0.02$ & $5.3 \pm 1.2$ & $<0.22$ & $3.1 \pm 0.5$ & $2.1 \pm 0.1$ & $<0.20$ \\
\hline Event $490 \%$ Processed & $7.8 \pm 1.1$ & $<0.20$ & $36.9 \pm 5.1$ & $<0.16$ & $0.81 \pm 0.11$ & $5.5 \pm 0.4$ & $<0.22$ & $4.2 \pm 0.2$ & $3.7 \pm 0.3$ & $<0.20$ \\
\hline \multicolumn{11}{|c|}{ Between FO and RO: Chloramines } \\
\hline Event 1 & $0.77 \pm 0.21$ & $<0.20$ & $4.2 \pm 0.3$ & $<0.16$ & $<0.18$ & $0.56 \pm 0.07$ & $<0.22$ & $<0.22$ & $<0.24$ & $<0.20$ \\
\hline Event 2 & $1.4 \pm 0.2$ & $<0.20$ & $9.3 \pm 0.4$ & $<0.16$ & $<0.18$ & $1.0 \pm 0.1$ & $<0.22$ & $<0.22$ & $<0.24$ & $<0.20$ \\
\hline Event 3 & $1.6 \pm 0.2$ & $<0.20$ & $9.2 \pm 0.5$ & $<0.16$ & $<0.18$ & $1.0 \pm 0.1$ & $<0.22$ & $<0.22$ & $<0.24$ & $<0.20$ \\
\hline Event $420 \%$ Processed & $1.1 \pm 0.3$ & $<0.20$ & $5.2 \pm 0.5$ & $<0.16$ & $<0.18$ & $0.78 \pm 0.11$ & $<0.22$ & $<0.22$ & $<0.24$ & $<0.20$ \\
\hline Event $450 \%$ Processed & $0.95 \pm 0.18$ & $<0.20$ & $4.8 \pm 0.5$ & $<0.16$ & $<0.18$ & $0.68 \pm 0.22$ & $<0.22$ & $<0.22$ & $<0.24$ & $<0.20$ \\
\hline Event $490 \%$ Processed & $1.1 \pm 0.1$ & $<0.20$ & $6.6 \pm 0.5$ & $<0.16$ & $<0.18$ & $0.79 \pm 0.15$ & $<0.22$ & $<0.22$ & $<0.24$ & $<0.20$ \\
\hline \multicolumn{11}{|c|}{ RO effluent: Chloramines } \\
\hline Event 1 & $<0.22$ & $<0.20$ & $0.20 \pm 0.03$ & $<0.16$ & $<0.18$ & $<0.18$ & $<0.22$ & $<0.22$ & $<0.24$ & $<0.20$ \\
\hline Event 2 & $<0.22$ & $<0.20$ & $1.3 \pm 0.1$ & $<0.16$ & $<0.18$ & $1.5 \pm 0.2$ & $<0.22$ & $<0.22$ & $<0.24$ & $<0.20$ \\
\hline Event 3 & $<0.22$ & $<0.20$ & $3.6 \pm 0.2$ & $<0.16$ & $<0.18$ & $2.2 \pm 0.1$ & $<0.22$ & $<0.22$ & $<0.24$ & $<0.20$ \\
\hline Event $420 \%$ Processed & $<0.22$ & $<0.20$ & $<0.17$ & $<0.16$ & $<0.18$ & $<0.18$ & $<0.22$ & $<0.22$ & $<0.24$ & $<0.20$ \\
\hline Event $450 \%$ Processed & $<0.22$ & $<0.20$ & $1.6 \pm 0.1$ & $<0.16$ & $<0.18$ & $0.83 \pm 0.14$ & $<0.22$ & $<0.22$ & $<0.24$ & $<0.20$ \\
\hline Event $490 \%$ Processed & $1.0 \pm 0.1$ & $<0.20$ & $1.0 \pm 0.2$ & $<0.16$ & $<0.18$ & $0.93 \pm 0.07$ & $<0.22$ & $<0.22$ & $<0.24$ & $<0.20$ \\
\hline \multicolumn{11}{|c|}{ RO effluent: Free Chlorine } \\
\hline Event 1 & $0.49 \pm 0.12$ & $<0.20$ & $3.4 \pm 0.2$ & $<0.16$ & $<0.18$ & $2.1 \pm 0.1$ & $<0.22$ & $<0.22$ & $<0.24$ & $<0.20$ \\
\hline Event 2 & $0.31 \pm 0.11$ & $<0.20$ & $1.9 \pm 0.1$ & $<0.16$ & $<0.18$ & $2.5 \pm 0.1$ & $<0.22$ & $1.0 \pm 0.2$ & $<0.24$ & $<0.20$ \\
\hline Event 3 & $2.5 \pm 0.4$ & $<0.20$ & $9.6 \pm 0.5$ & $<0.16$ & $<0.18$ & $4.0 \pm 0.3$ & $<0.22$ & $<0.22$ & $<0.24$ & $<0.20$ \\
\hline Event $420 \%$ Processed & $0.76 \pm 0.04$ & $<0.20$ & $3.3 \pm 0.1$ & $<0.16$ & $<0.18$ & $1.2 \pm 0.1$ & $<0.22$ & $<0.22$ & $<0.24$ & $<0.20$ \\
\hline Event $450 \%$ Processed & $0.51 \pm 0.12$ & $<0.20$ & $2.3 \pm 0.1$ & $<0.16$ & $<0.18$ & $1.3 \pm 0.3$ & $<0.22$ & $0.53 \pm 0.07$ & $<0.24$ & $<0.20$ \\
\hline Event $490 \%$ Processed & $1.5 \pm 0.2$ & $<0.20$ & $15.8 \pm 0.8$ & $<0.16$ & $<0.18$ & $14.7 \pm 1.0$ & $<0.22$ & $<0.22$ & $<0.24$ & $<0.20$ \\
\hline MRL* & 0.22 & 0.20 & 0.17 & 0.16 & 0.18 & 0.18 & 0.22 & 0.22 & 0.24 & 0.20 \\
\hline
\end{tabular}


Table S13. HAAs detected in samples collected during decentralized treatment of sewage.

\begin{tabular}{|c|c|c|c|c|c|c|c|c|c|c|}
\hline Sample & CAA & BAA & DCAA & BCAA & DBAA & TCAA & BDCAA & CDBAA & TBAA & IAA \\
\hline \hline \multicolumn{10}{|c|}{ Feed } \\
\hline Event 1 & $3.4 \pm 0.4$ & $<0.20$ & $2.3 \pm 0.3$ & $1.6 \pm 0.1$ & $<0.18$ & $6.7 \pm 0.5$ & $<0.22$ & $<0.22$ & $1.7 \pm 0.1$ & $<0.20$ \\
Event 2 & $2.7 \pm 0.5$ & $<0.20$ & $2.5 \pm 0.2$ & $1.1 \pm 0.1$ & $<0.18$ & $5.6 \pm 0.3$ & $<0.22$ & $<0.22$ & $1.5 \pm 0.3$ & $<0.20$ \\
Event 3 & $3.1 \pm 0.5$ & $<0.20$ & $2.5 \pm 0.2$ & $1.5 \pm 0.1$ & $<0.18$ & $4.7 \pm 0.9$ & $<0.22$ & $<0.22$ & $0.52 \pm 0.12$ & $<0.20$ \\
Event 4 20\% Processed & $2.6 \pm 0.3$ & $<0.20$ & $1.3 \pm 0.1$ & $1.8 \pm 0.2$ & $<0.18$ & $4.3 \pm 0.4$ & $<0.22$ & $<0.22$ & $0.35 \pm 0.06$ & $<0.20$ \\
Event 4 50\% Processed & $3.2 \pm 0.7$ & $<0.20$ & $1.5 \pm 0.4$ & $1.1 \pm 0.2$ & $<0.18$ & $3.2 \pm 0.7$ & $<0.22$ & $<0.22$ & $0.40 \pm 0.11$ & $<0.20$ \\
Event 4 90\% Processed & $3.0 \pm 0.4$ & $<0.20$ & $1.7 \pm 0.2$ & $1.0 \pm 0.1$ & $<0.18$ & $6.9 \pm 1.1$ & $<0.22$ & $<0.22$ & $<0.24$ & $<0.20$ \\
\hline \multicolumn{8}{|c|}{ Between FO and RO } \\
\hline Event 1 & $1.5 \pm 0.1$ & $<0.20$ & $8.1 \pm 0.2$ & $4.3 \pm 1.0$ & $0.48 \pm 0.05$ & $3.8 \pm 0.6$ & $<0.22$ & $<0.22$ & $<0.24$ & $<0.20$ \\
Event 2 & $2.5 \pm 0.4$ & $<0.20$ & $7.9 \pm 0.3$ & $2.9 \pm 0.1$ & $0.38 \pm 0.03$ & $4.1 \pm 0.7$ & $<0.22$ & $<0.22$ & $<0.24$ & $<0.20$ \\
Event 3 & $4.4 \pm 0.4$ & $<0.20$ & $8.0 \pm 0.3$ & $3.5 \pm 1.6$ & $0.48 \pm 0.05$ & $4.9 \pm 1.0$ & $<0.22$ & $<0.22$ & $<0.24$ & $<0.20$ \\
Event 4 20\% Processed & $3.7 \pm 0.4$ & $<0.20$ & $6.4 \pm 0.5$ & $2.9 \pm 0.3$ & $0.39 \pm 0.07$ & $5.0 \pm 0.8$ & $<0.22$ & $<0.22$ & $<0.24$ & $<0.20$ \\
Event 4 50\% Processed & $4.0 \pm 0.8$ & $<0.20$ & $8.7 \pm 1.7$ & $2.8 \pm 0.4$ & $0.30 \pm 0.10$ & $5.0 \pm 1.7$ & $<0.22$ & $<0.22$ & $<0.24$ & $<0.20$ \\
Event 4 90\% Processed & $3.8 \pm 0.6$ & $<0.20$ & $9.4 \pm 0.6$ & $3.2 \pm 0.6$ & $0.38 \pm 0.10$ & $5.0 \pm 0.4$ & $<0.22$ & $<0.22$ & $<0.24$ & $<0.20$ \\
\hline \multicolumn{70}{|c|}{ RO effluent } \\
\hline Event 1 & $3.4 \pm 0.4$ & $<0.20$ & $<0.17$ & $0.58 \pm 0.19$ & $<0.18$ & $0.44 \pm 0.02$ & $<0.22$ & $<0.22$ & $<0.24$ & $<0.20$ \\
Event 2 & $3.9 \pm 0.6$ & $<0.20$ & $<0.17$ & $0.90 \pm 0.09$ & $<0.18$ & $0.69 \pm 0.10$ & $<0.22$ & $<0.22$ & $<0.24$ & $<0.20$ \\
Event 3 & $2.3 \pm 0.3$ & $<0.20$ & $<0.17$ & $0.64 \pm 0.25$ & $<0.18$ & $0.41 \pm 0.04$ & $<0.22$ & $<0.22$ & $<0.24$ & $<0.20$ \\
Event 4 20\% Processed & $3.4 \pm 0.4$ & $<0.20$ & $0.27 \pm 0.02$ & $0.45 \pm 0.02$ & $<0.18$ & $0.39 \pm 0.03$ & $<0.22$ & $<0.22$ & $<0.24$ & $<0.20$ \\
Event 4 50\% Processed & $2.4 \pm 0.5$ & $<0.20$ & $<0.17$ & $0.80 \pm 0.13$ & $<0.18$ & $0.59 \pm 0.07$ & $<0.22$ & $<0.22$ & $<0.24$ & $<0.20$ \\
Event 4 90\% Processed & $3.4 \pm 1.4$ & $<0.20$ & $<0.17$ & $0.87 \pm 0.05$ & $<0.18$ & $0.39 \pm 0.08$ & $<0.22$ & $<0.22$ & $<0.24$ & $<0.20$ \\
\hline \hline
\end{tabular}

Table S14. HAAs detected after chloramine or chlorine treatment in samples collected during decentralized treatment of sewage.

\begin{tabular}{|c|c|c|c|c|c|c|c|c|c|c|}
\hline Sample & CAA & BAA & DCAA & BCAA & DBAA & TCAA & BDCAA & CDBAA & TBAA & IAA \\
\hline \multicolumn{11}{|c|}{ Feed: Chloramines } \\
\hline Event 1 & $5.5 \pm 0.9$ & $<0.20$ & $48.1 \pm 5.0$ & $1.5 \pm 0.3$ & $<0.18$ & $5.8 \pm 0.5$ & $<0.22$ & $<0.22$ & $1.8 \pm 0.2$ & $<0.20$ \\
\hline Event 2 & $5.2 \pm 0.7$ & $<0.20$ & $42.9 \pm 2.5$ & $1.4 \pm 0.2$ & $<0.18$ & $5.7 \pm 1.0$ & $<0.22$ & $<0.22$ & $1.3 \pm 0.1$ & $<0.20$ \\
\hline Event 3 & $5.9 \pm 1.4$ & $<0.20$ & $36.5 \pm 1.7$ & $0.84 \pm 0.26$ & $<0.18$ & $4.5 \pm 0.4$ & $<0.22$ & $<0.22$ & $0.52 \pm 0.04$ & $<0.20$ \\
\hline Event $420 \%$ Processed & $3.1 \pm 0.8$ & $<0.20$ & $33.8 \pm 3.2$ & $1.16 \pm 0.27$ & $<0.18$ & $3.8 \pm 0.5$ & $<0.22$ & $<0.22$ & $0.41 \pm 0.05$ & $<0.20$ \\
\hline Event $450 \%$ Processed & $2.7 \pm 0.5$ & $<0.20$ & $12.1 \pm 1.9$ & $0.58 \pm 0.21$ & $<0.18$ & $3.7 \pm 0.4$ & $<0.22$ & $<0.22$ & $0.50 \pm 0.04$ & $<0.20$ \\
\hline Event $490 \%$ Processed & $6.4 \pm 1.1$ & $<0.20$ & $67.6 \pm 8.7$ & $1.3 \pm 0.2$ & $<0.18$ & $5.9 \pm 1.2$ & $<0.22$ & $<0.22$ & $0.27 \pm 0.05$ & $<0.20$ \\
\hline \multicolumn{11}{|c|}{ Between FO and RO: Chloramines } \\
\hline Event 1 & $1.8 \pm 0.6$ & $<0.20$ & $7.8 \pm 0.6$ & $4.4 \pm 0.8$ & $0.43 \pm 0.06$ & $3.4 \pm 0.4$ & $<0.22$ & $<0.22$ & $<0.24$ & $<0.20$ \\
\hline Event 2 & $5.3 \pm 0.2$ & $<0.20$ & $8.6 \pm 0.3$ & $3.3 \pm 0.6$ & $0.42 \pm 0.01$ & $4.5 \pm 0.7$ & $<0.22$ & $<0.22$ & $<0.24$ & $<0.20$ \\
\hline Event 3 & $4.4 \pm 1.0$ & $<0.20$ & $8.3 \pm 0.1$ & $3.5 \pm 0.6$ & $0.48 \pm 0.04$ & $4.3 \pm 1.0$ & $<0.22$ & $<0.22$ & $<0.24$ & $<0.20$ \\
\hline Event $420 \%$ Processed & $5.3 \pm 0.8$ & $<0.20$ & $7.8 \pm 1.0$ & $2.6 \pm 0.6$ & $0.26 \pm 0.03$ & $3.5 \pm 0.4$ & $<0.22$ & $<0.22$ & $<0.24$ & $<0.20$ \\
\hline Event $450 \%$ Processed & $8.4 \pm 2.0$ & $<0.20$ & $11.5 \pm 1.1$ & $2.9 \pm 0.6$ & $0.35 \pm 0.01$ & $4.7 \pm 0.6$ & $<0.22$ & $<0.22$ & $<0.24$ & $<0.20$ \\
\hline Event $490 \%$ Processed & $6.1 \pm 1.2$ & $<0.20$ & $15.3 \pm 0.3$ & $5.2 \pm 1.4$ & $0.54 \pm 0.02$ & $5.6 \pm 0.4$ & $<0.22$ & $<0.22$ & $<0.24$ & $<0.20$ \\
\hline \multicolumn{11}{|c|}{ RO effluent: Chloramines } \\
\hline Event 1 & $4.8 \pm 0.6$ & $<0.20$ & $0.20 \pm 0.02$ & $0.75 \pm 0.22$ & $<0.18$ & $0.33 \pm 0.07$ & $<0.22$ & $<0.22$ & $<0.24$ & $<0.20$ \\
\hline Event 2 & $3.8 \pm 0.8$ & $<0.20$ & $0.41 \pm 0.10$ & $1.2 \pm 0.4$ & $<0.18$ & $0.48 \pm 0.09$ & $<0.22$ & $<0.22$ & $<0.24$ & $<0.20$ \\
\hline Event 3 & $2.6 \pm 0.4$ & $<0.20$ & $0.21 \pm 0.02$ & $0.74 \pm 0.09$ & $<0.18$ & $0.66 \pm 0.25$ & $<0.22$ & $<0.22$ & $<0.24$ & $<0.20$ \\
\hline Event $420 \%$ Processed & $3.3 \pm 0.2$ & $<0.20$ & $0.30 \pm 0.03$ & $0.73 \pm 0.22$ & $<0.18$ & $0.47 \pm 0.11$ & $<0.22$ & $<0.22$ & $<0.24$ & $<0.20$ \\
\hline Event $450 \%$ Processed & $2.7 \pm 0.4$ & $<0.20$ & $0.28 \pm 0.04$ & $0.79 \pm 0.08$ & $<0.18$ & $0.36 \pm 0.01$ & $<0.22$ & $<0.22$ & $<0.24$ & $<0.20$ \\
\hline Event $490 \%$ Processed & $3.1 \pm 0.6$ & $<0.20$ & $0.34 \pm 0.03$ & $0.80 \pm 0.09$ & $<0.18$ & $0.41 \pm 0.12$ & $<0.22$ & $<0.22$ & $<0.24$ & $<0.20$ \\
\hline \multicolumn{11}{|c|}{ RO effluent: Free Chlorine } \\
\hline Event 1 & $12.2 \pm 1.1$ & $<0.20$ & $0.98 \pm 0.17$ & $1.4 \pm 0.2$ & $<0.18$ & $3.0 \pm 0.9$ & $<0.22$ & $<0.22$ & $<0.24$ & $<0.20$ \\
\hline Event 2 & $3.6 \pm 0.2$ & $<0.20$ & $1.2 \pm 0.1$ & $2.5 \pm 0.4$ & $<0.18$ & $1.9 \pm 0.5$ & $<0.22$ & $<0.22$ & $<0.24$ & $<0.20$ \\
\hline Event 3 & $8.6 \pm 0.3$ & $<0.20$ & $1.9 \pm 0.4$ & $3.4 \pm 0.4$ & $<0.18$ & $3.1 \pm 0.3$ & $<0.22$ & $<0.22$ & $<0.24$ & $<0.20$ \\
\hline Event $420 \%$ Processed & $2.8 \pm 0.2$ & $<0.20$ & $1.2 \pm 0.2$ & $1.2 \pm 0.1$ & $<0.18$ & $2.8 \pm 0.4$ & $<0.22$ & $<0.22$ & $<0.24$ & $<0.20$ \\
\hline Event $450 \%$ Processed & $4.9 \pm 1.4$ & $<0.20$ & $1.0 \pm 0.3$ & $1.0 \pm 0.1$ & $<0.18$ & $1.1 \pm 0.4$ & $<0.22$ & $<0.22$ & $<0.24$ & $<0.20$ \\
\hline Event $490 \%$ Processed & $3.5 \pm 1.1$ & $<0.20$ & $2.2 \pm 0.3$ & $3.4 \pm 0.2$ & $<0.18$ & $4.6 \pm 1.4$ & $<0.22$ & $<0.22$ & $<0.24$ & $<0.20$ \\
\hline MRL* & 0.22 & 0.20 & 0.17 & 0.16 & 0.18 & 0.18 & 0.22 & 0.22 & 0.24 & 0.20 \\
\hline
\end{tabular}


Table S15. THM4 detected in samples collected during decentralized treatment of greywater.

\begin{tabular}{|c|c|c|c|c|}
\hline Sample & TCM & BDCM & DBCM & TBM \\
\hline \hline \multicolumn{5}{|c|}{ Feed } \\
\hline Event 1 & $8.2 \pm 0.8$ & $0.47 \pm 0.02$ & $0.41 \pm 0.02$ & $0.21 \pm 0.01$ \\
Event 2 & $40.3 \pm 9.6$ & $0.78 \pm 0.18$ & $0.79 \pm 0.14$ & $0.49 \pm 0.06$ \\
Event 3 & $185.7 \pm 12.6$ & $<0.18$ & $0.28 \pm 0.01$ & $0.23 \pm 0.03$ \\
Event 4 20\% Processed & $174.5 \pm 25.6$ & $<0.18$ & $0.51 \pm 0.08$ & $0.29 \pm 0.05$ \\
Event 4 50\% Processed & $113.9 \pm 21.9$ & $<0.18$ & $0.41 \pm 0.05$ & $0.26 \pm 0.05$ \\
Event 4 90\% Processed & $313.9 \pm 10.0$ & $<0.18$ & $0.74 \pm 0.02$ & $0.39 \pm 0.04$ \\
\hline \multicolumn{5}{|c|}{ Between FO and RO } \\
\hline Event 1 & $987.4 \pm 100.8$ & $0.19 \pm 0.02$ & $<0.16$ & $<0.21$ \\
Event 2 & $484.6 \pm 35.9$ & $<0.18$ & $0.24 \pm 0.05$ & $<0.21$ \\
Event 3 & $974.4 \pm 27.2$ & $<0.18$ & $0.28 \pm 0.01$ & $0.21 \pm 0.02$ \\
Event 4 20\% Processed & $902.4 \pm 66.4$ & $0.31 \pm 0.01$ & $0.16 \pm 0.01$ & $<0.21$ \\
Event 4 50\% Processed & $716.2 \pm 62.5$ & $0.25 \pm 0.04$ & $0.16 \pm 0.01$ & $<0.21$ \\
Event 4 90\% Processed & $640.5 \pm 23.1$ & $0.29 \pm 0.10$ & $0.16 \pm 0.01$ & $<0.21$ \\
\hline \multicolumn{5}{|c|}{ RO effluent } \\
\hline Event 1 & $46.8 \pm 1.5$ & $<0.18$ & $<0.16$ & $<0.21$ \\
Event 2 & $15.6 \pm 1.4$ & $<0.18$ & $<0.16$ & $<0.21$ \\
Event 3 & $39.1 \pm 4.8$ & $<0.18$ & $<0.16$ & $<0.21$ \\
Event 4 20\% Processed & $50.3 \pm 1.5$ & $<0.18$ & $<0.16$ & $<0.21$ \\
Event 4 50\% Processed & $42.3 \pm 1.8$ & $<0.18$ & $<0.16$ & $<0.21$ \\
Event 4 90\% Processed & $31.6 \pm 4.5$ & $<0.18$ & $<0.16$ & $<0.21$ \\
\hline \hline MRL* & 0.24 & 0.18 & 0.16 & 0.21 \\
\hline
\end{tabular}


Table S16. THM4 detected after chloramine or chlorine treatment in samples collected during decentralized treatment of greywater.

\begin{tabular}{|c|c|c|c|c|}
\hline Sample & TCM & BDCM & DBCM & TBM \\
\hline \multicolumn{5}{|c|}{ Feed: Chloramines } \\
\hline Event 1 & $8.3 \pm 0.3$ & $0.65 \pm 0.02$ & $0.30 \pm 0.02$ & $0.21 \pm 0.01$ \\
\hline Event 2 & $47.3 \pm 0.6$ & $0.73 \pm 0.07$ & $0.53 \pm 0.01$ & $0.35 \pm 0.08$ \\
\hline Event 3 & $184.1 \pm 36.3$ & $0.32 \pm 0.04$ & $0.19 \pm 0.04$ & $0.21 \pm 0.01$ \\
\hline Event $420 \%$ Processed & $174.6 \pm 14.8$ & $1.1 \pm 0.1$ & $0.45 \pm 0.01$ & $0.29 \pm 0.03$ \\
\hline Event $450 \%$ Processed & $198.6 \pm 1.3$ & $1.5 \pm 0.1$ & $0.48 \pm 0.03$ & $0.30 \pm 0.05$ \\
\hline Event $490 \%$ Processed & $336.9 \pm 37.3$ & $1.4 \pm 0.1$ & $0.47 \pm 0.01$ & $0.25 \pm 0.01$ \\
\hline \multicolumn{5}{|c|}{ Between FO and RO: Chloramines } \\
\hline Event 1 & $928.9 \pm 8.5$ & $0.18 \pm 0.01$ & $<0.16$ & $<0.21$ \\
\hline Event 2 & $295.1 \pm 41.5$ & $0.37 \pm 0.01$ & $0.20 \pm 0.01$ & $<0.21$ \\
\hline Event 3 & $689.5 \pm 26.7$ & $0.23 \pm 0.02$ & $0.19 \pm 0.01$ & $<0.21$ \\
\hline Event $420 \%$ Processed & $586.2 \pm 25.1$ & $0.21 \pm 0.01$ & $0.16 \pm 0.01$ & $<0.21$ \\
\hline Event $450 \%$ Processed & $531.9 \pm 61.9$ & $0.21 \pm 0.01$ & $0.16 \pm 0.01$ & $<0.21$ \\
\hline Event $490 \%$ Processed & $540.0 \pm 60.2$ & $0.25 \pm 0.02$ & $0.16 \pm 0.01$ & $<0.21$ \\
\hline \multicolumn{5}{|c|}{ RO effluent: Chloramines } \\
\hline Event 1 & $50.7 \pm 6.6$ & $<0.18$ & $<0.16$ & $<0.21$ \\
\hline Event 2 & $22.1 \pm 4.8$ & $<0.18$ & $<0.16$ & $<0.21$ \\
\hline Event 3 & $39.9 \pm 6.8$ & $<0.18$ & $<0.16$ & $<0.21$ \\
\hline Event $420 \%$ Processed & $54.1 \pm 4.5$ & $<0.18$ & $<0.16$ & $<0.21$ \\
\hline Event $450 \%$ Processed & $47.6 \pm 6.2$ & $<0.18$ & $<0.16$ & $<0.21$ \\
\hline Event $490 \%$ Processed & $48.6 \pm 2.5$ & $0.29 \pm 0.04$ & $<0.16$ & $<0.21$ \\
\hline \multicolumn{5}{|c|}{ RO effluent: Free Chlorine } \\
\hline Event 1 & $48.0 \pm 4.6$ & $1.1 \pm 0.1$ & $<0.16$ & $<0.21$ \\
\hline Event 2 & $42.3 \pm 2.6$ & $0.20 \pm 0.02$ & $<0.16$ & $<0.21$ \\
\hline Event 3 & $90.4 \pm 5.1$ & $1.8 \pm 0.2$ & $<0.16$ & $<0.21$ \\
\hline Event $420 \%$ Processed & $38.1 \pm 3.3$ & $1.2 \pm 0.1$ & $0.17 \pm 0.01$ & $<0.21$ \\
\hline Event $450 \%$ Processed & $90.8 \pm 10.2$ & $0.41 \pm 0.06$ & $<0.16$ & $<0.21$ \\
\hline Event $490 \%$ Processed & $77.3 \pm 4.7$ & $0.62 \pm 0.04$ & $<0.16$ & $<0.21$ \\
\hline MRL* & 0.24 & 0.18 & 0.16 & 0.21 \\
\hline
\end{tabular}


Table S17. THM4 detected in samples collected during decentralized treatment of sewage.

\begin{tabular}{|c|c|c|c|c|}
\hline Sample & TCM & BDCM & DBCM & TBM \\
\hline \hline \multicolumn{5}{|c|}{ Feed } \\
\hline Event 1 & $0.94 \pm 0.11$ & $0.39 \pm 0.04$ & $<0.16$ & $<0.21$ \\
Event 2 & $1.5 \pm 0.1$ & $0.37 \pm 0.02$ & $<0.16$ & $<0.21$ \\
Event 3 & $1.7 \pm 0.2$ & $0.36 \pm 0.03$ & $<0.16$ & $<0.21$ \\
Event 4 20\% Processed & $1.0 \pm 0.1$ & $0.31 \pm 0.08$ & $<0.16$ & $<0.21$ \\
Event 4 50\% Processed & $1.4 \pm 0.1$ & $0.37 \pm 0.03$ & $<0.16$ & $<0.21$ \\
Event 4 90\% Processed & $2.1 \pm 0.1$ & $0.21 \pm 0.02$ & $<0.16$ & $<0.21$ \\
\hline \multicolumn{5}{|c|}{ Between FO and RO } \\
\hline Event 1 & $10.9 \pm 2.2$ & $0.36 \pm 0.02$ & $0.16 \pm 0.01$ & $0.21 \pm 0.03$ \\
Event 2 & $8.7 \pm 0.8$ & $0.27 \pm 0.03$ & $<0.16$ & $<0.21$ \\
Event 3 & $8.3 \pm 0.5$ & $0.23 \pm 0.04$ & $0.16 \pm 0.01$ & $<0.21$ \\
Event 4 20\% Processed & $3.8 \pm 0.1$ & $0.32 \pm 0.02$ & $<0.16$ & $<0.21$ \\
Event 4 50\% Processed & $4.4 \pm 0.3$ & $0.24 \pm 0.01$ & $<0.16$ & $<0.21$ \\
Event 4 90\% Processed & $3.4 \pm 0.1$ & $0.27 \pm 0.04$ & $<0.16$ & $<0.21$ \\
\hline \multicolumn{5}{|c|}{ RO effluent } \\
\hline Event 1 & $0.25 \pm 0.01$ & $<0.18$ & $<0.16$ & $<0.21$ \\
Event 2 & $1.8 \pm 0.2$ & $<0.18$ & $<0.16$ & $<0.21$ \\
Event 3 & $1.6 \pm 0.1$ & $<0.18$ & $<0.16$ & $<0.21$ \\
Event 4 20\% Processed & $1.7 \pm 0.3$ & $<0.18$ & $<0.16$ & $<0.21$ \\
Event 4 50\% Processed & $1.7 \pm 0.1$ & $<0.18$ & $<0.16$ & $<0.21$ \\
Event 4 90\% Processed & $1.6 \pm 0.1$ & $<0.18$ & $<0.16$ & $<0.21$ \\
\hline \hline \multicolumn{5}{|c|}{ MRL* }
\end{tabular}


Table S18. THM4 detected after chloramine or chlorine treatment in samples collected during decentralized treatment of sewage.

\begin{tabular}{|c|c|c|c|c|}
\hline Sample & TCM & BDCM & DBCM & TBM \\
\hline \hline \multicolumn{5}{|c|}{ Feed: Chloramines } \\
\hline Event 1 & $5.3 \pm 0.8$ & $0.38 \pm 0.05$ & $<0.16$ & $<0.21$ \\
Event 2 & $3.4 \pm 0.4$ & $0.30 \pm 0.04$ & $<0.16$ & $<0.21$ \\
Event 3 & $6.0 \pm 0.7$ & $0.36 \pm 0.09$ & $<0.16$ & $<0.21$ \\
Event 4 20\% Processed & $5.0 \pm 0.4$ & $0.38 \pm 0.10$ & $<0.16$ & $<0.21$ \\
Event 4 50\% Processed & $2.6 \pm 0.2$ & $0.38 \pm 0.05$ & $<0.16$ & $<0.21$ \\
Event 4 90\% Processed & $11.8 \pm 2.0$ & $0.32 \pm 0.02$ & $<0.16$ & $<0.21$ \\
\hline \multicolumn{5}{|c|}{ Between FO and RO: Chloramines } \\
\hline Event 1 & $16.1 \pm 2.1$ & $0.19 \pm 0.01$ & $0.25 \pm 0.02$ & $0.21 \pm 0.01$ \\
Event 2 & $13.9 \pm 0.9$ & $0.21 \pm 0.02$ & $0.35 \pm 0.03$ & $0.23 \pm 0.07$ \\
Event 3 & $11.6 \pm 1.1$ & $0.20 \pm 0.04$ & $0.24 \pm 0.02$ & $0.21 \pm 0.02$ \\
Event 4 20\% Processed & $7.1 \pm 0.3$ & $0.19 \pm 0.01$ & $0.21 \pm 0.02$ & $0.21 \pm 0.01$ \\
Event 4 50\% Processed & $7.7 \pm 0.6$ & $0.25 \pm 0.01$ & $<0.16$ & $<0.21 \pm 0.01$ \\
Event 4 90\% Processed & $5.2 \pm 0.5$ & $0.27 \pm 0.07$ & $<0.16$ & $<0.21$ \\
\hline \multicolumn{5}{|c|}{ RO effluent: Chloramines } \\
\hline Event 1 & $1.3 \pm 0.4$ & $0.25 \pm 0.01$ & $<0.16$ & $<0.21$ \\
Event 2 & $1.2 \pm 0.4$ & $<0.18$ & $<0.16$ & $<0.21$ \\
Event 3 & $1.3 \pm 0.3$ & $<0.18$ & $<0.16$ & $<0.21$ \\
Event 4 20\% Processed & $1.8 \pm 0.2$ & $0.35 \pm 0.04$ & $0.21 \pm 0.04$ & $<0.21$ \\
Event 4 50\% Processed & $1.7 \pm 0.2$ & $0.18 \pm 0.01$ & $<0.16$ & $<0.21$ \\
Event 4 90\% Processed & $1.8 \pm 0.1$ & $0.33 \pm 0.03$ & $0.18 \pm 0.03$ & $<0.21$ \\
\hline \hline MvL* & 0.24 & 0.18 & 0.16 & 0.21 \\
\hline Event 4 20\% Processed & $1.2 \pm 0.4$ & $<0.18$ & $<0.16$ & $<0.21$ \\
Event 4 50\% Processed & $1.8 \pm 0.1$ & $<0.18$ & $<0.16$ & $<0.21$ \\
Event 4 90\% Processed & $1.8 \pm 0.1$ & $<0.18$ & $<0.16$ & $<0.21$ \\
\hline \multicolumn{5}{|c|}{ RO effluent: Free Chlorine } \\
\hline Event 1 & $1.4 \pm 0.2$ & $0.27 \pm 0.03$ & $<0.16$ & $<0.21$ \\
Event 2 & $1.5 \pm 0.2$ & $0.34 \pm 0.08$ & $<0.16$ & $<0.21$ \\
\hline
\end{tabular}


Table S19. HAMs detected in samples collected during decentralized treatment of greywater.

\begin{tabular}{|c|c|c|c|c|}
\hline Sample & TCAM & DCAM & BCAM & DBAM \\
\hline \hline \multicolumn{5}{|c|}{ Feed } \\
\hline Event 1 & $<0.24$ & $3.7 \pm 0.6$ & $<0.17$ & $<0.22$ \\
Event 2 & $<0.24$ & $9.5 \pm 0.3$ & $0.27 \pm 0.05$ & $<0.22$ \\
Event 3 & $<0.24$ & $12.2 \pm 0.6$ & $<0.17$ & $<0.22$ \\
Event 4 20\% Processed & $<0.24$ & $5.5 \pm 0.2$ & $<0.17$ & $<0.22$ \\
Event 4 50\% Processed & $<0.24$ & $5.0 \pm 0.1$ & $<0.17$ & $<0.22$ \\
Event 4 90\% Processed & $<0.24$ & $7.1 \pm 2.2$ & $<0.17$ & $<0.22$ \\
\hline \multicolumn{5}{|c|}{ Between FO and RO } \\
\hline Event 1 & $<0.24$ & $3.7 \pm 0.2$ & $<0.17$ & $<0.22$ \\
Event 2 & $<0.24$ & $9.0 \pm 0.7$ & $<0.17$ & $<0.22$ \\
Event 3 & $<0.24$ & $11.3 \pm 0.3$ & $0.17 \pm 0.01$ & $<0.22$ \\
Event 4 20\% Processed & $<0.24$ & $5.2 \pm 0.6$ & $<0.17$ & $<0.22$ \\
Event 4 50\% Processed & $<0.24$ & $4.1 \pm 0.6$ & $<0.17$ & $<0.22$ \\
Event 4 90\% Processed & $<0.24$ & $6.1 \pm 0.5$ & $<0.17$ & $<0.22$ \\
\hline \multicolumn{5}{|c|}{ RO effluent } \\
\hline Event 1 & $<0.24$ & $<0.14$ & $<0.17$ & $<0.22$ \\
Event 2 & $<0.24$ & $<0.14$ & $<0.17$ & $<0.22$ \\
Event 3 & $<0.24$ & $0.35 \pm 0.03$ & $<0.17$ & $<0.22$ \\
Event 4 20\% Processed & $<0.24$ & $<0.31 \pm 0.02$ & $<0.17$ & $<0.22$ \\
Event 4 50\% Processed & $<0.24$ & $<0.14$ & $<0.17$ & $<0.22$ \\
Event 4 90\% Processed & $<0.24$ & $<0.14$ & $<0.17$ & $<0.22$ \\
\hline \hline \multicolumn{5}{|c|}{ MRL* }
\end{tabular}


Table S20. HAMs detected after chloramine or chlorine treatment in samples collected during decentralized treatment of greywater.

\begin{tabular}{|c|c|c|c|c|}
\hline Sample & TCAM & DCAM & BCAM & DBAM \\
\hline \multicolumn{5}{|c|}{ Feed: Chloramines } \\
\hline Event 1 & $<0.24$ & $11.6 \pm 1.4$ & $<0.17$ & $<0.22$ \\
\hline Event 2 & $<0.24$ & $14.4 \pm 0.6$ & $<0.17$ & $<0.22$ \\
\hline Event 3 & $<0.24$ & $8.7 \pm 1.5$ & $<0.17$ & $<0.22$ \\
\hline Event $420 \%$ Processed & $<0.24$ & $10.1 \pm 1.4$ & $<0.17$ & $<0.22$ \\
\hline Event $450 \%$ Processed & $<0.24$ & $24.5 \pm 2.9$ & $<0.17$ & $<0.22$ \\
\hline Event $490 \%$ Processed & $<0.24$ & $19.9 \pm 3.2$ & $<0.17$ & $<0.22$ \\
\hline \multicolumn{5}{|c|}{ Between FO and RO: Chloramines } \\
\hline Event 1 & $<0.24$ & $4.2 \pm 0.8$ & $0.21 \pm 0.03$ & $<0.22$ \\
\hline Event 2 & $<0.24$ & $10.9 \pm 0.7$ & $0.31 \pm 0.03$ & $<0.22$ \\
\hline Event 3 & $<0.24$ & $14.8 \pm 0.5$ & $<0.17$ & $<0.22$ \\
\hline Event $420 \%$ Processed & $<0.24$ & $6.8 \pm 0.2$ & $<0.17$ & $<0.22$ \\
\hline Event $450 \%$ Processed & $<0.24$ & $7.4 \pm 0.3$ & $<0.17$ & $<0.22$ \\
\hline Event $490 \%$ Processed & $<0.24$ & $8.5 \pm 0.5$ & $<0.17$ & $<0.22$ \\
\hline \multicolumn{5}{|c|}{ RO effluent: Chloramines } \\
\hline Event 1 & $<0.24$ & $<0.14$ & $<0.17$ & $<0.22$ \\
\hline Event 2 & $<0.24$ & $0.15 \pm 0.02$ & $<0.17$ & $<0.22$ \\
\hline Event 3 & $<0.24$ & $0.73 \pm 0.06$ & $<0.17$ & $<0.22$ \\
\hline Event $420 \%$ Processed & $<0.24$ & $0.33 \pm 0.04$ & $<0.17$ & $<0.22$ \\
\hline Event $450 \%$ Processed & $<0.24$ & $0.31 \pm 0.03$ & $<0.17$ & $<0.22$ \\
\hline Event $490 \%$ Processed & $<0.24$ & $0.56 \pm 0.04$ & $<0.17$ & $<0.22$ \\
\hline \multicolumn{5}{|c|}{ RO effluent: Free Chlorine } \\
\hline Event 1 & $<0.24$ & $<0.14$ & $<0.17$ & $<0.22$ \\
\hline Event 2 & $<0.24$ & $<0.14$ & $<0.17$ & $<0.22$ \\
\hline Event 3 & $<0.24$ & $<0.14$ & $<0.17$ & $<0.22$ \\
\hline Event $420 \%$ Processed & $<0.24$ & $0.35 \pm 0.08$ & $<0.17$ & $<0.22$ \\
\hline Event $450 \%$ Processed & $<0.24$ & $0.36 \pm 0.03$ & $<0.17$ & $<0.22$ \\
\hline Event $490 \%$ Processed & $<0.24$ & $<0.14$ & $<0.17$ & $<0.22$ \\
\hline MRL* & 0.24 & 0.14 & 0.17 & 0.22 \\
\hline
\end{tabular}


Table S21. HAMs detected in samples collected during decentralized treatment of sewage.

\begin{tabular}{|c|c|c|c|c|}
\hline Sample & TCAM & DCAM & BCAM & DBAM \\
\hline \hline \multicolumn{5}{|c|}{ Feed } \\
\hline Event 1 & $0.28 \pm 0.02$ & $30.2 \pm 7.9$ & $0.59 \pm 0.24$ & $1.8 \pm 0.7$ \\
Event 2 & $<0.24$ & $5.7 \pm 0.4$ & $0.70 \pm 0.19$ & $4.4 \pm 0.2$ \\
Event 3 & $<0.24$ & $24.1 \pm 1.5$ & $0.30 \pm 0.02$ & $9.1 \pm 1.2$ \\
Event 4 20\% Processed & $<0.24$ & $7.4 \pm 0.2$ & $0.32 \pm 0.08$ & $2.2 \pm 0.6$ \\
Event 4 50\% Processed & $0.55 \pm 0.10$ & $13.3 \pm 1.7$ & $0.17 \pm 0.01$ & $1.6 \pm 0.1$ \\
Event 4 90\% Processed & $<0.24$ & $8.0 \pm 2.0$ & $<0.17$ & $0.72 \pm 0.26$ \\
\hline \multicolumn{5}{|c|}{ Between FO and RO } \\
\hline Event 1 & $<0.24$ & $9.5 \pm 1.5$ & $1.3 \pm 0.2$ & $0.22 \pm 0.01$ \\
Event 2 & $<0.24$ & $6.0 \pm 1.1$ & $0.73 \pm 0.15$ & $0.22 \pm 0.01$ \\
Event 3 & $<0.24$ & $6.5 \pm 1.6$ & $0.77 \pm 0.15$ & $<0.22$ \\
Event 4 20\% Processed & $<0.24$ & $5.9 \pm 0.8$ & $0.46 \pm 0.03$ & $<0.22$ \\
Event 4 50\% Processed & $<0.24$ & $4.1 \pm 1.6$ & $0.31 \pm 0.03$ & $<0.22$ \\
Event 4 90\% Processed & $<0.24$ & $6.2 \pm 0.8$ & $0.43 \pm 0.04$ & $<0.22$ \\
\hline \multicolumn{5}{|c|}{ RO effluent } \\
\hline Event 1 & $0.28 \pm 0.03$ & $30.2 \pm 0.8$ & $0.50 \pm 0.04$ & $1.8 \pm 0.6$ \\
Event 2 & $<0.24$ & $5.8 \pm 0.4$ & $0.70 \pm 0.19$ & $4.5 \pm 0.2$ \\
Event 3 & $<0.24$ & $24.1 \pm 1.5$ & $0.30 \pm 0.02$ & $9.1 \pm 1.2$ \\
Event 4 20\% Processed & $<0.24$ & $7.4 \pm 2.3$ & $0.32 \pm 0.7$ & $2.2 \pm 0.6$ \\
Event 4 50\% Processed & $0.55 \pm 0.25$ & $13.3 \pm 1.6$ & $0.17 \pm 0.01$ & $1.6 \pm 0.1$ \\
Event 4 90\% Processed & $<0.24$ & $8.1 \pm 1.9$ & $0.17 \pm 0.01$ & $0.72 \pm 0.26$ \\
\hline \hline \multicolumn{5}{|c|}{ MRL* }
\end{tabular}


Table S22. Lab scale FO membrane experiment descriptions. HAMs detected after chloramine or chlorine treatment in samples collected during decentralized treatment of sewage.

\begin{tabular}{|c|c|c|c|c|}
\hline Sample & TCAM & DCAM & BCAM & DBAM \\
\hline \multicolumn{5}{|c|}{ Feed: Chloramines } \\
\hline Event 1 & $2.2 \pm 0.3$ & $149.7 \pm 25.4$ & $0.64 \pm 0.20$ & $4.1 \pm 1.3$ \\
\hline Event 2 & $1.5 \pm 0.3$ & $144.3 \pm 2.5$ & $0.93 \pm 0.16$ & $0.61 \pm 0.14$ \\
\hline Event 3 & $0.32 \pm 0.04$ & $50.7 \pm 6.4$ & $0.43 \pm 0.11$ & $1.4 \pm 0.2$ \\
\hline Event $420 \%$ Processed & $<0.24$ & $58.2 \pm 1.9$ & $0.41 \pm 0.12$ & $1.1 \pm 0.01$ \\
\hline Event $450 \%$ Processed & $0.99 \pm 0.24$ & $19.5 \pm 3.7$ & $0.49 \pm 0.09$ & $1.5 \pm 0.4$ \\
\hline Event $490 \%$ Processed & $2.1 \pm 0.5$ & $154.0 \pm 15.5$ & $0.45 \pm 0.13$ & $9.3 \pm 0.7$ \\
\hline \multicolumn{5}{|c|}{ Between FO and RO: Chloramines } \\
\hline Event 1 & $<0.24$ & $12.2 \pm 1.0$ & $1.5 \pm 0.2$ & $0.36 \pm 0.05$ \\
\hline Event 2 & $<0.24$ & $15.7 \pm 2.6$ & $0.87 \pm 0.04$ & $<0.22$ \\
\hline Event 3 & $<0.24$ & $6.8 \pm 0.6$ & $0.78 \pm 0.07$ & $0.26 \pm 0.08$ \\
\hline Event $420 \%$ Processed & $<0.24$ & $13.2 \pm 1.4$ & $0.89 \pm 0.06$ & $<0.22$ \\
\hline Event $450 \%$ Processed & $<0.24$ & $19.2 \pm 3.7$ & $1.6 \pm 0.2$ & $0.26 \pm 0.04$ \\
\hline Event $490 \%$ Processed & $<0.24$ & $20.3 \pm 1.7$ & $3.0 \pm 0.1$ & $0.39 \pm 0.02$ \\
\hline \multicolumn{5}{|c|}{ RO effluent: Chloramines } \\
\hline Event 1 & $<0.24$ & $<0.14$ & $<0.17$ & $<0.22$ \\
\hline Event 2 & $<0.24$ & $<0.14$ & $<0.17$ & $<0.22$ \\
\hline Event 3 & $<0.24$ & $<0.14$ & $<0.17$ & $<0.22$ \\
\hline Event $420 \%$ Processed & $<0.24$ & $0.15 \pm 0.01$ & $<0.17$ & $<0.22$ \\
\hline Event $450 \%$ Processed & $<0.24$ & $<0.14$ & $<0.17$ & $<0.22$ \\
\hline Event $490 \%$ Processed & $<0.24$ & $0.16 \pm 0.03$ & $<0.17$ & $<0.22$ \\
\hline \multicolumn{5}{|c|}{ RO effluent: Free Chlorine } \\
\hline Event 1 & $<0.24$ & $0.16 \pm 0.03$ & $<0.17$ & $<0.22$ \\
\hline Event 2 & $<0.24$ & $<0.14$ & $<0.17$ & $<0.22$ \\
\hline Event 3 & $<0.24$ & $<0.14$ & $<0.17$ & $<0.22$ \\
\hline Event $420 \%$ Processed & $<0.24$ & $<0.14$ & $<0.17$ & $<0.22$ \\
\hline Event $450 \%$ Processed & $<0.24$ & $<0.14$ & $<0.17$ & $<0.22$ \\
\hline Event $490 \%$ Processed & $<0.24$ & $<0.14$ & $<0.17$ & $<0.22$ \\
\hline MRL* & 0.24 & 0.14 & 0.17 & 0.22 \\
\hline
\end{tabular}


Table S23. HALs detected in samples collected during decentralized treatment of greywater.

\begin{tabular}{|c|c|c|c|c|}
\hline Sample & TCAL & BDCAL & DBCAL & TBAL \\
\hline \hline \multicolumn{5}{|c|}{ Feed } \\
\hline Event 1 & $2.1 \pm 0.1$ & $<0.14$ & $0.88 \pm 0.13$ & $<0.33$ \\
Event 2 & $10.7 \pm 3.1$ & $<0.14$ & $0.66 \pm 0.11$ & $<0.33$ \\
Event 3 & $0.50 \pm 0.04$ & $<0.14$ & $1.5 \pm 0.6$ & $<0.33$ \\
Event 4 20\% Processed & $1.1 \pm 0.3$ & $<0.14$ & $0.72 \pm 0.07$ & $<0.33$ \\
Event 4 50\% Processed & $0.73 \pm 0.12$ & $<0.14$ & $0.71 \pm 0.05$ & $<0.33$ \\
Event 4 90\% Processed & $1.6 \pm 0.1$ & $<0.14$ & $1.0 \pm 0.2$ & $<0.33$ \\
\hline \multicolumn{5}{|c|}{ Between FO and RO } \\
\hline Event 1 & $0.27 \pm 0.01$ & $<0.14$ & $<0.20$ & $<0.33$ \\
Event 2 & $0.70 \pm 0.17$ & $<0.14$ & $<0.20$ & $<0.33$ \\
Event 3 & $0.69 \pm 0.01$ & $<0.14$ & $<0.20$ & $<0.33$ \\
Event 4 20\% Processed & $0.36 \pm 0.01$ & $<0.14$ & $<0.20$ & $<0.33$ \\
Event 4 50\% Processed & $0.37 \pm 0.01$ & $<0.14$ & $<0.20$ & $<0.33$ \\
Event 4 90\% Processed & $0.39 \pm 0.03$ & $<0.14$ & $<0.20$ & $<0.33$ \\
\hline \multicolumn{5}{|c|}{ RO effluent } \\
\hline Event 1 & $<0.15$ & $<0.14$ & $<0.20$ & $<0.33$ \\
Event 2 & $<0.15$ & $<0.14$ & $<0.20$ & $<0.33$ \\
Event 3 & $3.2 \pm 0.5$ & $<0.14$ & $<0.20$ & $<0.33$ \\
Event 4 20\% Processed & $<0.15$ & $<0.14$ & $<0.20$ & $<0.33$ \\
Event 4 50\% Processed & $0.69 \pm 0.21$ & $<0.14$ & $<0.20$ & $<0.33$ \\
Event 4 90\% Processed & $0.21 \pm 0.04$ & $<0.14$ & $<0.20$ & $<0.33$ \\
\hline \hline \multicolumn{5}{|c|}{ MRL* }
\end{tabular}


Table S24. HALs detected after chloramine or chlorine treatment in samples collected during decentralized treatment of greywater.

\begin{tabular}{|c|c|c|c|c|}
\hline Sample & TCAL & BDCAL & DBCAL & TBAL \\
\hline \multicolumn{5}{|c|}{ Feed: Chloramines } \\
\hline Event 1 & $3.7 \pm 0.4$ & $<0.14$ & $0.94 \pm 0.10$ & $<0.33$ \\
\hline Event 2 & $7.3 \pm 2.3$ & $<0.14$ & $0.97 \pm 0.17$ & $<0.33$ \\
\hline Event 3 & $1.4 \pm 0.1$ & $<0.14$ & $0.94 \pm 0.24$ & $<0.33$ \\
\hline Event $420 \%$ Processed & $6.9 \pm 1.1$ & $<0.14$ & $1.1 \pm 0.2$ & $<0.33$ \\
\hline Event $450 \%$ Processed & $8.1 \pm 1.0$ & $<0.14$ & $1.5 \pm 0.5$ & $<0.33$ \\
\hline Event $490 \%$ Processed & $6.7 \pm 1.1$ & $<0.14$ & $1.1 \pm 0.1$ & $<0.33$ \\
\hline \multicolumn{5}{|c|}{ Between FO and RO: Chloramines } \\
\hline Event 1 & $0.32 \pm 0.03$ & $<0.14$ & $0.68 \pm 0.13$ & $<0.33$ \\
\hline Event 2 & $1.3 \pm 0.1$ & $<0.14$ & $0.55 \pm 0.07$ & $<0.33$ \\
\hline Event 3 & $0.71 \pm 0.05$ & $<0.14$ & $0.48 \pm 0.11$ & $<0.33$ \\
\hline Event $420 \%$ Processed & $0.43 \pm 0.04$ & $<0.14$ & $0.57 \pm 0.02$ & $<0.33$ \\
\hline Event $450 \%$ Processed & $0.42 \pm 0.02$ & $<0.14$ & $0.59 \pm 0.07$ & $<0.33$ \\
\hline Event $490 \%$ Processed & $0.46 \pm 0.03$ & $<0.14$ & $0.51 \pm 0.07$ & $<0.33$ \\
\hline \multicolumn{5}{|c|}{ RO effluent: Chloramines } \\
\hline Event 1 & $<0.15$ & $<0.14$ & $0.48 \pm 0.04$ & $<0.33$ \\
\hline Event 2 & $4.0 \pm 0.5$ & $<0.14$ & $0.52 \pm 0.01$ & $<0.33$ \\
\hline Event 3 & $6.5 \pm 1.5$ & $<0.14$ & $0.45 \pm 0.06$ & $<0.33$ \\
\hline Event $420 \%$ Processed & $<0.15$ & $<0.14$ & $0.59 \pm 0.09$ & $<0.33$ \\
\hline Event $450 \%$ Processed & $1.7 \pm 0.1$ & $<0.14$ & $0.56 \pm 0.02$ & $<0.33$ \\
\hline Event $490 \%$ Processed & $2.7 \pm 0.1$ & $<0.14$ & $0.52 \pm 0.03$ & $<0.33$ \\
\hline \multicolumn{5}{|c|}{ RO effluent: Free Chlorine } \\
\hline Event 1 & $1.3 \pm 0.1$ & $<0.14$ & $0.41 \pm 0.06$ & $<0.33$ \\
\hline Event 2 & $5.5 \pm 1.7$ & $<0.14$ & $0.41 \pm 0.03$ & $<0.33$ \\
\hline Event 3 & $8.5 \pm 2.1$ & $<0.14$ & $0.51 \pm 0.03$ & $<0.33$ \\
\hline Event $420 \%$ Processed & $9.1 \pm 3.1$ & $<0.14$ & $0.43 \pm 0.02$ & $<0.33$ \\
\hline Event $450 \%$ Processed & $2.0 \pm 0.1$ & $<0.14$ & $0.37 \pm 0.04$ & $<0.33$ \\
\hline Event $490 \%$ Processed & $2.7 \pm 0.6$ & $<0.14$ & $0.39 \pm 0.11$ & $<0.33$ \\
\hline MRL* & 0.15 & 0.14 & 0.20 & 0.33 \\
\hline
\end{tabular}


Table S25. HALs detected in samples collected during decentralized treatment of sewage.

\begin{tabular}{|c|c|c|c|c|}
\hline Sample & TCAL & BDCAL & DBCAL & TBAL \\
\hline \multicolumn{5}{|c|}{ Feed } \\
\hline Event 1 & $1.2 \pm 0.3$ & $<0.14$ & $<0.20$ & $<0.33$ \\
\hline Event 2 & $0.94 \pm 0.06$ & $<0.14$ & $<0.20$ & $<0.33$ \\
\hline Event 3 & $0.53 \pm 0.12$ & $<0.14$ & $<0.20$ & $<0.33$ \\
\hline Event $420 \%$ Processed & $0.49 \pm 0.18$ & $<0.14$ & $<0.20$ & $<0.33$ \\
\hline Event $450 \%$ Processed & $1.4 \pm 0.2$ & $<0.14$ & $<0.20$ & $<0.33$ \\
\hline Event $490 \%$ Processed & $0.42 \pm 0.07$ & $<0.14$ & $<0.20$ & $<0.33$ \\
\hline \multicolumn{5}{|c|}{ Between FO and RO } \\
\hline Event 1 & $0.24 \pm 0.05$ & $<0.14$ & $<0.20$ & $<0.33$ \\
\hline Event 2 & $0.33 \pm 0.03$ & $<0.14$ & $<0.20$ & $<0.33$ \\
\hline Event 3 & $0.34 \pm 0.03$ & $<0.14$ & $<0.20$ & $<0.33$ \\
\hline Event $420 \%$ Processed & $0.31 \pm 0.02$ & $<0.14$ & $<0.20$ & $<0.33$ \\
\hline Event $450 \%$ Processed & $0.32 \pm 0.06$ & $<0.14$ & $<0.20$ & $<0.33$ \\
\hline Event $490 \%$ Processed & $0.42 \pm 0.01$ & $<0.14$ & $<0.20$ & $<0.33$ \\
\hline \multicolumn{5}{|c|}{ RO effluent } \\
\hline Event 1 & $0.36 \pm 0.11$ & $<0.14$ & $<0.20$ & $<0.33$ \\
\hline Event 2 & $0.39 \pm 0.06$ & $<0.14$ & $<0.20$ & $<0.33$ \\
\hline Event 3 & $0.40 \pm 0.04$ & $<0.14$ & $<0.20$ & $<0.33$ \\
\hline Event $420 \%$ Processed & $0.51 \pm 0.10$ & $<0.14$ & $<0.20$ & $<0.33$ \\
\hline Event $450 \%$ Processed & $0.50 \pm 0.04$ & $<0.14$ & $<0.20$ & $<0.33$ \\
\hline Event $490 \%$ Processed & $0.29 \pm 0.02$ & $<0.14$ & $<0.20$ & $<0.33$ \\
\hline MRL* & 0.15 & 0.14 & 0.20 & 0.33 \\
\hline
\end{tabular}


Table S26. HALs detected after chloramine or chlorine treatment in samples collected during decentralized treatment of sewage.

\begin{tabular}{|c|c|c|c|c|}
\hline Sample & TCAL & BDCAL & DBCAL & TBAL \\
\hline \multicolumn{5}{|c|}{ Feed: Chloramines } \\
\hline Event 1 & $1.4 \pm 0.5$ & $<0.14$ & $<0.20$ & $<0.33$ \\
\hline Event 2 & $2.4 \pm 0.5$ & $<0.14$ & $<0.20$ & $<0.33$ \\
\hline Event 3 & $0.98 \pm 0.18$ & $<0.14$ & $<0.20$ & $<0.33$ \\
\hline Event $420 \%$ Processed & $0.78 \pm 0.24$ & $<0.14$ & $<0.20$ & $<0.33$ \\
\hline Event $450 \%$ Processed & $1.8 \pm 0.2$ & $<0.14$ & $<0.20$ & $<0.33$ \\
\hline Event $490 \%$ Processed & $1.1 \pm 0.1$ & $<0.14$ & $<0.20$ & $<0.33$ \\
\hline \multicolumn{5}{|c|}{ Between FO and RO: Chloramines } \\
\hline Event 1 & $0.40 \pm 0.06$ & $<0.14$ & $<0.20$ & $<0.33$ \\
\hline Event 2 & $0.44 \pm 0.04$ & $<0.14$ & $<0.20$ & $<0.33$ \\
\hline Event 3 & $0.38 \pm 0.08$ & $<0.14$ & $<0.20$ & $<0.33$ \\
\hline Event $420 \%$ Processed & $0.98 \pm 0.03$ & $<0.14$ & $<0.20$ & $<0.33$ \\
\hline Event $450 \%$ Processed & $0.60 \pm 0.10$ & $<0.14$ & $<0.20$ & $<0.33$ \\
\hline Event $490 \%$ Processed & $0.47 \pm 0.10$ & $<0.14$ & $<0.20$ & $<0.33$ \\
\hline \multicolumn{5}{|c|}{ RO effluent: Chloramines } \\
\hline Event 1 & $0.41 \pm 0.03$ & $<0.14$ & $<0.20$ & $<0.33$ \\
\hline Event 2 & $0.38 \pm 0.09$ & $<0.14$ & $<0.20$ & $<0.33$ \\
\hline Event 3 & $0.40 \pm 0.09$ & $<0.14$ & $<0.20$ & $<0.33$ \\
\hline Event $420 \%$ Processed & $0.51 \pm 0.02$ & $<0.14$ & $<0.20$ & $<0.33$ \\
\hline Event $450 \%$ Processed & $0.51 \pm 0.06$ & $<0.14$ & $<0.20$ & $<0.33$ \\
\hline Event $490 \%$ Processed & $0.43 \pm 0.11$ & $<0.14$ & $<0.20$ & $<0.33$ \\
\hline \multicolumn{5}{|c|}{ RO effluent: Free Chlorine } \\
\hline Event 1 & $2.1 \pm 0.1$ & $<0.14$ & $<0.20$ & $<0.33$ \\
\hline Event 2 & $1.3 \pm 0.3$ & $<0.14$ & $<0.20$ & $<0.33$ \\
\hline Event 3 & $1.3 \pm 0.2$ & $<0.14$ & $<0.20$ & $<0.33$ \\
\hline Event $420 \%$ Processed & $2.1 \pm 0.5$ & $<0.14$ & $<0.20$ & $<0.33$ \\
\hline Event $450 \%$ Processed & $0.84 \pm 0.13$ & $<0.14$ & $<0.20$ & $<0.33$ \\
\hline Event $490 \%$ Processed & $1.2 \pm 0.3$ & $<0.14$ & $<0.20$ & $<0.33$ \\
\hline MRL* & 0.15 & 0.14 & 0.20 & 0.33 \\
\hline
\end{tabular}


Table S27. HANs detected in samples collected during decentralized treatment of greywater.

\begin{tabular}{|c|c|c|c|c|}
\hline Sample & TCAN & DCAN & BCAN & DBAN \\
\hline \hline \multicolumn{5}{|c|}{ Feed } \\
\hline Event 1 & $<0.18$ & $0.14 \pm 0.01$ & $<0.16$ & $<0.21$ \\
Event 2 & $<0.18$ & $0.16 \pm 0.02$ & $<0.16$ & $<0.21$ \\
Event 3 & $<0.18$ & $<0.14$ & $<0.16$ & $<0.21$ \\
Event 4 20\% Processed & $<0.18$ & $0.14 \pm 0.01$ & $<0.16$ & $<0.21$ \\
Event 4 50\% Processed & $<0.18$ & $<0.14$ & $<0.16$ & $<0.21$ \\
Event 4 90\% Processed & $<0.18$ & $<0.14$ & $<0.16$ & $<0.21$ \\
\hline \multicolumn{5}{|c|}{ Between FO and RO } \\
\hline Event 1 & $<0.18$ & $<0.14$ & $<0.16$ & $<0.21$ \\
Event 2 & $<0.18$ & $<0.14$ & $<0.16$ & $<0.21$ \\
Event 3 & $<0.18$ & $<0.14$ & $<0.16$ & $<0.21$ \\
Event 4 20\% Processed & $<0.18$ & $<0.14$ & $<0.16$ & $<0.21$ \\
Event 4 50\% Processed & $<0.18$ & $<0.14$ & $<0.16$ & $<0.21$ \\
Event 4 90\% Processed & $<0.18$ & $<0.14$ & $<0.16$ & $<0.21$ \\
\hline \multicolumn{5}{|c|}{ RO effluent } \\
\hline Event 1 & $<0.18$ & $<0.14$ & $<0.16$ & $<0.21$ \\
Event 2 & $<0.18$ & $<0.14$ & $<0.16$ & $<0.21$ \\
Event 3 & $<0.18$ & $0.29 \pm 0.08$ & $<0.16$ & $<0.21$ \\
Event 4 20\% Processed & $<0.18$ & $<0.14$ & $<0.16$ & $<0.21$ \\
Event 4 50\% Processed & $<0.18$ & $<0.14$ & $<0.16$ & $<0.21$ \\
Event 4 90\% Processed & $<0.18$ & $<0.14$ & $<0.16$ & $<0.21$ \\
\hline \hline \multicolumn{5}{|c|}{ MRL* }
\end{tabular}


Table S28. HANs detected after chloramine or chlorine treatment in samples collected during decentralized treatment of greywater.

\begin{tabular}{|c|c|c|c|c|}
\hline Sample & TCAN & DCAN & BCAN & DBAN \\
\hline \multicolumn{5}{|c|}{ Feed: Chloramines } \\
\hline Event 1 & $<0.18$ & $3.0 \pm 0.1$ & $0.21 \pm 0.01$ & $<0.21$ \\
\hline Event 2 & $<0.18$ & $3.7 \pm 0.3$ & $0.17 \pm 0.013$ & $<0.21$ \\
\hline Event 3 & $<0.18$ & $2.4 \pm 0.1$ & $<0.16$ & $<0.21$ \\
\hline Event $420 \%$ Processed & $<0.18$ & $4.6 \pm 0.1$ & $0.27 \pm 0.06$ & $<0.21$ \\
\hline Event $450 \%$ Processed & $<0.18$ & $5.7 \pm 0.2$ & $0.51 \pm 0.06$ & $<0.21$ \\
\hline Event $490 \%$ Processed & $<0.18$ & $7.4 \pm 0.3$ & $<0.16$ & $<0.21$ \\
\hline \multicolumn{5}{|c|}{ Between FO and RO: Chloramines } \\
\hline Event 1 & $<0.18$ & $0.39 \pm 0.04$ & $<0.16$ & $<0.21$ \\
\hline Event 2 & $<0.18$ & $1.1 \pm 0.1$ & $<0.16$ & $<0.21$ \\
\hline Event 3 & $<0.18$ & $1.2 \pm 0.1$ & $<0.16$ & $<0.21$ \\
\hline Event $420 \%$ Processed & $<0.18$ & $0.62 \pm 0.09$ & $<0.16$ & $<0.21$ \\
\hline Event $450 \%$ Processed & $<0.18$ & $0.43 \pm 0.03$ & $<0.16$ & $<0.21$ \\
\hline Event $490 \%$ Processed & $<0.18$ & $0.55 \pm 0.12$ & $<0.16$ & $<0.21$ \\
\hline \multicolumn{5}{|c|}{ RO effluent: Chloramines } \\
\hline Event 1 & $<0.18$ & $<0.14$ & $<0.16$ & $<0.21$ \\
\hline Event 2 & $<0.18$ & $0.62 \pm 0.03$ & $<0.16$ & $<0.21$ \\
\hline Event 3 & $<0.18$ & $0.19 \pm 0.02$ & $<0.16$ & $<0.21$ \\
\hline Event $420 \%$ Processed & $<0.18$ & $<0.14$ & $<0.16$ & $<0.21$ \\
\hline Event $450 \%$ Processed & $<0.18$ & $1.2 \pm 0.1$ & $<0.16$ & $<0.21$ \\
\hline Event $490 \%$ Processed & $<0.18$ & $<0.14$ & $<0.16$ & $<0.21$ \\
\hline \multicolumn{5}{|c|}{ RO effluent: Free Chlorine } \\
\hline Event 1 & $<0.18$ & $0.40 \pm 0.02$ & $<0.16$ & $<0.21$ \\
\hline Event 2 & $<0.18$ & $0.42 \pm 0.03$ & $<0.16$ & $<0.21$ \\
\hline Event 3 & $<0.18$ & $0.23 \pm 0.01$ & $<0.16$ & $<0.21$ \\
\hline Event $420 \%$ Processed & $<0.18$ & $0.14 \pm 0.01$ & $<0.16$ & $<0.21$ \\
\hline Event $450 \%$ Processed & $<0.18$ & $0.29 \pm 0.04$ & $<0.16$ & $<0.21$ \\
\hline Event $490 \%$ Processed & $<0.18$ & $0.32 \pm 0.01$ & $<0.16$ & $<0.21$ \\
\hline MRL* & 0.18 & 0.14 & 0.16 & 0.21 \\
\hline
\end{tabular}


Table S29. HANs detected in samples collected during decentralized treatment of sewage.

\begin{tabular}{|c|c|c|c|c|}
\hline Sample & TCAN & DCAN & BCAN & DBAN \\
\hline \multicolumn{5}{|c|}{ Feed } \\
\hline Event 1 & $<0.18$ & $0.15 \pm 0.02$ & $<0.16$ & $<0.21$ \\
\hline Event 2 & $<0.18$ & $0.14 \pm 0.01$ & $<0.16$ & $<0.21$ \\
\hline Event 3 & $<0.18$ & $0.14 \pm 0.01$ & $<0.16$ & $<0.21$ \\
\hline Event $420 \%$ Processed & $<0.18$ & $0.22 \pm 0.03$ & $<0.16$ & $<0.21$ \\
\hline Event $450 \%$ Processed & $<0.18$ & $0.14 \pm 0.01$ & $<0.16$ & $<0.21$ \\
\hline Event $490 \%$ Proc & $<0.18$ & $0.14 \pm 0.01$ & $<0.16$ & $<0.21$ \\
\hline \multicolumn{5}{|c|}{ Between FO and RO } \\
\hline Event 1 & $<0.18$ & $0.14 \pm 0.01$ & $<0.16$ & $<0.21$ \\
\hline Event 2 & $<0.18$ & $0.14 \pm 0.01$ & $<0.16$ & $<0.21$ \\
\hline Event 3 & $<0.18$ & $0.14 \pm 0.01$ & $<0.16$ & $<0.21$ \\
\hline Event $420 \%$ Processed & $<0.18$ & $0.16 \pm 0.01$ & $<0.16$ & $<0.21$ \\
\hline Event $450 \%$ Processed & $<0.18$ & $0.14 \pm 0.01$ & $<0.16$ & $<0.21$ \\
\hline Event $490 \%$ Processed & $<0.18$ & $0.14 \pm 0.01$ & $<0.16$ & $<0.21$ \\
\hline \multicolumn{5}{|c|}{ RO effluent } \\
\hline Event 1 & $<0.18$ & $0.15 \pm 0.02$ & $<0.16$ & $<0.21$ \\
\hline Event 2 & $<0.18$ & $0.14 \pm 0.01$ & $<0.16$ & $<0.21$ \\
\hline Event 3 & $<0.18$ & $0.14 \pm 0.01$ & $<0.16$ & $<0.21$ \\
\hline Event $420 \%$ Processed & $<0.18$ & $0.22 \pm 0.02$ & $<0.16$ & $<0.21$ \\
\hline Event $450 \%$ Processed & $<0.18$ & $0.14 \pm 0.01$ & $<0.16$ & $<0.21$ \\
\hline Event $490 \%$ Processed & $<0.18$ & $0.14 \pm 0.01$ & $<0.16$ & $<0.21$ \\
\hline MRL* & 0.18 & 0.14 & 0.16 & 0.21 \\
\hline
\end{tabular}


Table S30. HANs detected after chloramine or chlorine treatment in samples collected during decentralized treatment of sewage.

\begin{tabular}{|c|c|c|c|c|}
\hline Sample & TCAN & DCAN & BCAN & $\mathrm{DBA}$ \\
\hline \multicolumn{5}{|c|}{ Feed: Chloramines } \\
\hline Event 1 & $<0.18$ & $0.93 \pm 0.14$ & $<0.16$ & $<0.21$ \\
\hline Event 2 & $<0.18$ & $0.90 \pm 0.03$ & $<0.16$ & $<0.21$ \\
\hline Event 3 & $<0.18$ & $0.78 \pm 0.16$ & $<0.16$ & $<0.21$ \\
\hline Event $420 \%$ Processed & $<0.18$ & $0.92 \pm 0.21$ & $<0.16$ & $<0.21$ \\
\hline $450 \%$ Processed & $<0.18$ & $0.45 \pm 0.03$ & $<0.16$ & $<0.21$ \\
\hline t $490 \%$ Processed & $<0.18$ & $1.6 \pm 0.1$ & $<0.16$ & $<0.21$ \\
\hline \multicolumn{5}{|c|}{ Between FO and RO: Chloramines } \\
\hline Eve & $<0.18$ & $0.16 \pm 0.03$ & $<0.16$ & 0.21 \\
\hline 2 & $<0.18$ & $0.66 \pm$ & $<0.16$ & $<0.21$ \\
\hline 3 & $<0.18$ & $0.18 \pm$ & $<0.16$ & $<0.21$ \\
\hline 420 & $<0$. & $0.32 \pm$ & $<0.16$ & $<0.21$ \\
\hline $\mathrm{Ev}$ & $<0.18$ & $0.60 \pm$ & $<0.16$ & $<0.21$ \\
\hline $490 \%$ & $<0.18$ & $1.3 \pm 0.3$ & $<0.16$ & $<0.21$ \\
\hline \multicolumn{5}{|c|}{ RO effluent: Chloramines } \\
\hline 1 & $<0.18$ & $0.16 \pm 0.03$ & $<0.16$ & $<0.21$ \\
\hline 2 & $<0.18$ & $0.14 \pm$ & $<0.16$ & $<0.21$ \\
\hline Event 3 & $<0.18$ & $0.16 \pm$ & $<0.16$ & $<0.21$ \\
\hline Event $420 \%$ Processed & $<0.18$ & $0.16 \pm 0.03$ & $<0.16$ & $<0.21$ \\
\hline $450 \%$ Processed & $<0.18$ & $0.15 \pm 0.01$ & $<0.16$ & $<0.21$ \\
\hline $490 \%$ Processed & $<0.18$ & $0.16 \pm 0.02$ & $<0.16$ & $<0.21$ \\
\hline \multicolumn{5}{|c|}{ RO effluent: Free Chlorine } \\
\hline 1 & $<0.18$ & $0.40 \pm 0.03$ & $<0.16$ & $<0.21$ \\
\hline Event 2 & $<0.18$ & $0.28 \pm 0.11$ & $<0.16$ & $<0.21$ \\
\hline Eve & $<0.18$ & $0.52 \pm 0.08$ & $<0.16$ & $<0.21$ \\
\hline $420 \%$ Pro & $<0.18$ & $0.52 \pm 0.02$ & $<0.16$ & $<0.21$ \\
\hline Event $450 \%$ Processed & $<0.18$ & $0.67 \pm 0.14$ & $<0.16$ & $<0.21$ \\
\hline Event $490 \%$ Processed & $<0.18$ & $1.2 \pm 0.2$ & $<0.16$ & $<0.21$ \\
\hline MRL* & 0.18 & 0.14 & 0.16 & 0.21 \\
\hline
\end{tabular}


Table S31. HKs and HNMs detected in samples collected during decentralized treatment of greywater.

\begin{tabular}{|c|c|c|c|}
\hline Sample & 1,1,1-TCP & 1,1-DCP & TCNM \\
\hline \multicolumn{4}{|c|}{ Feed } \\
\hline Event 1 & $0.33 \pm 0.06$ & $<0.22$ & $<0.16$ \\
\hline Event 2 & $<0.18$ & $0.22 \pm 0.01$ & $<0.16$ \\
\hline Event 3 & $<0.18$ & $<0.22$ & $<0.16$ \\
\hline Event $420 \%$ Processed & $<0.18$ & $0.22 \pm 0.02$ & $<0.16$ \\
\hline Event $450 \%$ Processed & $<0.18$ & $0.22 \pm 0.01$ & $<0.16$ \\
\hline Event $490 \%$ Processed & $<0.18$ & $0.46 \pm 0.03$ & $<0.16$ \\
\hline \multicolumn{4}{|c|}{ Between FO and RO } \\
\hline Event 1 & $<0.18$ & $<0.22$ & $<0.16$ \\
\hline Event 2 & $<0.18$ & $<0.22$ & $<0.16$ \\
\hline Event 3 & $<0.18$ & $<0.22$ & $<0.16$ \\
\hline Event $420 \%$ Processed & $<0.18$ & $<0.22$ & $<0.16$ \\
\hline Event $450 \%$ Processed & $<0.18$ & $<0.22$ & $<0.16$ \\
\hline Event $490 \%$ Processed & $<0.18$ & $<0.22$ & $<0.16$ \\
\hline \multicolumn{4}{|c|}{ RO effluent } \\
\hline Event 1 & $<0.18$ & $<0.22$ & $<0.16$ \\
\hline Event 2 & $<0.18$ & $<0.22$ & $<0.16$ \\
\hline Event 3 & $0.35 \pm 0.04$ & $<0.22$ & $<0.16$ \\
\hline Event $420 \%$ Processed & $<0.18$ & $<0.22$ & $<0.16$ \\
\hline Event $450 \%$ Processed & $0.26 \pm 0.01$ & $<0.22$ & $<0.16$ \\
\hline Event $490 \%$ Processed & $0.18 \pm 0.01$ & $<0.22$ & $<0.16$ \\
\hline MRL* & 0.18 & 0.22 & 0.16 \\
\hline
\end{tabular}


Table S32. HKs and HNMs detected after chloramine or chlorine treatment in samples collected during decentralized treatment of greywater.

\begin{tabular}{|c|c|c|c|}
\hline Sample & $1,1,1-\mathrm{TCP}$ & 1,1-DCP & TCNM \\
\hline \multicolumn{4}{|c|}{ Feed: Chloramines } \\
\hline Event 1 & $2.8 \pm 0.1$ & $8.7 \pm 0.3$ & $1.3 \pm 0.1$ \\
\hline Event 2 & $3.3 \pm 0.3$ & $14.7 \pm 1.2$ & $0.49 \pm 0.08$ \\
\hline Event 3 & $1.8 \pm 0.2$ & $10.6 \pm 0.5$ & $<0.16$ \\
\hline Event $420 \%$ Processed & $7.9 \pm 0.2$ & $16.5 \pm 1.7$ & $0.40 \pm 0.02$ \\
\hline Event $450 \%$ Processed & $10.8 \pm 0.5$ & $19.8 \pm 0.2$ & $1.1 \pm 0.2$ \\
\hline Event $490 \%$ Processed & $4.9 \pm 0.7$ & $25.9 \pm 2.0$ & $<0.16$ \\
\hline \multicolumn{4}{|c|}{ Between FO and RO: Chloramines } \\
\hline Event 1 & $<0.18$ & $1.7 \pm 0.2$ & $<0.16$ \\
\hline Event 2 & $<0.18$ & $4.2 \pm 0.6$ & $<0.16$ \\
\hline Event 3 & $<0.18$ & $3.9 \pm 0.3$ & $<0.16$ \\
\hline Event $420 \%$ Processed & $<0.18$ & $1.9 \pm 0.1$ & $<0.16$ \\
\hline Event $450 \%$ Processed & $<0.18$ & $1.8 \pm 0.1$ & $<0.16$ \\
\hline Event $490 \%$ Processed & $<0.18$ & $2.0 \pm 0.2$ & $<0.16$ \\
\hline \multicolumn{4}{|c|}{ RO effluent: Chloramines } \\
\hline Event 1 & $<0.18$ & $1.1 \pm 0.1$ & $<0.16$ \\
\hline Event 2 & $<0.18$ & $0.59 \pm 0.10$ & $<0.16$ \\
\hline Event 3 & $<0.18$ & $0.85 \pm 0.15$ & $<0.16$ \\
\hline Event $420 \%$ Processed & $<0.18$ & $0.82 \pm 0.04$ & $<0.16$ \\
\hline Event $450 \%$ Processed & $<0.18$ & $1.4 \pm 0.6$ & $0.65 \pm 0.06$ \\
\hline Event $490 \%$ Processed & $0.26 \pm 0.02$ & $0.94 \pm 0.04$ & $<0.16$ \\
\hline \multicolumn{4}{|c|}{ RO effluent: Free Chlorine } \\
\hline Event 1 & $0.79 \pm 0.10$ & $0.22 \pm 0.01$ & $0.40 \pm 0.04$ \\
\hline Event 2 & $0.67 \pm 0.08$ & $0.22 \pm 0.01$ & $<0.16$ \\
\hline Event 3 & $7.6 \pm 1.0$ & $0.28 \pm 0.04$ & $0.54 \pm 0.04$ \\
\hline Event $420 \%$ Processed & $<0.18$ & $<0.22$ & $0.27 \pm 0.03$ \\
\hline Event $450 \%$ Processed & $1.2 \pm 0.1$ & $<0.22$ & $0.41 \pm 0.03$ \\
\hline Event $490 \%$ Processed & $3.1 \pm 0.3$ & $0.22 \pm 0.01$ & $1.3 \pm 0.1$ \\
\hline MRL* & 0.18 & 0.22 & 0.16 \\
\hline
\end{tabular}


Table S33. HKs and HNMs detected in samples collected during decentralized treatment of sewage.

\begin{tabular}{|c|c|c|c|}
\hline Sample & $1,1,1-\mathrm{TCP}$ & 1,1-DCP & TCNM \\
\hline \multicolumn{4}{|c|}{ Feed } \\
\hline Event 1 & $<0.18$ & $<0.22$ & $<0.16$ \\
\hline Event 2 & $<0.18$ & $<0.22$ & $<0.16$ \\
\hline Event 3 & $<0.18$ & $<0.22$ & $<0.16$ \\
\hline Event $420 \%$ Processed & $<0.18$ & $<0.22$ & $<0.16$ \\
\hline Event $450 \%$ Processed & $<0.18$ & $<0.22$ & $<0.16$ \\
\hline Event $490 \%$ Processed & $<0.18$ & $<0.22$ & $<0.16$ \\
\hline \multicolumn{4}{|c|}{ Between FO and RO } \\
\hline Event 1 & $<0.18$ & $<0.22$ & $<0.16$ \\
\hline Event 2 & $<0.18$ & $<0.22$ & $<0.16$ \\
\hline Event 3 & $<0.18$ & $<0.22$ & $<0.16$ \\
\hline Event $420 \%$ Processed & $<0.18$ & $<0.22$ & $<0.16$ \\
\hline Event $450 \%$ Processed & $<0.18$ & $<0.22$ & $<0.16$ \\
\hline Event $490 \%$ Processed & $<0.18$ & $<0.22$ & $<0.16$ \\
\hline \multicolumn{4}{|c|}{ RO effluent } \\
\hline Event 1 & $<0.18$ & $<0.22$ & $<0.16$ \\
\hline Event 2 & $<0.18$ & $<0.22$ & $<0.16$ \\
\hline Event 3 & $<0.18$ & $<0.22$ & $<0.16$ \\
\hline Event $420 \%$ Processed & $<0.18$ & $<0.22$ & $<0.16$ \\
\hline Event $450 \%$ Processed & $<0.18$ & $<0.22$ & $<0.16$ \\
\hline Event $490 \%$ Processed & $<0.18$ & $<0.22$ & $<0.16$ \\
\hline MRL* & 0.18 & 0.22 & 0.16 \\
\hline
\end{tabular}


Table S34. HKs and HNMs detected after chloramine or chlorine treatment in samples collected during decentralized treatment of sewage.

\begin{tabular}{|c|c|c|c|}
\hline Sample & 1,1,1-TCP & 1,1-DCP & TCNM \\
\hline \multicolumn{4}{|c|}{ Feed: Chloramines } \\
\hline Event 1 & $<0.18$ & $6.2 \pm 0.3$ & $<0.16$ \\
\hline Event 2 & $<0.18$ & $5.9 \pm 0.7$ & $<0.16$ \\
\hline Event 3 & $<0.18$ & $5.0 \pm 0.3$ & $<0.16$ \\
\hline Event $420 \%$ Processed & $<0.18$ & $6.4 \pm 1.2$ & $<0.16$ \\
\hline Event $450 \%$ Processed & $<0.18$ & $2.7 \pm 0.1$ & $<0.16$ \\
\hline Event $490 \%$ Processed & $<0.18$ & $10.6 \pm 0.6$ & $<0.16$ \\
\hline \multicolumn{4}{|c|}{ Between FO and RO: Chloramines } \\
\hline Event 1 & $<0.18$ & $0.51 \pm 0.03$ & $<0.16$ \\
\hline Event 2 & $<0.18$ & $2.1 \pm 0.3$ & $<0.16$ \\
\hline Event 3 & $<0.18$ & $0.27 \pm 0.6$ & $<0.16$ \\
\hline Event $420 \%$ Processed & $<0.18$ & $0.60 \pm 0.12$ & $<0.16$ \\
\hline Event $450 \%$ Processed & $<0.18$ & $1.1 \pm 0.2$ & $<0.16$ \\
\hline Event $490 \%$ Processed & $<0.18$ & $1.2 \pm 0.2$ & $<0.16$ \\
\hline \multicolumn{4}{|c|}{ RO effluent: Chloramines } \\
\hline Event 1 & $<0.18$ & $0.31 \pm 0.13$ & $<0.16$ \\
\hline Event 2 & $<0.18$ & $0.42 \pm 0.01$ & $<0.16$ \\
\hline Event 3 & $<0.18$ & $0.27 \pm 0.04$ & $<0.16$ \\
\hline Event $420 \%$ Processed & $<0.18$ & $0.51 \pm 0.17$ & $<0.16$ \\
\hline Event $450 \%$ Processed & $<0.18$ & $0.47 \pm 0.15$ & $<0.16$ \\
\hline Event $490 \%$ Processed & $<0.18$ & $1.6 \pm 0.2$ & $<0.16$ \\
\hline \multicolumn{4}{|c|}{ RO effluent: Free Chlorine } \\
\hline Event 1 & $0.47 \pm 0.14$ & $5.2 \pm 0.6$ & $<0.16$ \\
\hline Event 2 & $1.2 \pm 0.1$ & $0.23 \pm 0.08$ & $<0.16$ \\
\hline Event 3 & $1.3 \pm 0.4$ & $0.61 \pm 0.15$ & $<0.16$ \\
\hline Event $420 \%$ Processed & $0.35 \pm 0.07$ & $<0.22$ & $0.23 \pm 0.04$ \\
\hline Event $450 \%$ Processed & $0.72 \pm 0.07$ & $<0.22$ & $0.82 \pm 0.05$ \\
\hline Event $490 \%$ Processed & $0.73 \pm 0.09$ & $<0.22$ & $1.2 \pm 0.1$ \\
\hline MRL* & 0.18 & 0.22 & 0.16 \\
\hline
\end{tabular}


Table S35. Lab scale FO membrane experiment descriptions. $N$-nitrosamines detected in samples collected during decentralized treatment of greywater.

\begin{tabular}{|c|c|c|c|c|c|c|c|c|}
\hline & NDMA & NDEA & NMOR & NPIP & NDBA & NDPA & NPYR & NMEA \\
\hline \hline \multicolumn{7}{|c|}{ Feed } \\
\hline Sample & $12.4 \pm 0.8$ & $<3.0$ & $13.8 \pm 2.5$ & $<2.3$ & $<2.3$ & $<3.0$ & $<3.0$ & $<2.1$ \\
Event 1 & $12.2 \pm 0.5$ & $<3.0$ & $26.4 \pm 2.8$ & $<2.3$ & $<2.3$ & $<3.0$ & $<3.0$ & $<2.1$ \\
Event 3 & $9.7 \pm 0.3$ & $<3.0$ & $25.4 \pm 2.9$ & $<2.3$ & $<2.3$ & $<3.0$ & $<3.0$ & $<2.1$ \\
Event 4 20\% Processed & $19.2 \pm 3.3$ & $<3.0$ & $34.6 \pm 2.2$ & $<2.3$ & $<2.3$ & $<3.0$ & $<3.0$ & $<2.1$ \\
Event 4 50\% Processed & $18.9 \pm 3.0$ & $<3.0$ & $36.6 \pm 1.5$ & $<2.3$ & $<2.3$ & $<3.0$ & $<3.0$ & $<2.1$ \\
Event 4 90\% Processed & $34.0 \pm 1.1$ & $<3.0$ & $53.4 \pm 1.6$ & $<2.3$ & $<2.3$ & $<3.0$ & $<3.0$ & $<2.1$ \\
\hline \multicolumn{7}{|c|}{ Between FO and RO } \\
\hline Event 1 & $30.4 \pm 9.8$ & $<3.0$ & $38.0 \pm 2.1$ & $<2.3$ & $<2.3$ & $<3.0$ & $<3.0$ & $<2.1$ \\
Event 2 & $68.0 \pm 6.3$ & $<3.0$ & $54.7 \pm 5.4$ & $<2.3$ & $<2.3$ & $<3.0$ & $<3.0$ & $<2.1$ \\
Event 3 & $44.2 \pm 4.5$ & $<3.0$ & $40.9 \pm 4.4$ & $<2.3$ & $<2.3$ & $<3.0$ & $<3.0$ & $<2.1$ \\
Event 4 20\% Processed & $169.7 \pm 2.5$ & $<3.0$ & $86.4 \pm 6.0$ & $<2.3$ & $<2.3$ & $<3.0$ & $<3.0$ & $<2.1$ \\
Event 4 50\% Processed & $194.0 \pm 7.0$ & $<3.0$ & $66.4 \pm 9.8$ & $<2.3$ & $<2.3$ & $<3.0$ & $<3.0$ & $<2.1$ \\
Event 4 90\% Processed & $254.5 \pm 27.9$ & $<3.0$ & $105.4 \pm 7.5$ & $<2.3$ & $<2.3$ & $<3.0$ & $<3.0$ & $<2.1$ \\
\hline \multicolumn{7}{|c|}{ RO effluent } \\
\hline Event 1 & $3.0 \pm 0.4$ & $<3.0$ & $<3.0$ & $<2.3$ & $<2.3$ & $<3.0$ & $<3.0$ & $<2.1$ \\
Event 2 & $3.1 \pm 0.3$ & $<3.0$ & $<3.0$ & $<2.3$ & $<2.3$ & $<3.0$ & $<3.0$ & $<2.1$ \\
Event 3 & $3.2 \pm 0.8$ & $<3.0$ & $<3.0$ & $<2.3$ & $<2.3$ & $<3.0$ & $<3.0$ & $<2.1$ \\
Event 4 20\% Processed & $3.6 \pm 0.3$ & $<3.0$ & $<3.0$ & $<2.3$ & $<2.3$ & $<3.0$ & $<3.0$ & $<2.1$ \\
Event 4 50\% Processed & $3.5 \pm 0.8$ & $<3.0$ & $<3.0$ & $<2.3$ & $<2.3$ & $<3.0$ & $<3.0$ & $<2.1$ \\
Event 4 90\% Processed & $3.4 \pm 0.1$ & $<3.0$ & $<3.0$ & $<2.3$ & $<2.3$ & $<3.0$ & $<3.0$ & $<2.1$ \\
\hline \hline
\end{tabular}


Table S36. $N$-nitrosamines detected after chloramine or chlorine treatment in samples collected during decentralized treatment of greywater.

\begin{tabular}{|c|c|c|c|c|c|c|c|c|}
\hline Sample & NDMA & NDEA & NMOR & NPIP & NDBA & NDPA & NPYR & NMEA \\
\hline \multicolumn{9}{|c|}{ Feed: Chloramines } \\
\hline Event 1 & $595.5 \pm 18.5$ & $<3.0$ & $17.3 \pm 4.1$ & $<2.3$ & $<2.3$ & $<3.0$ & $<3.0$ & $<2.1$ \\
\hline Event 2 & $3297.2 \pm 19.1$ & $<3.0$ & $23.6 \pm 4.0$ & $<2.3$ & $<2.3$ & $<3.0$ & $<3.0$ & $<2.1$ \\
\hline Event 3 & $4080.4 \pm 255.5$ & $<3.0$ & $56.7 \pm 1.2$ & $<2.3$ & $<2.3$ & $<3.0$ & $<3.0$ & $<2.1$ \\
\hline Event $420 \%$ Processed & $513.9 \pm 44.9$ & $<3.0$ & $28.2 \pm 3.5$ & $<2.3$ & $<2.3$ & $<3.0$ & $<3.0$ & $<2.1$ \\
\hline Event $450 \%$ Processed & $714.9 \pm 35.2$ & $<3.0$ & $28.0 \pm 5.6$ & $<2.3$ & $<2.3$ & $<3.0$ & $<3.0$ & $<2.1$ \\
\hline Event $490 \%$ Processed & $1581.6 \pm 107.7$ & $<3.0$ & $56.1 \pm 0.4$ & $<2.3$ & $<2.3$ & $<3.0$ & $<3.0$ & $<2.1$ \\
\hline \multicolumn{9}{|c|}{ Between FO and RO: Chloramines } \\
\hline Event 1 & $1214.8 \pm 150.2$ & $<3.0$ & $109.7 \pm 8.7$ & $<2.3$ & $<2.3$ & $<3.0$ & $<3.0$ & $<2.1$ \\
\hline Event 2 & $132.1 \pm 4.6$ & $<3.0$ & $50.5 \pm 4.2$ & $<2.3$ & $<2.3$ & $<3.0$ & $<3.0$ & $<2.1$ \\
\hline Event 3 & $454.7 \pm 11.1$ & $<3.0$ & $65.9 \pm 10.1$ & $<2.3$ & $<2.3$ & $<3.0$ & $<3.0$ & $<2.1$ \\
\hline Event $420 \%$ Processed & $1406.3 \pm 153.8$ & $<3.0$ & $115.4 \pm 13.3$ & $<2.3$ & $<2.3$ & $<3.0$ & $<3.0$ & $<2.1$ \\
\hline Event $450 \%$ Processed & $1165.4 \pm 4.8$ & $<3.0$ & $125.2 \pm 1.1$ & $<2.3$ & $<2.3$ & $<3.0$ & $<3.0$ & $<2.1$ \\
\hline Event $490 \%$ Processed & $1090.4 \pm 23.9$ & $<3.0$ & $123.3 \pm 33.4$ & $<2.3$ & $<2.3$ & $<3.0$ & $<3.0$ & $<2.1$ \\
\hline \multicolumn{9}{|c|}{ RO effluent: Chloramines } \\
\hline Event 1 & $4.9 \pm 0.7$ & $<3.0$ & $<3.0$ & $<2.3$ & $<2.3$ & $<3.0$ & $<3.0$ & $<2.1$ \\
\hline Event 2 & $5.7 \pm 1.6$ & $<3.0$ & $<3.0$ & $<2.3$ & $<2.3$ & $<3.0$ & $<3.0$ & $<2.1$ \\
\hline Event 3 & $91.0 \pm 8.7$ & $<3.0$ & $<3.0$ & $<2.3$ & $<2.3$ & $<3.0$ & $<3.0$ & $<2.1$ \\
\hline Event $420 \%$ Processed & $6.1 \pm 1.2$ & $<3.0$ & $<3.0$ & $<2.3$ & $<2.3$ & $<3.0$ & $<3.0$ & $<2.1$ \\
\hline Event $450 \%$ Processed & $6.0 \pm 0.1$ & $<3.0$ & $16.2 \pm 1.7$ & $<2.3$ & $<2.3$ & $<3.0$ & $<3.0$ & $<2.1$ \\
\hline Event $490 \%$ Processed & $147.8 \pm 11.8$ & $<3.0$ & $15.1 \pm 1.1$ & $<2.3$ & $<2.3$ & $<3.0$ & $<3.0$ & $<2.1$ \\
\hline \multicolumn{9}{|c|}{ RO effluent: Free Chlorine } \\
\hline Event 1 & $3.4 \pm 0.3$ & $<3.0$ & $10.0 \pm 1.8$ & $<2.3$ & $<2.3$ & $<3.0$ & $<3.0$ & $<2.1$ \\
\hline Event 2 & $7.3 \pm 0.3$ & $<3.0$ & $<3.0$ & $<2.3$ & $<2.3$ & $<3.0$ & $<3.0$ & $<2.1$ \\
\hline Event 3 & $27.0 \pm 0.3$ & $<3.0$ & $<3.0$ & $<2.3$ & $<2.3$ & $<3.0$ & $<3.0$ & $<2.1$ \\
\hline Event $420 \%$ Processed & $4.8 \pm 1.1$ & $<3.0$ & $<3.0$ & $<2.3$ & $<2.3$ & $<3.0$ & $<3.0$ & $<2.1$ \\
\hline Event $450 \%$ Processed & $3.2 \pm 0.4$ & $<3.0$ & $<3.0$ & $<2.3$ & $<2.3$ & $<3.0$ & $<3.0$ & $<2.1$ \\
\hline Event $490 \%$ Processed & $4.5 \pm 0.7$ & $<3.0$ & $<3.0$ & $<2.3$ & $<2.3$ & $<3.0$ & $<3.0$ & $<2.1$ \\
\hline MRL* & 2.0 & 3.0 & 3.0 & 2.3 & 2.3 & 3.0 & 3.0 & 2.1 \\
\hline
\end{tabular}


Table S37. $N$-nitrosamines detected in samples collected during decentralized treatment of sewage.

\begin{tabular}{|c|c|c|c|c|c|c|c|c|}
\hline & NDMA & NDEA & NMOR & NPIP & NDBA & NDPA & NPYR & NMEA \\
\hline \hline \multicolumn{7}{|c|}{ Feed } \\
\hline Sample & $14.9 \pm 2.7$ & $<3.0$ & $3.9 \pm 0.2$ & $<2.3$ & $<2.3$ & $<3.0$ & $217.0 \pm 4.2$ & $<2.1$ \\
Event 1 & $24.0 \pm 2.4$ & $<3.0$ & $3.8 \pm 0.1$ & $<2.3$ & $<2.3$ & $<3.0$ & $171.1 \pm 5.3$ & $<2.1$ \\
Event 3 & $9.6 \pm 2.3$ & $<3.0$ & $3.0 \pm 0.3$ & $<2.3$ & $<2.3$ & $<3.0$ & $118.1 \pm 16.3$ & $<2.1$ \\
Event 4 20\% Processed & $9.3 \pm 0.3$ & $<3.0$ & $4.0 \pm 0.2$ & $<2.3$ & $<2.3$ & $<3.0$ & $84.5 \pm 7.7$ & $<2.1$ \\
Event 4 50\% Processed & $11.4 \pm 2.0$ & $<3.0$ & $10.0 \pm 1.1$ & $<2.3$ & $<2.3$ & $<3.0$ & $140.5 \pm 17.1$ & $<2.1$ \\
Event 4 90\% Processed & $12.3 \pm 1.0$ & $<3.0$ & $17.4 \pm 2.8$ & $<2.3$ & $<2.3$ & $<3.0$ & $64.3 \pm 5.7$ & $<2.1$ \\
\hline \multicolumn{7}{|c|}{ Between FO and RO } \\
\hline Event 1 & $7.7 \pm 0.1$ & $<3.0$ & $3.0 \pm 0.2$ & $<2.3$ & $<2.3$ & $<3.0$ & $6.5 \pm 2.0$ & $<2.1$ \\
Event 2 & $18.6 \pm 1.3$ & $<3.0$ & $3.9 \pm 0.9$ & $<2.3$ & $<2.3$ & $<3.0$ & $13.5 \pm 0.1$ & $<2.1$ \\
Event 3 & $13.1 \pm 0.5$ & $<3.0$ & $3.2 \pm 0.2$ & $<2.3$ & $<2.3$ & $<3.0$ & $24.4 \pm 3.4$ & $<2.1$ \\
Event 4 20\% Processed & $128.0 \pm 5.8$ & $<3.0$ & $4.1 \pm 0.5$ & $<2.3$ & $<2.3$ & $<3.0$ & $1113.9 \pm 12.6$ & $<2.1$ \\
Event 4 50\% Processed & $156.8 \pm 9.1$ & $<3.0$ & $8.9 \pm 2.3$ & $<2.3$ & $<2.3$ & $<3.0$ & $1316.8 \pm 174.1$ & $<2.1$ \\
Event 4 90\% Processed & $147.7 \pm 23.4$ & $<3.0$ & $8.2 \pm 0.3$ & $<2.3$ & $<2.3$ & $<3.0$ & $1153.5 \pm 74.0$ & $<2.1$ \\
\hline \multicolumn{7}{|c|}{ RO effluent } \\
\hline Event 1 & $<2.0$ & $<3.0$ & $<3.0$ & $<2.3$ & $<2.3$ & $<3.0$ & $<3.0$ & $<2.1$ \\
Event 2 & $<2.0$ & $<3.0$ & $<3.0$ & $<2.3$ & $<2.3$ & $<3.0$ & $<3.0$ & $<2.1$ \\
Event 3 & $<2.0$ & $<3.0$ & $<3.0$ & $<2.3$ & $<2.3$ & $<3.0$ & $<3.0$ & $<2.1$ \\
Event 4 20\% Processed & $5.8 \pm 0.5$ & $<3.0$ & $<3.0$ & $<2.3$ & $<2.3$ & $<3.0$ & $<3.0$ & $<2.1$ \\
Event 4 50\% Processed & $<2.0$ & $<3.0$ & $<3.0$ & $<2.3$ & $<2.3$ & $<3.0$ & $<3.0$ & $<2.1$ \\
Event 4 90\% Processed & $5.1 \pm 0.3$ & $<3.0$ & $<3.0$ & $<2.3$ & $<2.3$ & $<3.0$ & $<3.0$ & $<2.1$ \\
\hline \hline
\end{tabular}


Table S38. $N$-nitrosamines detected after chloramine or chlorine treatment in samples collected during decentralized treatment of sewage.

\begin{tabular}{|c|c|c|c|c|c|c|c|c|}
\hline Sample & NDMA & NDEA & NMOR & NPIP & NDBA & NDPA & NPYR & NMEA \\
\hline \multicolumn{9}{|c|}{ Feed: Chloramines } \\
\hline Event 1 & $30.6 \pm 4.1$ & $<3.0$ & $3.0 \pm 0.1$ & $<2.3$ & $<2.3$ & $<3.0$ & $196.3 \pm 16.0$ & $<2.1$ \\
\hline Event 2 & $15.4 \pm 2.2$ & $<3.0$ & $<3.0$ & $<2.3$ & $<2.3$ & $<3.0$ & $128.8 \pm 6.1$ & $<2.1$ \\
\hline Event 3 & $21.9 \pm 0.5$ & $<3.0$ & $<3.0$ & $<2.3$ & $<2.3$ & $<3.0$ & $262.7 \pm 5.9$ & $<2.1$ \\
\hline Event $420 \%$ Processed & $8.9 \pm 0.3$ & $<3.0$ & $5.2 \pm 0.2$ & $<2.3$ & $<2.3$ & $<3.0$ & $98.0 \pm 4.6$ & $<2.1$ \\
\hline Event $450 \%$ Processed & $15.1 \pm 1.4$ & $<3.0$ & $8.7 \pm 0.2$ & $<2.3$ & $<2.3$ & $<3.0$ & $187.1 \pm 15.0$ & $<2.1$ \\
\hline Event $490 \%$ Processed & $25.9 \pm 0.7$ & $<3.0$ & $21.2 \pm 0.4$ & $<2.3$ & $<2.3$ & $<3.0$ & $221.2 \pm 6.9$ & $<2.1$ \\
\hline \multicolumn{9}{|c|}{ Between FO and RO: Chloramines } \\
\hline Event 1 & $12.4 \pm 2.9$ & $<3.0$ & $4.4 \pm 0.2$ & $<2.3$ & $<2.3$ & $<3.0$ & $6.2 \pm 0.8$ & $<2.1$ \\
\hline Event 2 & $19.3 \pm 0.6$ & $<3.0$ & $3.0 \pm 0.1$ & $<2.3$ & $<2.3$ & $<3.0$ & $<3.0$ & $<2.1$ \\
\hline Event 3 & $17.4 \pm 2.0$ & $<3.0$ & $4.1 \pm 0.3$ & $<2.3$ & $<2.3$ & $<3.0$ & $25.1 \pm 0.8$ & $<2.1$ \\
\hline Event $420 \%$ Processed & $215.8 \pm 27.6$ & $<3.0$ & $3.0 \pm 0.1$ & $<2.3$ & $<2.3$ & $<3.0$ & $1221.5 \pm 27.8$ & $<2.1$ \\
\hline Event $450 \%$ Processed & $217.8 \pm 24.5$ & $<3.0$ & $3.0 \pm 0.1$ & $<2.3$ & $<2.3$ & $<3.0$ & $1084.2 \pm 50.8$ & $<2.1$ \\
\hline Event $490 \%$ Processed & $435.3 \pm 16.5$ & $<3.0$ & $4.1 \pm 1.1$ & $<2.3$ & $<2.3$ & $<3.0$ & $2041.7 \pm 114.3$ & $<2.1$ \\
\hline \multicolumn{9}{|c|}{ RO effluent: Chloramines } \\
\hline Event 1 & $2.3 \pm 0.4$ & $<3.0$ & $<3.0$ & $<2.3$ & $<2.3$ & $<3.0$ & $<3.0$ & $<2.1$ \\
\hline Event 2 & $5.0 \pm 0.2$ & $<3.0$ & $<3.0$ & $<2.3$ & $<2.3$ & $<3.0$ & $<3.0$ & $<2.1$ \\
\hline Event 3 & $2.7 \pm 0.4$ & $<3.0$ & $<3.0$ & $<2.3$ & $<2.3$ & $<3.0$ & $<3.0$ & $<2.1$ \\
\hline Event $420 \%$ Processed & $47.2 \pm 2.0$ & $<3.0$ & $<3.0$ & $<2.3$ & $<2.3$ & $<3.0$ & $8.4 \pm 0.3$ & $<2.1$ \\
\hline Event $450 \%$ Processed & $17.6 \pm 2.7$ & $<3.0$ & $<3.0$ & $<2.3$ & $<2.3$ & $<3.0$ & $<3.0$ & $<2.1$ \\
\hline Event $490 \%$ Processed & $19.8 \pm 2.0$ & $<3.0$ & $<3.0$ & $<2.3$ & $<2.3$ & $<3.0$ & $<3.0$ & $<2.1$ \\
\hline \multicolumn{9}{|c|}{ RO effluent: Free Chlorine } \\
\hline Event 1 & $<2.0$ & $<3.0$ & $<3.0$ & $<2.3$ & $<2.3$ & $<3.0$ & $<3.0$ & $<2.1$ \\
\hline Event 2 & $4.4 \pm 0.5$ & $<3.0$ & $<3.0$ & $<2.3$ & $<2.3$ & $<3.0$ & $<3.0$ & $<2.1$ \\
\hline Event 3 & $30.0 \pm 1.6$ & $<3.0$ & $<3.0$ & $<2.3$ & $<2.3$ & $<3.0$ & $<3.0$ & $<2.1$ \\
\hline Event $420 \%$ Processed & $11.4 \pm 0.5$ & $<3.0$ & $<3.0$ & $<2.3$ & $<2.3$ & $<3.0$ & $3.9 \pm 0.8$ & $<2.1$ \\
\hline Event $450 \%$ Processed & $6.5 \pm 1.5$ & $<3.0$ & $<3.0$ & $<2.3$ & $<2.3$ & $<3.0$ & $3.0 \pm 0.7$ & $<2.1$ \\
\hline Event $490 \%$ Processed & $13.7 \pm 1.1$ & $<3.0$ & $<3.0$ & $<2.3$ & $<2.3$ & $<3.0$ & $3.0 \pm 0.7$ & $<2.1$ \\
\hline MRL* & 2.0 & 3.0 & 3.0 & 2.3 & 2.3 & 3.0 & 3.0 & 2.1 \\
\hline
\end{tabular}


Tables S39-S54. DBPs detected during centralized treatment of greywater and sewage. Error bars represent ranges for duplicate measurements for halogenated DBPs, and $N$-nitrosamines, where applicable. $N . M .=$ not measured. 
Table S39. THM4 detected in samples collected during centralized treatment.

\begin{tabular}{|c|c|c|c|c|}
\hline Sample & TCM & BDCM & DBCM & TBM \\
\hline \hline \multicolumn{5}{|c|}{ FO/RO Feed (RO concentrate) } \\
\hline Event 1 & $38.9 \pm 2.0$ & $12.9 \pm 1.5$ & $3.0 \pm 0.3$ & $0.64 \pm 0.08$ \\
Event 2 & $35.4 \pm 1.4$ & $23.7 \pm 0.1$ & $6.6 \pm 0.1$ & $1.0 \pm 0.1$ \\
Event 3 & $46.9 \pm 4.5$ & $25.7 \pm 3.8$ & $7.2 \pm 1.4$ & $1.2 \pm 0.3$ \\
Event 4 & $27.0 \pm 1.1$ & $14.9 \pm 0.3$ & $4.1 \pm 0.1$ & $0.72 \pm 0.04$ \\
\hline \multicolumn{5}{|c|}{ FO/RO Permeate } \\
\hline Event 1 & $1.0 \pm 0.1$ & $0.64 \pm 0.01$ & $0.16 \pm 0.01$ & $<0.21$ \\
Event 2 & $0.81 \pm 0.21$ & $0.66 \pm 0.11$ & $0.23 \pm 0.06$ & $<0.21$ \\
Event 3 & $8.7 \pm 0.2$ & $4.6 \pm 0.1$ & $1.0 \pm 0.1$ & $<0.21$ \\
Event 4 & $5.8 \pm 0.3$ & $4.2 \pm 0.2$ & $1.2 \pm 0.1$ & $0.21 \pm 0.01$ \\
\hline \multicolumn{5}{|c|}{ RO Feed } \\
\hline Event 1 & $N . M$ & $N . M$. & $N . M$. & $N . M$ \\
Event 2 & 6.7 & 3.9 & 1.7 & 0.22 \\
Event 3 & 6.8 & 3.7 & 1.1 & 0.21 \\
Event 4 & 5.1 & 2.7 & 0.83 & $<0.21$ \\
\hline \multicolumn{5}{|c|}{ RO Permeate } \\
\hline Event 1 & 1.3 & 0.85 & 0.19 & $<0.21$ \\
Event 2 & 2.0 & 1.2 & 0.52 & $<0.21$ \\
Event 3 & 3.4 & 2.4 & 0.71 & $<0.21$ \\
Event 4 & 2.2 & 1.5 & 0.46 & $<0.21$ \\
\hline \hline MRL* & 0.24 & 0.18 & 0.16 & 0.21 \\
\hline
\end{tabular}


Table S40. THM4 detected after chloramine or chlorine treatment in samples collected during centralized treatment.

\begin{tabular}{|c|c|c|c|c|}
\hline Sample & TCM & BDCM & DBCM & TBM \\
\hline \hline \multicolumn{5}{|c|}{ Chloramines: FO/RO Feed (RO concentrate) } \\
\hline Event 1 & $41.3 \pm 4.1$ & $18.2 \pm 1.3$ & $5.6 \pm 0.3$ & $1.1 \pm 0.1$ \\
Event 2 & $22.9 \pm 0.2$ & $20.1 \pm 0.2$ & $7.1 \pm 0.2$ & $1.1 \pm 0.1$ \\
Event 3 & $56.0 \pm 2.5$ & $27.7 \pm 2.1$ & $9.1 \pm 1.1$ & $1.3 \pm 0.2$ \\
Event 4 & $48.7 \pm 0.1$ & $21.2 \pm 0.1$ & $6.3 \pm 0.1$ & $2.3 \pm 0.1$ \\
\hline \multicolumn{5}{|c|}{ Chloramines: FO/RO Permeate } \\
\hline Event 1 & $0.81 \pm 0.21$ & $0.67 \pm 0.11$ & $0.23 \pm 0.06$ & $<0.21$ \\
Event 2 & $1.4 \pm 0.1$ & $0.27 \pm 0.02$ & $0.16 \pm 0.01$ & $<0.21$ \\
Event 3 & $7.0 \pm 0.5$ & $3.9 \pm 0.1$ & $0.94 \pm 0.09$ & $0.21 \pm 0.01$ \\
Event 4 & $9.6 \pm 0.1$ & $3.8 \pm 0.1$ & $1.2 \pm 0.1$ & $1.4 \pm 0.1$ \\
\hline \multicolumn{5}{|c|}{ Chloramines: RO Feed } \\
\hline Event 1 & $N . M$. & $N . M$. & $N . M$. & $N . M$. \\
Event 2 & 6.7 & 7.3 & 3.3 & 0.91 \\
Event 3 & 10.7 & 7.4 & 2.9 & 0.74 \\
Event 4 & 14.8 & 7.1 & 3.0 & 2.2 \\
\hline \multicolumn{5}{|c|}{ Chloramines: RO Permeate } \\
\hline Event 1 & 1.2 & 0.93 & 0.23 & $<0.21$ \\
Event 2 & 1.6 & 1.0 & 0.47 & $<0.21$ \\
Event 3 & 3.1 & 2.4 & 0.67 & $<0.21$ \\
Event 4 & 7.5 & 1.6 & 0.47 & 1.3 \\
\hline \hline MRL* & 0.24 & 0.18 & 0.16 & 0.21 \\
\hline
\end{tabular}


Table S41. HAAs detected in samples collected during centralized treatment.

\begin{tabular}{|c|c|c|c|c|c|c|c|c|c|c|}
\hline Sample & CAA & BAA & DCAA & BCAA & DBAA & TCAA & BDCAA & CDBAA & TBAA & IAA \\
\hline \multicolumn{11}{|c|}{ FO/RO Feed (RO concentrate) } \\
\hline Event 1 & $9.3 \pm 1.6$ & $<0.20$ & $42.7 \pm 1.9$ & $13.4 \pm 0.4$ & $2.7 \pm 0.1$ & $71.5 \pm 0.1$ & $<0.22$ & $0.51 \pm 0.05$ & $<0.24$ & $2.9 \pm 0.2$ \\
\hline Event 2 & $2.3 \pm 0.6$ & $1.6 \pm 0.2$ & $31.7 \pm 2.5$ & $6.5 \pm 0.4$ & $1.7 \pm 0.5$ & $26.5 \pm 2.6$ & $0.64 \pm 0.32$ & $0.66 \pm 0.21$ & $<0.24$ & $<0.20$ \\
\hline Event 3 & $13.8 \pm 3.2$ & $1.4 \pm 0.2$ & $54.4 \pm 6.9$ & $21.2 \pm 2.7$ & $5.4 \pm 0.9$ & $46.1 \pm 6.5$ & $24.0 \pm 4.6$ & $14.1 \pm 1.0$ & $<0.24$ & $2.2 \pm 0.1$ \\
\hline Event 4 & $6.7 \pm 1.7$ & $1.0 \pm 0.3$ & $45.5 \pm 0.4$ & $11.4 \pm 1.0$ & $2.7 \pm 0.2$ & $46.2 \pm 2.1$ & $15.5 \pm 2.3$ & $11.6 \pm 2.1$ & $<0.24$ & $1.2 \pm 0.1$ \\
\hline \multicolumn{11}{|c|}{ FO/RO Permeate } \\
\hline Event 1 & $<0.22$ & $<0.20$ & $<0.17$ & $<0.16$ & $<0.18$ & $<0.18$ & $<0.22$ & $<0.22$ & $<0.24$ & $<0.20$ \\
\hline Event 2 & $<0.22$ & $<0.20$ & $0.81 \pm 0.20$ & $<0.16$ & $<0.18$ & $<0.18$ & $<0.22$ & $<0.22$ & $<0.24$ & $<0.20$ \\
\hline Event 3 & $<0.22$ & $<0.20$ & $<0.17$ & $<0.16$ & $<0.18$ & $<0.18$ & $<0.22$ & $<0.22$ & $<0.24$ & $<0.20$ \\
\hline Event 4 & $<0.22$ & $<0.20$ & $0.64 \pm 0.31$ & $<0.16$ & $<0.18$ & $<0.18$ & $<0.22$ & $<0.22$ & $<0.24$ & $<0.20$ \\
\hline \multicolumn{11}{|c|}{ RO Feed } \\
\hline Event 1 & N.M. & N.M. & N.M. & N.M. & N.M. & N.M. & N.M. & N.M. & $N . M$. & N.M. \\
\hline Event 2 & 2.8 & 1.4 & 53.6 & 10.1 & 1.9 & 47.3 & 0.29 & 0.22 & $<0.24$ & $<0.20$ \\
\hline Event 3 & 2.0 & 0.21 & 4.8 & 1.3 & 0.24 & 5.4 & $<0.22$ & $<0.22$ & $<0.24$ & $<0.20$ \\
\hline Event 4 & 1.6 & $<0.20$ & 7.1 & 0.45 & $<0.18$ & 5.7 & 5.2 & $<0.22$ & $<0.24$ & $<0.20$ \\
\hline \multicolumn{11}{|c|}{ RO Permeate } \\
\hline Event 1 & $<0.22$ & $<0.20$ & $<0.17$ & $<0.16$ & $<0.18$ & $<0.18$ & $<0.22$ & $<0.22$ & $<0.24$ & $<0.20$ \\
\hline Event 2 & 0.65 & 0.27 & 0.79 & 0.14 & 0.30 & 0.68 & $<0.22$ & $<0.22$ & $<0.24$ & $<0.20$ \\
\hline Event 3 & $<0.22$ & $<0.20$ & 1.3 & $<0.16$ & $<0.18$ & $<0.18$ & $<0.22$ & $<0.22$ & $<0.24$ & $<0.20$ \\
\hline Event 4 & $<0.22$ & $<0.20$ & $<0.17$ & $<0.16$ & $<0.18$ & $<0.18$ & $<0.22$ & $<0.22$ & $<0.24$ & $<0.20$ \\
\hline MRL* & 0.22 & 0.20 & 0.17 & 0.16 & 0.18 & 0.18 & 0.22 & 0.22 & 0.24 & 0.20 \\
\hline
\end{tabular}

Table S42. HAAs detected after chloramine treatment in samples collected during centralized treatment.

\begin{tabular}{|c|c|c|c|c|c|c|c|c|c|c|}
\hline Sample & CAA & BAA & DCAA & BCAA & DBAA & TCAA & BDCAA & CDBAA & TBAA & IAA \\
\hline \multicolumn{11}{|c|}{ Chloramines: FO/RO Feed (RO concentrate) } \\
\hline Event 1 & $21.8 \pm 1.7$ & $4.1 \pm 0.1$ & $123.0 \pm 2.0$ & $39.2 \pm 3.5$ & $10.4 \pm 1.9$ & $82.5 \pm 5.9$ & $18.5 \pm 1.7$ & $6.0 \pm 0.5$ & $0.51 \pm 0.06$ & $8.3 \pm 0.8$ \\
\hline Event 2 & $12.3 \pm 0.7$ & $3.0 \pm 0.1$ & $117.9 \pm 3.0$ & $25.9 \pm 0.7$ & $5.7 \pm 0.3$ & $49.4 \pm 0.1$ & $6.8 \pm 0.2$ & $2.6 \pm 0.1$ & $<0.24$ & $0.48 \pm 0.01$ \\
\hline Event 3 & $20.1 \pm 2.0$ & $3.0 \pm 0.1$ & $87.2 \pm 6.3$ & $31.2 \pm 2.7$ & $8.8 \pm 0.8$ & $45.3 \pm 0.2$ & $16.3 \pm 0.3$ & $14.0 \pm 5.0$ & $<0.24$ & $5.1 \pm 1.1$ \\
\hline Event 4 & $14.4 \pm 1.8$ & $2.1 \pm 0.2$ & $78.0 \pm 2.1$ & $30.4 \pm 0.7$ & $8.8 \pm 0.2$ & $42.9 \pm 0.6$ & $19.1 \pm 1.8$ & $21.4 \pm 3.6$ & $<0.24$ & $11.2 \pm 0.9$ \\
\hline \multicolumn{11}{|c|}{ Chloramines: FO/RO Permeate } \\
\hline Event 1 & $<0.22$ & $<0.20$ & $0.48 \pm 0.15$ & $<0.16$ & $<0.18$ & $<0.18$ & $<0.22$ & $<0.22$ & $<0.24$ & $<0.20$ \\
\hline Event 2 & $<0.22$ & $<0.20$ & $2.8 \pm 0.4$ & $<0.16$ & $<0.18$ & $<0.18$ & $<0.22$ & $<0.22$ & $<0.24$ & $<0.20$ \\
\hline Event 3 & $<0.22$ & $<0.20$ & $0.69 \pm 0.34$ & $<0.16$ & $<0.18$ & $<0.18$ & $<0.22$ & $<0.22$ & $<0.24$ & $<0.20$ \\
\hline Event 4 & $<0.22$ & $<0.20$ & $0.71 \pm 0.40$ & $<0.16$ & $<0.18$ & $<0.18$ & $<0.22$ & $<0.22$ & $<0.24$ & $<0.20$ \\
\hline \multicolumn{11}{|c|}{ Chloramines: RO Feed } \\
\hline Event 1 & N.M. & N.M. & N.M. & N.M. & N.M. & N.M. & N.M. & N.M. & N.M. & N.M. \\
\hline Event 2 & 1.1 & $<0.20$ & 19.2 & 7.4 & 3.2 & 7.6 & 0.54 & $<0.22$ & $<0.24$ & $<0.20$ \\
\hline Event 3 & 2.6 & $<0.20$ & 6.1 & 5.3 & 2.3 & 3.1 & $<0.22$ & $<0.22$ & $<0.24$ & 0.41 \\
\hline Event 4 & 4.0 & 1.2 & 16.3 & 9.0 & 3.6 & 6.9 & $<0.22$ & $<0.22$ & $<0.24$ & 0.58 \\
\hline \multicolumn{11}{|c|}{ Chloramines: RO Permeate } \\
\hline Event 1 & $<0.22$ & $<0.20$ & 0.98 & $<0.16$ & $<0.18$ & $<0.18$ & $<0.22$ & $<0.22$ & $<0.24$ & $<0.20$ \\
\hline Event 2 & $<0.22$ & $<0.20$ & 2.8 & $<0.16$ & $<0.18$ & $<0.18$ & $<0.22$ & $<0.22$ & $<0.24$ & $<0.20$ \\
\hline Event 3 & $<0.22$ & $<0.20$ & $<0.17$ & $<0.16$ & $<0.18$ & $<0.18$ & $<0.22$ & $<0.22$ & $<0.24$ & $<0.20$ \\
\hline Event 4 & $<0.22$ & $<0.20$ & $<0.17$ & $<0.16$ & $<0.18$ & $<0.18$ & $<0.22$ & $<0.22$ & $<0.24$ & $<0.20$ \\
\hline MRL* & 0.22 & 0.20 & 0.17 & 0.16 & 0.18 & 0.18 & 0.22 & 0.22 & 0.24 & 0.20 \\
\hline
\end{tabular}


Table S43. HAMs detected in samples collected during centralized treatment.

\begin{tabular}{|c|c|c|c|c|}
\hline Sample & TCAM & DCAM & BCAM & DBAM \\
\hline \hline \multicolumn{5}{|c|}{ FO/RO Feed (RO concentrate) } \\
\hline Event 1 & $1.1 \pm 0.1$ & $12.0 \pm 0.5$ & $3.2 \pm 0.1$ & $0.55 \pm 0.03$ \\
Event 2 & $1.3 \pm 0.1$ & $12.4 \pm 2.0$ & $6.2 \pm 0.7$ & $1.2 \pm 0.2$ \\
Event 3 & $0.81 \pm 0.13$ & $17.6 \pm 1.7$ & $6.0 \pm 1.1$ & $0.66 \pm 0.14$ \\
Event 4 & $0.45 \pm 0.01$ & $13.1 \pm 0.3$ & $3.3 \pm 0.1$ & $0.27 \pm 0.01$ \\
\hline \multicolumn{5}{|c|}{ FO/RO Permeate } \\
\hline Event 1 & $<0.24$ & $0.30 \pm 0.01$ & $<0.17$ & $<0.22$ \\
Event 2 & $<0.24$ & $0.55 \pm 0.09$ & $0.19 \pm 0.01$ & $<0.22$ \\
Event 3 & $<0.24$ & $0.83 \pm 0.07$ & $<0.17$ & $<0.22$ \\
Event 4 & $<0.24$ & $0.79 \pm 0.15$ & $0.17 \pm 0.01$ & $<0.22$ \\
\hline \multicolumn{5}{|c|}{ RO Feed } \\
\hline Event 1 & N.M. & N.M. & $N . M$. & $N . M$. \\
Event 2 & 0.24 & 2.1 & 1.3 & 0.22 \\
Event 3 & $<0.24$ & 2.4 & 0.59 & $<0.22$ \\
Event 4 & $<0.24$ & 1.8 & 0.35 & $<0.22$ \\
\hline \multicolumn{5}{|c|}{ RO Permeate } \\
\hline Event 1 & $<0.24$ & 0.31 & $<0.17$ & $<0.22$ \\
Event 2 & $<0.24$ & 0.33 & $<0.17$ & $<0.22$ \\
Event 3 & $<0.24$ & 0.61 & $<0.17$ & $<0.22$ \\
Event 4 & $<0.24$ & $<0.14$ & $<0.17$ & $<0.22$ \\
\hline \hline MRL* & 0.24 & 0.14 & 0.17 & 0.22 \\
\hline
\end{tabular}


Table S44. HAMs detected after chloramine treatment in samples collected during centralized treatment.

\begin{tabular}{|c|c|c|c|c|}
\hline Sample & TCAM & DCAM & BCAM & DBAM \\
\hline \hline \multicolumn{5}{|c|}{ Chloramines: FO/RO Feed (RO concentrate) } \\
\hline Event 1 & $1.1 \pm 0.1$ & $22.1 \pm 3.9$ & $11.4 \pm 0.6$ & $2.5 \pm 0.1$ \\
Event 2 & $0.59 \pm 0.33$ & $17.3 \pm 0.7$ & $10.0 \pm 2.5$ & $2.3 \pm 1.4$ \\
Event 3 & $1.5 \pm 0.1$ & $44.0 \pm 1.7$ & $19.1 \pm 1.7$ & $3.5 \pm 0.7$ \\
Event 4 & $0.71 \pm 0.01$ & $47.2 \pm 4.1$ & $18.4 \pm 1.8$ & $3.3 \pm 0.2$ \\
\hline \multicolumn{5}{|c|}{ Chloramines: FO/RO Permeate } \\
\hline Event 1 & $<0.24$ & $0.38 \pm 0.05$ & $<0.17$ & $<0.22$ \\
Event 2 & $<0.24$ & $1.7 \pm 0.1$ & $0.65 \pm 0.02$ & $<0.22$ \\
Event 3 & $<0.24$ & $0.91 \pm 0.08$ & $0.31 \pm 0.15$ & $<0.22$ \\
Event 4 & $<0.24$ & $1.3 \pm 0.2$ & $0.27 \pm 0.05$ & $<0.22$ \\
\hline \multicolumn{5}{|c|}{ Chloramines: RO Feed } \\
\hline Event 1 & N.M. & N.M. & N.M. & $N . M$. \\
Event 2 & 0.40 & 8.0 & 11.6 & 6.6 \\
Event 3 & 0.34 & 7.9 & 5.5 & 1.9 \\
Event 4 & 0.31 & 11.5 & 8.9 & 3.6 \\
\hline \multicolumn{5}{|c|}{ Chloramines: RO Permeate } \\
\hline Event 1 & $<0.24$ & 0.52 & 0.17 & $<0.22$ \\
Event 2 & $<0.24$ & 0.89 & 0.42 & $<0.22$ \\
Event 3 & $<0.24$ & 0.61 & $<0.17$ & $<0.22$ \\
Event 4 & $<0.24$ & 0.31 & $<0.17$ & $<0.22$ \\
\hline \hline MRL* & 0.24 & 0.14 & 0.17 & 0.22 \\
\hline
\end{tabular}


Table S45. HALs detected in samples collected during centralized treatment.

\begin{tabular}{|c|c|c|c|c|}
\hline Sample & TCAL & BDCAL & DBCAL & TBAL \\
\hline \hline \multicolumn{5}{|c|}{ FO/RO Feed (RO concentrate) } \\
\hline Event 1 & $8.6 \pm 0.2$ & $4.5 \pm 0.1$ & $0.20 \pm 0.01$ & $<0.33$ \\
Event 2 & $8.3 \pm 0.7$ & $1.2 \pm 0.3$ & $<0.20$ & $<0.33$ \\
Event 3 & $9.2 \pm 0.5$ & $11.5 \pm 1.6$ & $24.1 \pm 2.0$ & $<0.33$ \\
Event 4 & $6.3 \pm 0.1$ & $7.5 \pm 0.4$ & $3.2 \pm 0.4$ & $<0.33$ \\
\hline \multicolumn{5}{|c|}{ FO/RO Permeate } \\
\hline Event 1 & $<0.15$ & $<0.14$ & $<0.20$ & $<0.33$ \\
Event 2 & $<0.15$ & $<0.14$ & $<0.20$ & $<0.33$ \\
Event 3 & $<0.15$ & $<0.14$ & $<0.20$ & $<0.33$ \\
Event 4 & $<0.15$ & $<0.14$ & $<0.20$ & $<0.33$ \\
\hline \multicolumn{5}{|c|}{ RO Feed } \\
\hline Event 1 & $N . M$. & $N . M$. & $N . M$. & $N . M$. \\
Event 2 & 1.3 & 0.91 & $<0.20$ & $<0.33$ \\
Event 3 & 1.2 & 1.2 & 0.44 & $<0.33$ \\
Event 4 & 1.0 & 0.95 & 0.41 & $<0.33$ \\
\hline \multicolumn{5}{|c|}{ RO Permeate } \\
\hline Event 1 & $<0.15$ & $<0.14$ & $<0.20$ & $<0.33$ \\
Event 2 & $<0.15$ & $<0.14$ & $<0.20$ & $<0.33$ \\
Event 3 & $<0.15$ & $<0.14$ & $<0.20$ & $<0.33$ \\
Event 4 & $<0.15$ & $<0.14$ & $<0.20$ & $<0.33$ \\
\hline \hline MRL* & 0.15 & 0.14 & 0.20 & 0.33 \\
\hline
\end{tabular}


Table S46. HALs detected after chloramine treatment in samples collected during centralized treatment.

\begin{tabular}{|c|c|c|c|c|}
\hline Sample & TCAL & BDCAL & DBCAL & TBAL \\
\hline \hline \multicolumn{5}{|c|}{ Chloramines: FO/RO Feed (RO concentrate) } \\
\hline Event 1 & $8.5 \pm 0.2$ & $2.4 \pm 1.2$ & $<0.20$ & $<0.33$ \\
Event 2 & $6.6 \pm 0.3$ & $0.16 \pm 0.01$ & $<0.20$ & $<0.33$ \\
Event 3 & $4.1 \pm 0.7$ & $4.0 \pm 0.6$ & $0.73 \pm 0.14$ & $<0.33$ \\
Event 4 & $6.8 \pm 0.1$ & $4.8 \pm 0.1$ & $0.61 \pm 0.04$ & $<0.33$ \\
\hline \multicolumn{5}{|c|}{ Chloramines: FO/RO Permeate } \\
\hline Event 1 & $<0.15$ & $<0.14$ & $<0.20$ & $<0.33$ \\
Event 2 & $0.34 \pm 0.02$ & $<0.14$ & $<0.20$ & $<0.33$ \\
Event 3 & $0.17 \pm 0.02$ & $<0.14$ & $<0.20$ & $<0.33$ \\
Event 4 & $<0.15$ & $0.32 \pm 0.06$ & $<0.20$ & $<0.33$ \\
\hline \multicolumn{5}{|c|}{ Chloramines: RO Feed } \\
\hline Event 1 & N.M. & N.M. & $N . M$. & $N . M$. \\
Event 2 & 1.4 & 1.2 & $<0.20$ & $<0.33$ \\
Event 3 & 1.5 & 1.5 & 0.50 & $<0.33$ \\
Event 4 & 1.4 & 1.5 & 0.65 & $<0.33$ \\
\hline \multicolumn{5}{|c|}{ Chloramines: RO Permeate } \\
\hline Event 1 & $<0.15$ & $<0.14$ & $<0.20$ & $<0.33$ \\
Event 2 & 0.60 & $<0.14$ & $<0.20$ & $<0.33$ \\
Event 3 & $<0.15$ & $<0.14$ & $<0.20$ & $<0.33$ \\
Event 4 & $<0.15$ & $<0.14$ & $<0.20$ & $<0.33$ \\
\hline \hline MRL* & 0.15 & 0.14 & 0.20 & 0.33 \\
\hline
\end{tabular}


Table S47. HANs detected in samples collected during centralized treatment.

\begin{tabular}{|c|c|c|c|c|}
\hline Sample & TCAN & DCAN & BCAN & DBAN \\
\hline \hline \multicolumn{5}{|c|}{ FO/RO Feed (RO concentrate) } \\
\hline Event 1 & $<0.18$ & $6.3 \pm 0.3$ & $<0.16$ & $<0.21$ \\
Event 2 & $<0.18$ & $6.7 \pm 0.4$ & $<0.16$ & $<0.21$ \\
Event 3 & $<0.18$ & $10.5 \pm 1.7$ & $4.2 \pm 1.1$ & $0.78 \pm 0.27$ \\
Event 4 & $<0.18$ & $5.7 \pm 0.1$ & $2.1 \pm 0.5$ & $0.33 \pm 0.09$ \\
\hline \multicolumn{5}{|c|}{ FO/RO Permeate } \\
\hline Event 1 & $<0.18$ & $0.77 \pm 0.02$ & $<0.16$ & $<0.21$ \\
Event 2 & $<0.18$ & $1.8 \pm 0.1$ & $<0.16$ & $<0.21$ \\
Event 3 & $<0.18$ & $1.8 \pm 0.1$ & $0.66 \pm 0.03$ & $0.21 \pm 0.01$ \\
Event 4 & $<0.18$ & $1.1 \pm 0.1$ & $0.45 \pm 0.02$ & $<0.21$ \\
\hline \multicolumn{5}{|c|}{ RO Feed } \\
\hline Event 1 & N.M. & N.M. & $N . M$. & $N . M$. \\
Event 2 & $<0.18$ & 1.8 & 0.46 & $<0.21$ \\
Event 3 & $<0.18$ & 1.5 & 0.59 & $<0.21$ \\
Event 4 & $<0.18$ & 1.1 & 0.38 & $<0.21$ \\
\hline \multicolumn{5}{|c|}{ RO Permeate } \\
\hline Event 1 & $<0.18$ & 0.69 & $<0.16$ & $<0.21$ \\
Event 2 & $<0.18$ & 0.75 & 0.44 & $<0.21$ \\
Event 3 & $<0.18$ & 1.1 & 0.52 & $<0.21$ \\
Event 4 & $<0.18$ & 0.44 & 0.18 & $<0.21$ \\
\hline \hline MRL* & 0.18 & 0.14 & 0.16 & 0.21 \\
\hline
\end{tabular}


Table S48. HANs detected after chloramine treatment in samples collected during centralized treatment.

\begin{tabular}{|c|c|c|c|c|}
\hline Sample & TCAN & DCAN & BCAN & DBAN \\
\hline \hline \multicolumn{5}{|c|}{ Chloramines: FO/RO Feed (RO concentrate) } \\
\hline Event 1 & $<0.18$ & $7.7 \pm 1.7$ & $6.4 \pm 1.2$ & $1.7 \pm 0.4$ \\
Event 2 & $<0.18$ & $4.9 \pm 0.1$ & $5.8 \pm 0.1$ & $2.6 \pm 0.1$ \\
Event 3 & $<0.18$ & $12.1 \pm 0.3$ & $6.6 \pm 0.4$ & $1.5 \pm 0.2$ \\
Event 4 & $<0.18$ & $11.8 \pm 0.1$ & $5.8 \pm 0.1$ & $1.9 \pm 0.1$ \\
\hline \multicolumn{5}{|c|}{ Chloramines: FO/RO Permeate } \\
\hline Event 1 & $<0.18$ & $0.70 \pm 0.11$ & $0.24 \pm 0.01$ & $<0.21$ \\
Event 2 & $<0.18$ & $2.4 \pm 0.2$ & $0.45 \pm 0.03$ & $<0.21$ \\
Event 3 & $<0.18$ & $2.3 \pm 0.4$ & $0.81 \pm 0.04$ & $0.51 \pm 0.03$ \\
Event 4 & $<0.18$ & $2.4 \pm 0.1$ & $0.60 \pm 0.03$ & $0.52 \pm 0.03$ \\
\hline \multicolumn{5}{|c|}{ Chloramines: RO Feed } \\
\hline Event 1 & N.M. & N.M. & N.M. & $N . M$. \\
Event 2 & $<0.18$ & 3.2 & 4.1 & 2.6 \\
Event 3 & $<0.18$ & 3.5 & 2.6 & 0.84 \\
Event 4 & $<0.18$ & 4.5 & 2.7 & 1.4 \\
\hline \multicolumn{5}{|c|}{ Chloramines: RO Permeate } \\
\hline Event 1 & $<0.18$ & 0.87 & 0.29 & $<0.21$ \\
Event 2 & $<0.18$ & 0.69 & 0.52 & $<0.21$ \\
Event 3 & $<0.18$ & 0.77 & 0.43 & $<0.21$ \\
Event 4 & $<0.18$ & 1.7 & 0.21 & 0.31 \\
\hline \hline MRL* & 0.18 & 0.14 & 0.16 & 0.21 \\
\hline
\end{tabular}


Table S49. HKs and HNMs detected in samples collected during centralized treatment.

\begin{tabular}{|c|c|c|c|}
\hline Sample & $1,1,1-$ TCP & $1,1-D C P$ & TCNM \\
\hline \hline \multicolumn{4}{|c|}{ FO/RO Feed (RO concentrate) } \\
\hline Event 1 & $6.4 \pm 0.2$ & $10.8 \pm 0.2$ & $<0.16$ \\
Event 2 & $<0.18$ & $8.3 \pm 0.7$ & $<0.16$ \\
Event 3 & $5.9 \pm 0.3$ & $8.1 \pm 0.6$ & $0.76 \pm 0.31$ \\
Event 4 & $5.1 \pm 0.1$ & $6.2 \pm 0.2$ & $0.33 \pm 0.12$ \\
\hline \multicolumn{4}{|c|}{ FO/RO Permeate } \\
\hline Event 1 & $0.24 \pm 0.01$ & $0.80 \pm 0.01$ & $<0.16$ \\
Event 2 & $<0.18$ & $0.82 \pm 0.21$ & $<0.16$ \\
Event 3 & $<0.18$ & $0.22 \pm 0.01$ & $<0.16$ \\
Event 4 & $<0.18$ & $0.26 \pm 0.04$ & $<0.16$ \\
\hline \multicolumn{4}{|c|}{ RO Feed } \\
\hline Event 1 & N.M. & N.M. & $N . M$. \\
Event 2 & $<0.18$ & 1.2 & $<0.16$ \\
Event 3 & 0.90 & 1.2 & $<0.16$ \\
Event 4 & 0.68 & 0.94 & $<0.16$ \\
\hline \multicolumn{4}{|c|}{ RO Permeate } \\
\hline Event 1 & 0.24 & 0.91 & $<0.16$ \\
Event 2 & $<0.18$ & 0.30 & $<0.16$ \\
Event 3 & $<0.18$ & 0.75 & $<0.16$ \\
Event 4 & $<0.18$ & $<0.22$ & $<0.16$ \\
\hline \hline MRL* & 0.18 & 0.22 & 0.16 \\
\hline
\end{tabular}


Table S50. HKs and HNMs detected after chloramine treatment in samples collected during centralized treatment.

\begin{tabular}{|c|c|c|c|}
\hline Sample & $1,1,1-\mathrm{TCP}$ & $1,1-\mathrm{DCP}$ & TCNM \\
\hline \hline Chloramines: FO/RO Feed (RO concentrate) \\
\hline Event 1 & $0.35 \pm 0.14$ & $12.0 \pm 0.9$ & $8.6 \pm 1.7$ \\
Event 2 & $<0.18$ & $6.2 \pm 0.1$ & $1.5 \pm 0.1$ \\
Event 3 & $1.0 \pm 0.1$ & $10.9 \pm 0.2$ & $2.5 \pm 0.6$ \\
Event 4 & $0.82 \pm 0.04$ & $11.5 \pm 0.1$ & $2.1 \pm 0.1$ \\
\hline \multicolumn{4}{|c|}{ Chloramines: FO/RO Permeate } \\
\hline Event 1 & $0.29 \pm 0.08$ & $1.6 \pm 0.1$ & $0.32 \pm 0.05$ \\
Event 2 & $<0.18$ & $1.4 \pm 0.1$ & $0.26 \pm 0.01$ \\
Event 3 & $<0.18$ & $1.7 \pm 0.1$ & $<0.16$ \\
Event 4 & $<0.18$ & $2.8 \pm 0.3$ & $<0.16$ \\
\hline \multicolumn{4}{|c|}{ Chloramines: RO Feed } \\
\hline Event 1 & $N . M$. & $N . M$. & $N . M$. \\
Event 2 & $<0.18$ & 1.4 & 2.2 \\
Event 3 & 0.50 & 2.0 & 1.4 \\
Event 4 & 0.53 & 4.7 & 2.2 \\
\hline \multicolumn{4}{|c|}{ Chloramines: RO Permeate } \\
\hline Event 1 & 0.25 & 1.2 & 0.91 \\
Event 2 & $<0.18$ & 0.29 & $<0.16$ \\
Event 3 & 0.61 & 0.56 & $<0.16$ \\
Event 4 & $<0.18$ & 2.7 & $<0.16$ \\
\hline \hline MRL & 0.18 & 0.22 & 0.16 \\
\hline
\end{tabular}


Table S51. I-THMs detected in samples collected during centralized treatment.

\begin{tabular}{|c|c|c|c|c|c|c|}
\hline Sample & DCIM & BCIM & DBIM & CDIM & BDIM & TIM \\
\hline \hline \multicolumn{7}{|c|}{ FO/RO Feed (RO concentrate) } \\
\hline Event 1 & $<0.20$ & $<0.21$ & $<0.17$ & $<0.21$ & $<0.18$ & $<0.20$ \\
Event 2 & $0.20 \pm 0.01$ & $<0.21$ & $<0.17$ & $<0.21$ & $<0.18$ & $<0.20$ \\
Event 3 & $1.2 \pm 0.2$ & $0.85 \pm 0.04$ & $0.31 \pm 0.11$ & $<0.21$ & $<0.18$ & $<0.20$ \\
Event 4 & $1.2 \pm 0.1$ & $0.37 \pm 0.20$ & $0.32 \pm 0.07$ & $<0.21$ & $<0.18$ & $<0.20$ \\
\hline \multicolumn{7}{|c|}{ FO/RO Permeate } \\
\hline Event 1 & $<0.20$ & $<0.21$ & $<0.17$ & $<0.21$ & $<0.18$ & $<0.20$ \\
Event 2 & $<0.20$ & $<0.21$ & $<0.17$ & $<0.21$ & $<0.18$ & $<0.20$ \\
Event 3 & $<0.20$ & $<0.21$ & $<0.17$ & $<0.21$ & $<0.18$ & $<0.20$ \\
Event 4 & $0.29 \pm 0.04$ & $<0.21$ & $<0.17$ & $<0.21$ & $<0.18$ & $<0.20$ \\
\hline \multicolumn{7}{|c|}{ RO Feed } \\
\hline Event 1 & $N . M$. & $N . M$. & $N . M$. & $N . M$. & $N . M$. & $N . M$. \\
Event 2 & 0.29 & $<0.21$ & $<0.17$ & $<0.21$ & $<0.18$ & $<0.20$ \\
Event 3 & 0.24 & $<0.21$ & $<0.17$ & $<0.21$ & $<0.18$ & $<0.20$ \\
Event 4 & 0.25 & $<0.21$ & $<0.17$ & $<0.21$ & $<0.18$ & $<0.20$ \\
\hline \multicolumn{7}{|c|}{ RO Permeate } \\
\hline Event 1 & $<0.20$ & $<0.21$ & $<0.17$ & $<0.21$ & $<0.18$ & $<0.20$ \\
Event 2 & 0.20 & $<0.21$ & $<0.17$ & $<0.21$ & $<0.18$ & $<0.20$ \\
Event 3 & $<0.20$ & $<0.21$ & $<0.17$ & $<0.21$ & $<0.18$ & $<0.20$ \\
Event 4 & $<0.20$ & $<0.21$ & $<0.17$ & $<0.21$ & $<0.18$ & $<0.20$ \\
\hline \hline MRL* & 0.20 & 0.21 & 0.17 & 0.21 & 0.18 & 0.20 \\
\hline
\end{tabular}


Table S52. I-THMs detected after chloramine treatment in samples collected during centralized treatment.

\begin{tabular}{|c|c|c|c|c|c|c|}
\hline Sample & DCIM & BCIM & DBIM & CDIM & BDIM & TIM \\
\hline \hline \multicolumn{7}{|c|}{ Chloramines: FO/RO Feed (RO concentrate) } \\
\hline Event 1 & $1.6 \pm 0.1$ & $0.59 \pm 0.06$ & $0.23 \pm 0.01$ & $<0.21$ & $0.18 \pm 0.01$ & $<0.20$ \\
Event 2 & $1.7 \pm 0.1$ & $0.63 \pm 0.01$ & $0.29 \pm 0.01$ & $<0.21$ & $<0.18$ & $<0.20$ \\
Event 3 & $1.2 \pm 0.1$ & $0.62 \pm 0.03$ & $0.32 \pm 0.02$ & $<0.21$ & $<0.18$ & $<0.20$ \\
Event 4 & $1.7 \pm 0.1$ & $0.91 \pm 0.02$ & $0.38 \pm 0.02$ & $<0.21$ & $<0.18$ & $<0.20$ \\
\hline \multicolumn{7}{|c|}{ Chloramines: FO/RO Permeate } \\
\hline Event 1 & $<0.20$ & $<0.21$ & $<0.17$ & $<0.21$ & $<0.18$ & $<0.20$ \\
Event 2 & $<0.20$ & $<0.21$ & $<0.17$ & $<0.21$ & $<0.18$ & $<0.20$ \\
Event 3 & $<0.20$ & $<0.21$ & $<0.17$ & $<0.21$ & $<0.18$ & $<0.20$ \\
Event 4 & $<0.20$ & $<0.21$ & $<0.17$ & $<0.21$ & $<0.18$ & $<0.20$ \\
\hline \multicolumn{7}{|c|}{ Chloramines: RO Feed } \\
\hline Event 1 & $N . M$. & $N . M$. & $N . M$. & $N . M$. & $N . M$. & $N . M$. \\
Event 2 & 0.76 & 0.70 & 0.47 & $<0.21$ & 0.25 & $<0.20$ \\
Event 3 & 0.45 & 0.51 & 0.25 & $<0.21$ & $<0.18$ & $<0.20$ \\
Event 4 & 0.79 & 0.97 & 0.62 & 0.21 & 0.35 & $<0.20$ \\
\hline \multicolumn{7}{|c|}{ Chloramines: RO Permeate } \\
\hline Event 1 & $<0.20$ & $<0.21$ & $<0.17$ & $<0.21$ & $<0.18$ & $<0.20$ \\
Event 2 & $<0.20$ & $<0.21$ & $<0.17$ & $<0.21$ & $<0.18$ & $<0.20$ \\
Event 3 & $<0.20$ & $<0.21$ & $<0.17$ & $<0.21$ & $<0.18$ & $<0.20$ \\
Event 4 & $<0.20$ & $<0.21$ & $<0.17$ & $<0.21$ & $<0.18$ & $<0.20$ \\
\hline \hline MRL* & 0.20 & 0.21 & 0.17 & 0.21 & 0.18 & 0.20 \\
\hline
\end{tabular}


Table S53. $N$-nitrosamines detected in samples collected during centralized treatment.

\begin{tabular}{|c|c|c|c|c|c|c|c|c|}
\hline Sample & NDMA & NDEA & NMOR & NPIP & NDBA & NDPA & NPYR & NMEA \\
\hline \hline \multicolumn{7}{|c|}{ FO/RO Feed (RO concentrate) } \\
\hline Event 1 & $148.9 \pm 0.8$ & $127.1 \pm 1.0$ & $114.1 \pm 1.0$ & $<2.3$ & $26 \pm 0.4$ & $<3.0$ & $144.5 \pm 2.2$ & $<2.1$ \\
Event 2 & $59.6 \pm 1.3$ & $3.8 \pm 0.3$ & $88.9 \pm 0.2$ & $<2.3$ & $<2.3$ & $<3.0$ & $<3.0$ & $<2.1$ \\
Event 3 & $130.9 \pm 13.7$ & $<3.0$ & $86.6 \pm 3.3$ & $<2.3$ & $5.8 \pm 0.4$ & $<3.0$ & $34.7 \pm 11.8$ & $<2.1$ \\
Event 4 & $235.1 \pm 14.9$ & $9.7 \pm 3.1$ & $160.4 \pm 19.0$ & $<2.3$ & $30.2 \pm 13.2$ & $<3.0$ & $37.9 \pm 1.2$ & $<2.1$ \\
\hline \multicolumn{7}{|c|}{ FO/RO Permeate } \\
\hline Event 1 & $21.7 \pm 1.1$ & $<3.0$ & $<3.0$ & $<2.3$ & $<2.3$ & $<3.0$ & $55.9 \pm 3.8$ & $<2.1$ \\
Event 2 & $24.5 \pm 1.3$ & $<3.0$ & $<3.0$ & $<2.3$ & $<2.3$ & $<3.0$ & $<3.0$ & $<2.1$ \\
Event 3 & $25.9 \pm 2.6$ & $<3.0$ & $<3.0$ & $<2.3$ & $<2.3$ & $<3.0$ & $<3.0$ & $<2.1$ \\
Event 4 & $44.9 \pm 8.8$ & $<3.0$ & $<3.0$ & $<2.3$ & $<2.3$ & $<3.0$ & $<3.0$ & $<2.1$ \\
\hline \multicolumn{7}{|c|}{ RO Feed } \\
\hline Event 1 & $N . M$. & $N . M$. & $N . M$. & $N . M$. & $N . M$. & $N . M$. & $N . M$. & $N . M$. \\
Event 2 & 16.7 & $<3.0$ & 16.9 & $<2.3$ & $<2.3$ & $<3.0$ & $<3.0$ & $<2.1$ \\
Event 3 & 28.3 & $<3.0$ & 21.7 & $<2.3$ & $<2.3$ & $<3.0$ & $<3.0$ & $<2.1$ \\
Event 4 & 38.1 & $<3.0$ & 19.7 & $<2.3$ & $<2.3$ & $<3.0$ & $<3.0$ & $<2.1$ \\
\hline \multicolumn{7}{|c|}{ RO Permeate } \\
\hline Event 1 & 20.6 & $<3.0$ & $<3.0$ & $<2.3$ & $<2.3$ & $<3.0$ & 59.7 & $<2.1$ \\
Event 2 & 10.6 & $<3.0$ & $<3.0$ & $<2.3$ & $<2.3$ & $<3.0$ & $<3.0$ & $<2.1$ \\
Event 3 & 11.1 & $<3.0$ & $<3.0$ & $<2.3$ & $<2.3$ & $<3.0$ & $<3.0$ & $<2.1$ \\
Event 4 & 16.2 & $<3.0$ & $<3.0$ & $<2.3$ & $<2.3$ & $<3.0$ & $<3.0$ & $<2.1$ \\
\hline \hline MRL & 2.0 & 3.0 & 3.0 & 2.3 & 2.3 & 3.0 & 3.0 & 2.1 \\
\hline
\end{tabular}


Table S54. $N$-nitrosamines detected after chloramine treatment in samples collected during centralized treatment.

\begin{tabular}{|c|c|c|c|c|c|c|c|c|}
\hline Sample & NDMA & NDEA & NMOR & NPIP & NDBA & NDPA & NPYR & NMEA \\
\hline \hline \multicolumn{8}{|c|}{ Chloramines: FO/RO Feed (RO concentrate) } \\
\hline Event 1 & $207.4 \pm 3.6$ & $134.5 \pm 9.0$ & $112.1 \pm 5.1$ & $<2.3$ & $32.4 \pm 2.2$ & $<3.0$ & $163 / 8 \pm 12.5$ & $<2.1$ \\
Event 2 & $118.3 \pm 4.4$ & $34.6 \pm 1.4$ & $80.9 \pm 1.0$ & $<2.3$ & $8.5 \pm 0.1$ & $<3.0$ & $<3.0$ & $<2.1$ \\
Event 3 & $142.2 \pm 14.7$ & $<3.0$ & $109.6 \pm 8.9$ & $<2.3$ & $7.2 \pm 2.1$ & $<3.0$ & $81.5 \pm 1.8$ & $<2.1$ \\
Event 4 & $205.0 \pm 20.6$ & $8.2 \pm 0.1$ & $112.6 \pm 17.9$ & $<2.3$ & $34.0 \pm 7.1$ & $<3.0$ & $76.4 \pm 1.8$ & $<2.1$ \\
\hline \multicolumn{7}{|c|}{ Chloramines: FO/RO Permeate } \\
\hline Event 1 & $29.3 \pm 0.6$ & $<3.0$ & $<3.0$ & $<2.3$ & $<2.3$ & $<3.0$ & $70.3 \pm 12.9$ & $<2.1$ \\
Event 2 & $35.4 \pm 3.5$ & $<3.0$ & $<3.0$ & $<2.3$ & $<2.3$ & $<3.0$ & $<3.0$ & $<2.1$ \\
Event 3 & $32.1 \pm 4.0$ & $<3.0$ & $<3.0$ & $<2.3$ & $<2.3$ & $<3.0$ & $<3.0$ & $<2.1$ \\
Event 4 & $54.6 \pm 5.2$ & $<3.0$ & $<3.0$ & $<2.3$ & $<2.3$ & $<3.0$ & $<3.0$ & $<2.1$ \\
\hline \multicolumn{7}{|c|}{ Chloramines: RO Feed } \\
\hline Event 1 & N.M. & N.M. & N.M. & N.M. & N.M. & $N . M$. & $N . M$. & $N . M$. \\
Event 2 & 282.3 & $<3.0$ & 17.0 & $<2.3$ & $<2.3$ & $<3.0$ & $<3.0$ & $<2.1$ \\
Event 3 & 383.8 & $<3.0$ & 8.1 & $<2.3$ & $<2.3$ & $<3.0$ & 19.5 & $<2.1$ \\
Event 4 & 388.3 & $<3.0$ & 17.4 & $<2.3$ & $<2.3$ & $<3.0$ & 27.3 & $<2.1$ \\
\hline \multicolumn{7}{|c|}{ Chloramines: RO Permeate } \\
\hline Event 1 & 16.3 & $<3.0$ & $<3.0$ & $<2.3$ & $<2.3$ & $<3.0$ & 42.4 & $<2.1$ \\
Event 2 & 16.6 & $<3.0$ & $<3.0$ & $<2.3$ & $<2.3$ & $<3.0$ & $<3.0$ & $<2.1$ \\
Event 3 & 15.1 & $<3.0$ & $<3.0$ & $<2.3$ & $<2.3$ & $<3.0$ & $<3.0$ & $<2.1$ \\
Event 4 & 15.7 & $<3.0$ & $<3.0$ & $<2.3$ & $<2.3$ & $<3.0$ & $<3.0$ & $<2.1$ \\
\hline \hline MRL & 2.0 & 3.0 & 3.0 & 2.3 & 2.3 & 3.0 & 3.0 & 2.1 \\
\hline
\end{tabular}




\section{References.}

Chuang, Y.H., Mitch W.A. The effect of ozonation and biological activated carbon treatment of wastewater effluents on formation of $N$-nitrosamines and halogenated disinfection byproducts. Environ. Sci. Technol. 2017, 51, 2329-2338.

Ferrer, I., Zweigenbaum, J.A., Thurman, E. M., Analytical methodologies for the detection of sucralose in water. Anal. Chem. 2013, 85, 9581-9587.

Jasper, J.T., Sedlak, D.L. Phototransformation of wastewater-derived trace organic contaminants in open-water unit process treatment wetlands. Environ. Sci. Technol. 2013, 47, 10781-10790.

McCurry, D.L., Bear, S.E., Bae, J., Sedlak D.L., McCarty P. L., Mitch W.A. Superior removal of disinfection byproduct precursors and pharmaceuticals from wastewater in a staged anaerobic fluidized membrane bioreactor compared to activated sludge. Environ. Sci. Technol. Lett. 2014, 1, 459-464.

Royal Society of Chemistry, 2019. Chemspider Chemical Structure and Property Database. https://www.chemspider.com/FullSearch.aspx (retrieved October 22, 2019).

Wagner, E.D., Plewa, M.J., CHO cell cytotoxicity and genotoxicity analyses of disinfection byproducts: an updated review. J. Environ. Sci. 2017, 58, 64-76. 